\title{
Neural population dynamics and frontal-parietal circuit for context-dependent sensorimotor computations
}

\author{
Dissertation \\ for the award of the degree \\ “Doctor rerum naturalium” \\ of the Georg-August-Universität Göttingen
}

within the doctoral program Theoretical and Computational Neuroscience

$$
\text { of the Göttingen Graduate Center for }
$$

Neurosciences, Biophysics, and Molecular Biosciences (GGNB)

submitted by

\section{Hao Guo}

from Jinan, China

Göttingen 2019 


\section{Doctoral Thesis Committee:}

Prof. Dr. Alexander Gail (First Referee, Supervisor)

Sensorimotor Group

German Primate Center

Prof. Dr. med. Hansjörg Scherberger (Second Referee)

Neurobiology Laboratory

German Primate Center

Prof. Dr. Fred Wolf

Theoretical Neurophysics Group

Max Planck Institute for Dynamics and Self-Organization

\section{External Examiners:}

Prof. Dr. Tobias Moser

University Medical Center Göttingen

Institute for Auditory Neuroscience \& InnerEarLab

Prof. Dr. Jochen Staiger

University Medical Center Göttingen

Center of Anatomy, Dept. of Neuroanatomy

Prof. Dr. Tim Gollisch

University Medical Center Göttingen

Sensory Processing in the Retina

Date of oral examination: May 23 ${ }^{\text {rd }}, 2019$ 
I hereby declare that this thesis has been written independently and with no other aids and sources other than quoted.

Göttingen, April 5 ${ }^{\text {th }}, 2019$

Hao Guo 


\section{Acknowledgements}

I am grateful to all the people who supported me and made this dissertation work possible. First of all, I would like to thank Prof. Dr. Alexander Gail for giving me this opportunity to work on an exciting project in his lab. He provided me constant support, creative ideas, constructive criticism and continuous encouragement in the past years. I also want to thank my thesis committee members, Prof. Dr. med. Hansjörg Scherberger and Prof. Dr. Fred Wolf, for their helpful advice and discussions during each stage of the thesis.

Many people in the lab helped me when I was facing technical hurdles, and provided a contribution to the present work. Special thanks go to Sina Plümer, Luisa Klotz, Leonore Burchardt and Dirk Prüsse for their guidance and helping me with handling and training monkeys, Klaus Heisig for the mechanical and technical issues, Ralf Brockhausen and Matthis Drolet for the IT and software issues. I thank Beatrix Glaser for all kinds of administrative support.

I am lucky to work with the best colleagues. I would like to thank all the former and present members in the Sensorimotor Group for their inspiring discussions and valuable feedbacks. I am especially thankful to Pierre Morel who supported me in data analysis and scientific programming, and Michal Fortuna who helped with his suggestions regarding optogenetic topics in my work.

I am thankful to all the former and present colleagues in the Cognitive Neuroscience Laboratory and Decision and Awareness Group for making a friendly lab atmosphere and all scientific feedbacks regarding my project.

I would like to thank all my friends in Göttingen. I thank them for making life so enjoyable and colorful.

Last but not least, a special thank is dedicated to my family, my parents for always supporting me, encouraging me and believing in me no matter what I chose to do. 


\section{Contents}

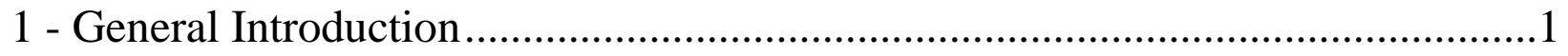

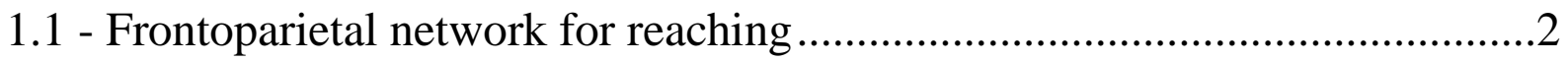

1.2 - Motor goal tuning: representational perspective ….......................................5

1.3 - Inter-areal coordination in the frontoparietal network ................................8

1.4 - Neural computation: dynamical system perspective ..................................12

1.4.1 - Role of preparatory activity: setting the initial state ...........................14

1.4.2 - Neural manifold alignment ..............................................................15

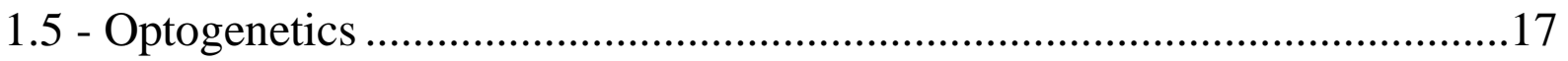

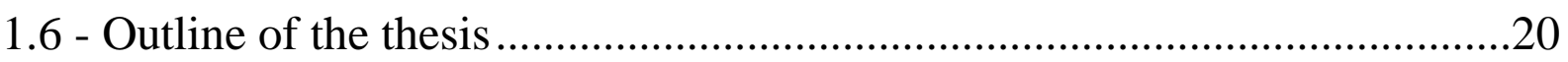

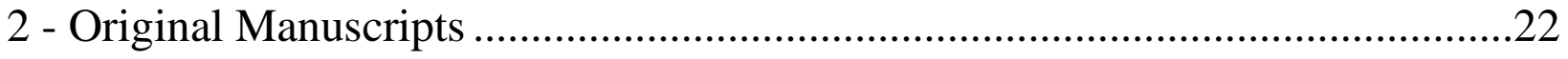

2.1 - Shared preparatory tuning representation but distinct peri-movement neuronal dynamics in monkey frontal and parietal reach areas..........................23

2.2 - Reconfiguration of population dynamics for context-dependent sensorimotor transformations..........................................................................66

2.3 - Optogenetic inhibition of premotor-to-parietal projections in rhesus monkeys reveals a causal role in rule-based sensorimotor transformations........99

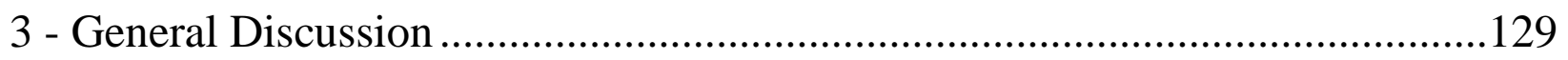

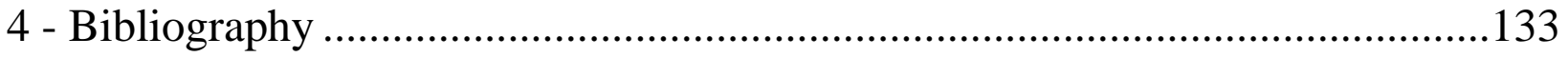




\section{1 - General Introduction}

Movement behavior is how we communicate and interact with the physical world around us. The ability to use fingers, hands and arms in voluntary goal-directed actions enables primates, especially humans exert influences on the surroundings. Planning and control of voluntary goal-directed movements are not as simple as they might appear. It is a distributed task that engages multiple interconnected brain areas in the primate's brain.

The present thesis investigates the computational strategies in primate's brain for goal-directed movement control and the information stream in inter-areal brain circuits during movement preparation. The model system used in this thesis is behaving rhesus monkey. Two approaches were used to study different topics. First, to investigate the underlying neural computations, neural state space methods were applied onto extracellular recording datasets. The datasets were collected from rhesus monkeys while they were performing goal-directed reach movements in distinct behavioral contexts. Second, an optogenetic approach in rhesus monkey was developed and used to investigate the function of the inter-areal information stream. Activities of single neurons in two distinct cortical areas, which are involved in reach planning, were recorded in combination with optogenetic inhibition.

The thesis is organized into three chapters. The first chapter provides a general introduction. The second chapter consists of three scientific manuscripts in preparation, which investigates different aspects of the research topic. The first manuscript investigates the neural computation during planning-to-execution transition and the functional differences between distinct cortical areas. The second manuscript investigates the computational strategies which are exploited by the 
brain for movement preparation and estimates how the computations differ in different behavioral contexts. The third manuscript discusses the results of the optogenetic manipulations performed in one rhesus monkey, and the function of information flows between interconnected brain areas during sensorimotor computations. At the beginning of each manuscript, a brief introduction is provided, and followed by the original manuscript. The third chapter summarizes the results of the presented projects and draws a general conclusion.

\section{1 - Frontoparietal network for reaching}

Goal-directed movement is the result of complex cognitive processes. In our daily life, we always encounter numerous objects out of which we only aim for the selected one. Consider an action of picking a piece of fruit from a bowl on the table in front of us. Even such a simple action involves multiple processing and acting stages. The positions of objects in space relative to my body and hand, the target selection based on their color and appearance, the muscle activation for precise reaching and grasping movement, even the anticipated sensory feedback, must be processed by the brain.

Goal-directed movements are mediated by specialized areas distributed across the cerebral cortex in an orderly arrangement, particularly by directed interactions between the frontal lobe and the parietal lobe. In human and non-human primates (NHPs), the parietal cortex locates between the frontal and the occipital lobe. The dorsal portion of the parietal cortex receives inputs from multiple sensory modalities (Colby et al., 1988), and is the central part for integrating sensory and motor information. The posterior parietal cortex (PPC) is involved in action planning (Snyder et al., 1997; Buneo et al., 2002) and contributes to a diverse array 
of goal-directed movements. Various subareas in PPC are characterized by segregated functions (Grefkes and Fink, 2005), such as eye-movement, reaching, and grasping in space. These areas in PPC connect to multiple areas in the frontal lobe with similar functional selectivity (Johnson et al., 1996; Tanne-Gariepy et al., 2002; Caminiti et al., 2017). The functionally defined parietal reach region (PRR) (Andersen and Buneo, 2002; Cohen and Andersen, 2002) in the PPC, and the dorsal premotor cortex (PMd), together form the frontoparietal network for arm reaching (Fig. 1) (Johnson et al., 1996; Cisek and Kalaska, 2010). This frontoparietal reach network is involved in transforming spatial coordinates of the target into a motor-goal representation that can be used by the motor system for reaching.

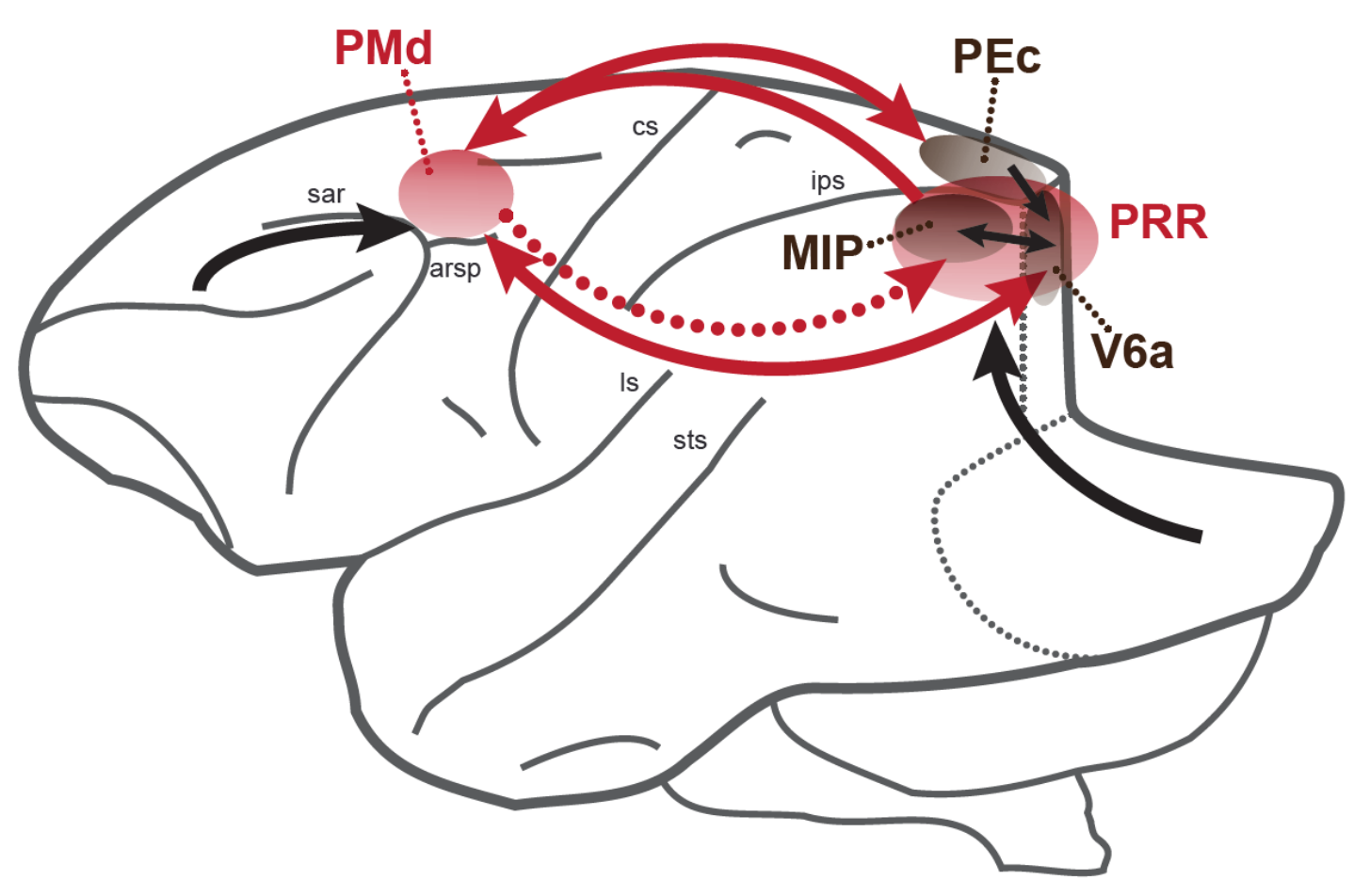

Figure 1: Schematic drawing of the frontoparietal network for reaching. The network includes areas PMd and PRR. Arrows indicate the presumed information stream. 
PRR was initially identified as an area medial and posterior to the lateral intraparietal area (Snyder et al., 1997) thus might have included more than one cortical area. Many subsequent studies which explored the function of the PRR, defined it as an area that anatomically consists of the medial bank of the intraparietal sulcus (MIP), and likely parts of the visual area 6 in the anterior bank of the parietooccipital sulcus (V6a) (Snyder et al., 1997; Batista et al., 1999). The inter-areal pathways linking PRR and other cortical areas have been elucidated by many anterograde and retrograde tracing experiments (Fig. 1). PRR receives its afferent from areas that are directly connected to, or are considered part of, the extrastriate visual cortex (Felleman and Van Essen, 1991). V6a area receives dense projections from PMd in the frontal lobe (Caminiti et al., 1985; Caminiti et al., 1999; Marconi et al., 2001; Gamberini et al., 2009; Bakola et al., 2010) and PEc (Gamberini et al., 2009) in the parietal lobe. Within the PRR region, V6a are mutually interconnected with the neighboring MIP (Gamberini et al., 2009; Passarelli et al., 2011). Although PRR, which is directly connected with the visual cortex, is believed to play an essential role in sensory functions, a number of studies suggested that PRR shows activity related to planning a voluntary reach movement (Snyder et al., 1997; Cui and Andersen, 2007; Scherberger and Andersen, 2007; Cui and Andersen, 2011). The behavioral modulations induced by inactivating PRR in monkey supports the notion that PRR is causally involved in reach movement (Hwang et al., 2012).

PMd is a critical node in the frontal lobe that is intimately involved in voluntary reach movements (Kurata, 1989; Johnson et al., 1996; Cisek and Kalaska, 2010). PMd is located in the dorso-caudal part of Brodmann's area 6, lies anterior to the primary motor cortex (M1), and posterior to the frontal lobe. Anatomical studies have confirmed that PMd receives various inputs from multiple brain areas (Fig. 1), 
such as, various subareas in the prefrontal cortex (Barbas and Pandya, 1987, 1989; Marconi et al., 2001; Petrides and Pandya, 2006; Markov et al., 2014), MIP (Johnson et al., 1996; Petrides and Pandya, 1999; Luppino et al., 2001; Marconi et al., 2001; Tanne-Gariepy et al., 2002; Luppino et al., 2003; Markov et al., 2014), V6a (Matelli et al., 1998; Caminiti et al., 1999; Gamberini et al., 2009) and PEc (Tanne-Gariepy et al., 2002). PMd neurons are characterized by systematically modulating their activity during the reach movement preparation and execution phase (Crammond and Kalaska, 1994; Pesaran et al., 2006; Batista et al., 2007), which provides the explanation from a physiological perspective of the behavioral deficits of PMd lesions in monkeys (Kurata and Hoffman, 1994). A recent study applying reversible inactivation (Ohbayashi et al., 2016) in behaving monkeys emphasized the role of the PMd in internally generated, but not visually guided, sequential reach movements.

Physiological studies have shown that PMd and PRR sharing similar functional properties tend to be preferentially linked through inter-areal connections. The electrophysiological recordings reported in this thesis were conducted in these two motor-related brain areas. The following sections will focus on the neurophysiological properties of PMd and PRR neurons and their coordinated function in reach planning.

\section{2 - Motor goal tuning: representational perspective}

The representational view of single-neuron tuning is firmly rooted in the history of neuroscience (Yuste, 2015) and has been widely used in identifying neurophysiological properties of a given brain area. If the firing rate of a neuron systematically changes when an externally controlled parameter changes, then the 
functional dependency of the firing rate on this parameter could be described as a tuning function, and accordingly, the neuron is called "tuned" for this parameter.

To understand the tuning properties of PMd and PRR neurons in reach movement, tasks with precisely controlled and separated parameters were designed for measuring resulting physical/motor output and accounting for neural activity in terms of tuning for movement parameters. In a so-called centered-out delayed reach task, a flashed visual stimulus on the screen indicates the motor goal, and the movement has to be withheld up to seconds before the actual movement is executed; thus the movement preparation period (and underlying neural process) is disassociated from the movement execution. In a "pro-reach", the subject is instructed to execute a reaching movement towards the visual cue. With the combination of single-unit recording techniques in monkeys, some studies have indicated that neurons in PMd and PRR are spatially tuned for the direction during movement preparation and execution (Crammond and Kalaska, 1994; Snyder et al., 1997). This dependency of the firing rate on the upcoming movement (spatial selectivity) is referred to as motor goal tuning. The neural activity in PMd and PRR is multiphasic as the task proceeds: neuron often exhibits transient activation during the visual cue presentation, followed by the sustained activity during the delay period when the visual stimulus is not available any longer. The sustained activity typically defines the spatially directional selectivity during reach planning.

In centered-out delayed reach task, monkey reaches to the motor goal that is spatially instructed by visual stimulus. Thus, the motor goal tuning defined in this task could be ascribed to visual memory. An "anti-reach" task has been applied to spatially dissociate the motor goal from visual memory by instructing the monkey to reach to a motor goal that is opposite to the appearance of stimulus (Gail and Andersen, 2006; Gail et al., 2009; Westendorff et al., 2010; Klaes et al., 2011). In 
the pro-/anti-reach task, context rules are applied to the visual stimuli that either instruct a reach toward the visual cue (rule pro) or its opposite location (rule anti). If a neuron's response varies with the location of the stimulus regardless of whether the behavioral demand is reaching toward or opposite to it, it would indicate that the neuron encodes visual memory. If, in contrast, a neuron's response only systematically changes its activity in response to the direction of the upcoming reach, the neuron is defined as tuning for the motor goal. Electrophysiological studies in monkeys have demonstrated that sustained neural activities in PMd and PRR are correlated with the intended direction of the reaching movement, rather than the memorized position of the visual cue (Gail and Andersen, 2006; Gail et al., 2009; Westendorff et al., 2010). The same result has also been observed in human posterior parietal cortex while subjects were asked to perform the pro-/anti-reach task (Gertz and Fiehler, 2015).

The neural system computes the position of an object that serves as the target for goal-directed reaching. The resulting changes in the world generate perceptual feedback to the neural system, closing the control loop. A reversing-prism was used in "prism-reach" task to separate the physical movement from its associated visual feedback. Evidence in PRR (Kuang et al., 2016) has proved that action planning is not exclusively a precursor of impending physical movements, as reflected by the predominant physical goal encoding, but also correlates with spatial kinematic parameters of upcoming visual aspects of movement, as reflected by co-existing visual goal encoding in neuronal spiking.

A variety of well-designed tasks have enriched the functional repertoires of PMd and PRR. Both areas are believed to potentially play complementary and differential roles in movement planning (Gail et al., 2009; Westendorff et al., 2010). A recent study investigated putatively different functional roles between 
PMd and PRR based on neural synchronization patterns (Chakrabarti et al., 2014). The unequal local functional organizations observed in PMd and PRR support the idea that these two areas reflect different network architectures to support different functional roles within the frontoparietal reach network. However, whether and how these spatial representations differ across frontal and parietal areas is still elusive. The limitation of the representational view of single-neuron tuning obscures the quantitative comparison between two sensorimotor areas. When seeking representation in a well-designed task, one asks which controlled task parameters are encoded by neuronal spiking. This procedure always involves selecting neurons based on how task-related they appear (significant tuning), thereby biasing recordings and obscuring the actual differences. There are growing bodies of experimental data that are difficult to investigate from a representational framework but become more approachable when dynamical systems concepts are used (Ganguli et al., 2008; Machens et al., 2010). Section 1.4 deals with reach control from the dynamical system perspective.

\section{3 - Inter-areal coordination in the frontoparietal network}

PMd and PRR share basic functional properties and their activities are believed to be coordinated through a reciprocal connection (Johnson et al., 1996). The anatomical architecture of the frontoparietal network imposes constraints on the nature of the coordinate activity underlying goal-directed reach planning. The reach planning computation cannot be regarded as the result of a parallel process performed by respective areas but is the result of a recursive mechanism in which different signals are selected and combined throughout the frontoparietal network and further presented locally. This section describes the inter-areal responses 
within the frontoparietal network during the integration of sensory and contextual information.

In goal-directed reach movement, the same location of a visual stimulus can lead to very different decisions and actions, depending on a specific behavioral context. While sensory stimuli provide information about the location of possible targets and evoke an intention to reach for them, behavioral context directs the incentives and imposes constraints to act in a specific way, thus determines which action is adequate (for instance, to reach toward or to avoid the target). Therefore, motor goal selection requires the integration of sensory and contextual information.

The cognitively controlled motor goal selection has been associated with the frontoparietal network in the cerebral cortex of primates. Given diverse behavioral contexts, neurons respond differently even when the same sensory stimulus is presented. It is known that neurons in the prefrontal cortex are selective for task rules (Asaad et al., 1998; Wallis et al., 2001; Wallis and Miller, 2003) and were shown to represent learned visuomotor associations in different tasks (Asaad et al., 1998; Everling and DeSouza, 2005). In monkeys that have learned to associate arbitrary rules with particular movements, many neurons in the PMd and PRR respond selectively to specific rules (Wallis and Miller, 2003; Gail and Andersen, 2006; Westendorff et al., 2010; Kuang et al., 2016). Within the frontoparietal reach network, the anatomical position of the PMd makes it an ideal candidate as an interface for integrating the sensory information received from the parietal cortex with the contextual information received from the prefrontal cortex (Wallis and Miller, 2003).

Timings within the frontoparietal network provide some insights into which areas may implement contextual tuning and modulation earlier. The relative timing of 
motor goal encoding between PMd and PRR can help parse out the direction of information stream within the frontoparietal reach network. One conventional approach to investigate the issue is to examine inter-areal interactions by recording simultaneously single units and local field potentials from PMd and PRR. Based on the anatomical organization of frontoparietal reach network, one would expect that rule-related activity begins earlier in the frontal lobe areas and passes back to the parietal areas. This hypothesis was tested in instructed and autonomous decisions for selecting spatial locations (Pesaran et al., 2008). When monkeys were engaged in decision-related reach task, spike-field coherence suggested that the PMd to PRR link is activated first, followed by a hand-shake back from PRR to PMd within a few milliseconds. These results also indicated that there might be a subset of cells which engage in mutual communication between PRR and PMd and coordinates the decision process (Pesaran et al., 2008).

A similar observation of PMd responding earlier than PRR has also been found when monkeys were engaged in pro-/anti-reach task (Westendorff et al., 2010). Nearly all spatially tuned neurons in PMd and PRR encode the motor goal and not just the position of the spatial cue, proving that at planning stage the spatial information is already integrated with the contextual information, although the representation of the spatial position of the cue did exist before that. Simultaneous recordings in these two areas showed that neural representations of motor goals appear earlier in PMd than in PRR. It is noteworthy that the timing within this circuit depends on the task contexts: the more natural pro-reach produces approximately simultaneous motor goal representations in PMd and PRR; PMd precedes PRR only in the case of anti-reach when a spatial remapping is required. These motor goal latencies were interpreted as reflecting a dynamic reorganization 
of the network activity in PRR, and the dynamic reorganization of network activity might be contingent on frontoparietal inputs from PMd (Westendorff et al., 2010).

The electrophysiology results showed that the contextual information, which could be an internally represented context (Pesaran et al., 2008) or a learned visuomotor association (Westendorff et al., 2010), is integrated with the spatial information about the target to define the final reach goal. Previous modeling studies indicated that frontoparietal projections might help to sustain motor goal memory (Cisek, 2006) or mediate rule-based visuomotor transformations (Brozovic et al., 2007). Theoretical study (Brozovic et al., 2007) suggested that rule information could originate from the feedback from the motor output layer of the network (top-down) or classical feed-forward projections (bottom-up), although the authors suggested that the former route is more likely to exist.

Evidence presented in neuronal spiking analyses suggested that the PMd and PRR form a hierarchical sensorimotor circuitry that coordinates the decision process and rule-based visuomotor transformations. Notably, the motor goal representations in the PRR during rule-based visuomotor transformations might be the consequence of top-down feedback projection from PMd. While studies of single-cell activity clearly support this hypothesis, a recent study based on Granger-causality measure of intracortical local field potentials argued against the functional interaction within the frontoparietal network (Martinez-Vazquez and Gail, 2018). In the memory-guided rule-based reaches, task conditions were designed to disassociate the motor-goal selection and retrieval of motor-goal memory. Low-frequency PMd activity had a transient Granger-causing effect on PRR specifically during working memory retrieval of spatial motor goals, while no frontoparietal directed interaction was associated with motor-goal selection. 
The question which motivates our research is whether frontoparietal projections from PMd to PRR causally subserve the dynamic visuomotor transformations required in rule-based motor goal planning. In the third research manuscript of this thesis, we tested this hypothesis by using the optogenetic approach.

\section{4 - Neural computation: dynamical system perspective}

The adoption of the representational perspective has biased the research towards particular types of analyses: explain most neural activity in terms of tuning for task-related parameters. Indeed, neurons in the network coordinate together for motor control, rather than function as separated perceptual units that represent arbitrarily defined parameters.

In recent years, the necessity of selecting units based on their tuning properties has been reconsidered. Instead, high-dimensional neural state space is constructed in the sense of dynamical systems across a variety of paradigms (Stopfer et al., 2003; Churchland et al., 2010; Churchland et al., 2012; Harvey et al., 2012). The dynamical system is a physical system defined in state space whose future state is a function of its current state, its external input, and possibly some noise. In a neural state space, each dimension represents the firing rate (FR) of one recorded neuron. The FRs across all neurons at a given time corresponds to a point in neural state space. The FRs trace out a neural trajectory over time (Fig. 2). The observed complexity of the neuronal responses is thus explained in the framework of a highdimensional dynamical process at the level of neural population.

The neural population activity does not explore the full high-dimensional neural state space but can instead be confined to a remarkable low-dimensional subspace, 
which is described by a small number of neural covariation patterns (Cunningham and Yu, 2014; Gallego et al., 2017). These covariation patterns comprise the neural manifold (Fig. 2) within the full space. A theoretical argument described how the concept of a neural manifold arises when studying neural population activity in different tasks, and how the dimensionality of such a manifold is determined by the complexity of the behavioral task (Gao and Ganguli, 2015).

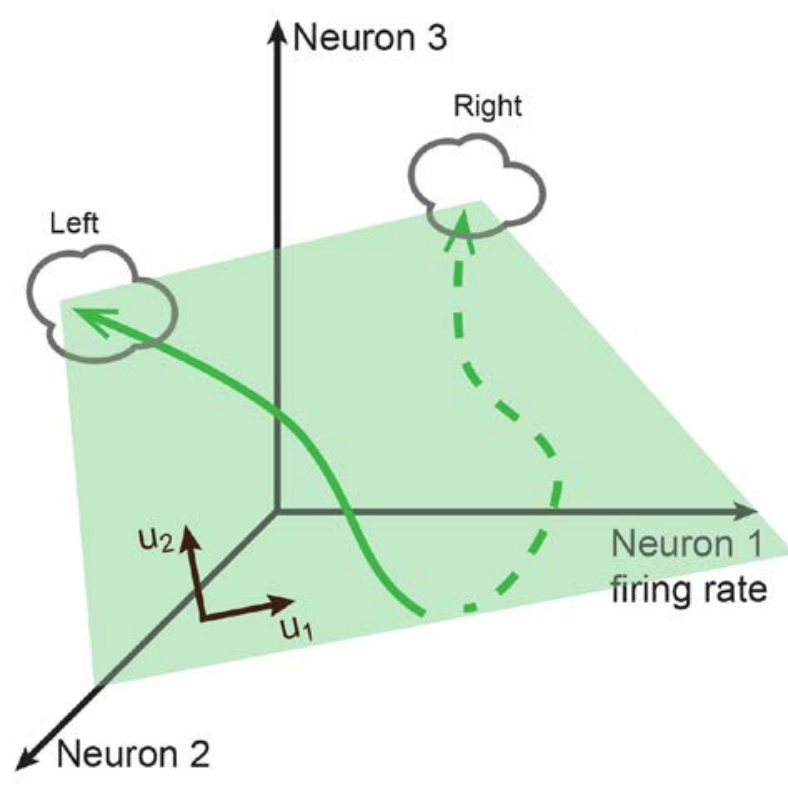

Figure 2: Conceptual illustration of neural state space which is composed of three neurons. The points trace out neural trajectories over time (solid and dashed green lines). The transparent surface represents the 2-dimensional subspace of activity patterns of those three neurons measured during a reaching task, which we refer to as the neural manifold.

Recent advances in dimensionality reduction algorithms have made it possible to examine network-level hypotheses of movement generation, by projecting the responses of many neurons onto a small number of variables that capture the basic covariation patterns present in those responses (Cunningham and $\mathrm{Yu}, 2014$ ). Principal component analysis (PCA) is one of the most widely used dimensionality 
reduction technique for visualizing trial-averaged neuronal spiking data. The goal of PCA is to yield a new coordinate system for a multivariate dataset, such that the first coordinate accounts for as much of the variance (most meaningful basis) in the data as possible, the second coordinate for as much of the remaining variance as possible, and so on. Consider a dataset $X$ consisting of $\mathrm{n}$ points in a D-dimensional space: $x_{1}, x_{2}, \ldots, x_{n} \in \mathbb{R}^{D}$, dimensionality reduction methods map $X$ to $Y$ : $y_{1}, y_{2}, \ldots, y_{n} \in \mathbb{R}^{d}(\mathrm{~d}<<\mathrm{D})$. Given a unit vector $u$, the length of the projection of $x_{i}$ onto $u$ is given by $x_{i}^{T} u$. In PCA, the best direction/subspace for projection lies in the direction of largest variance:

$$
\operatorname{var}(\mathrm{Y})=\frac{1}{n} \sum_{i=1}^{n}\left(x_{i}^{T} u\right)^{2}=\frac{1}{n} \sum_{i=1}^{n} u^{T} x_{i} x_{i}^{T} u=u^{T}\left(\frac{1}{n} \sum_{i=1}^{n} x_{i} x_{i}^{T}\right) u
$$

Here, $\mathrm{C}=\frac{1}{n} \sum_{i=1}^{n} x_{i} x_{i}^{T}$ is the empirical covariance matrix of the data. PCA maximize $u^{T} C u$ by giving the principle eigenvector of $C$. To project the data into a d-dimensional subspace, we choose the top d eigenvectors of $C: u_{1}, u_{2}, \ldots, u_{d}$ to form a new orthogonal basis for representing the data.

The geometric structure of the neural manifold and how the neural population activity temporally evolves within it (the "neural population dynamics”) have been emphasized in many studies recently (see below).

\subsection{1 - Role of preparatory activity: setting the initial state}

Since the dynamical systems framework indicates that current neural population response should evolve predictably in neural state space, one might ask how the population's preparatory state is determined and consequently influences the subsequent neural activity and the movement (Churchland et al., 2010; Churchland 
et al., 2012). Population-level analyses from M1/PMd have yielded several advances in the characterization of preparatory activity, including relating responses during the preparatory period to responses during movement execution (Kaufman et al., 2014; Elsayed et al., 2016), and assessing the necessity of the preparatory state (Ames et al., 2014) by emphasizing that the preparatory dynamical system contains a putative attractor corresponding to the planning state. One viewpoint that has emerged from these studies of population-level preparatory activity is the "initial condition hypothesis" of the motor preparation, which indicates that preparatory activity acts to set an initial condition which leads directly to the subsequent trajectory of a movement-related neural dynamics (Churchland et al., 2010; Afshar et al., 2011; Churchland et al., 2012; Elsayed et al., 2016; Even-Chen et al., 2019).

\subsection{2 - Neural manifold alignment}

Using dimensionality reduction methods, the activity of hundreds of neurons can be represented in a reduced-dimensional neural manifold (i.e., subspace) that reflects the covariance across the neural population (Gallego et al., 2017). It is believed that the underlying network connectivity constrains these possible covariance patterns of population activity (Sadtler et al., 2014; Gallego et al., 2017) and the way neurons co-vary with respect to each other is confined to a lowdimensional manifold spanned by a few independent patterns that are called “neural modes” (Gallego et al., 2017). The neural mode provides the basic building blocks of neural dynamics and can be treated as the signature of a specific neural computation process. If two computations are internally identical, the covariance pattern (neural manifold) is preserved despite the differences between single 
neuron activity patterns. On the contrary, the neural state explores other patterns of neural covariance (different dimensions in state space) when the brain needs to perform a substantially different computation. Figure 2 depicts a simple threeneuron example to illustrate this hypothesis. The manifold alignment that aligns disparate datasets and extracts the common set of features is the important solution for understanding the neural computation and a framework for discovering a unifying representation of multiple datasets.

When behavioral demands differed, multiple computations, thus different manifolds could be implemented in the same neural population. Previous statespace analysis studies have revealed that the underlying computational strategies as the animal's behavioral demand evolved from decision formation to movement execution (Raposo et al., 2014), and from planning to movement (Kaufman et al., 2014; Elsayed et al., 2016). PPC neurons in rodents exhibited different covariance patterns (explored different dimensions) during multi-sensory decision formation and movement (Raposo et al., 2014). A recent study in PMd proved orthogonality between the preparatory and movement subspaces, implying that the neural population activity explored different manifolds during the flexible transitions of two sequential epochs (Elsayed et al., 2016). These findings support the hypothesis that neural population in specific brain area possesses reservoirs of component patterns that can be arbitrarily recruited to perform different computations.

Furthermore, monkeys could learn brain-machine interface (BMI) mappings that lie with the manifold, conforming to existing patterns of neural covariation, but usually could not learn to generate novel neural covariation patterns outside the neural manifold (Sadtler et al., 2014). However, given more time, monkeys could learn outside manifold BMI mappings, and they did so by generating different covariation patterns, outside the neural manifold. This observation addressed 
potential connections between learning and the emergence of neural manifolds and supported the notion that the neural manifold represents a relevant neural computation.

The dynamical systems perspective helps to understand why neural activity evolves the way it does and furthers the understanding of the movement planning, neural control of movement, and motor learning. Although it is an ongoing debate whether the manifold represents a real neurophysiological entity, these studies highlighted the potential of the dynamical system framework, as most of these observations could not have been made by analyzing only single-neuron activity.

\section{5 - Optogenetics}

How do premotor and parietal areas functionally interact in the rule-based visuomotor transformations? This question remains unsatisfactorily addressed because the direct evidence that proves the existence of information streams is currently lacking.

The fundamental principle of directly investigating the role of information streams between interconnected brain areas is straightforward: to perturb neural activity in defined unidirectional projection pathways, while observing the consequences on neuronal and behavioral modulations. Given the transient properties of frontoparietal interaction, the experimental perturbations should be performed with high temporal precision.

The development of optogenetics (Deisseroth, 2015; Grosenick et al., 2015) offers potential tools for achieving this goal. Optogenetics implements perturbations by introducing into neurons light-activated ion channels and pumps that regulate the 
currents on the membrane. These proteins, encoded by microbial opsin genes, allow optical depolarization or hyperpolarization of specific neuron types. The cation channel channelrhodopsin-2 (ChR2, blue light-activated inward cation channel) was the original depolarizing optogenetic tool (Boyden et al., 2005). In order to silence neuronal activity, two fast inhibition rhodopsin classes were initially developed: halorhodopsin (NpHR, yellow light-activated inward chloride pump) (Han and Boyden, 2007; Zhang et al., 2007) and archaerhodopsin (Arch/ArchT, green light-activated outward proton pumps) (Chow et al., 2010; Han et al., 2011). Optogenetics meet the unique challenge in neuroscience because of three essential properties: 1) genetic targetable; 2) millisecond temporal precision; 3) circuit-specific manipulation.

Optogenetics targeting genetically defined cell types is mainly ascribed to the promotor fragments in the viral vector that drives transgene expression. Human synapsin I (hSyn1) and Thy1 (Diester et al., 2011) are both neuron-specific (excluding glia) promoters. The CaMKII $\alpha$ is the excitatory neuron-specific promoter which has been proven successful in targeting excitatory neurons in cortex and hippocampus in rodents and primates (Boyden et al., 2005; Zhang et al., 2007; Lee et al., 2010; Han et al., 2011; Lu et al., 2015). A recent study demonstrated selective optogenetic control of the Purkinje cells in the cerebellum by exploiting L7 promotor (El-Shamayleh et al., 2017). The inhibitory neuronspecific promoter, such as parvalbumin (PV), has not been successfully applied in primates because of the packaging limit of viral vectors. However, due to the development of mDlx enhancer elements, recent research (Dimidschstein et al., 2016) highlighted the potential of targeting and manipulating inhibitory interneurons in non-genetically tractable animal models. Opsins can be readily expressed in neurons by using a variety of transfection techniques 
(viral transfection, electroporation) or using transgenic animals. Delivering an opsin gene by viral vectors remains the best strategy for genetically intractable species such as rhesus monkey (Han et al., 2009; Diester et al., 2011).

Optogenetics enables neural interventions over a broad range of temporal timescales. High temporal precision and reversibility are the primary reasons why optogenetics overcomes traditional methods such as pharmacological and lesionbased interventions, although the latter two are suitable for slow and chronic timescales. Previous studies provided a direct demonstration that ChR2 depolarizes neurons and elicit precisely timed action potentials (Li et al., 2005; Nagel et al., 2005; Gunaydin et al., 2010). NpHR and Arch/ArchT were also proved to meet the requirements of achieving fast kinetics (Gerits and Vanduffel, 2013).

Optogenetics could selectively perturb the activity of neural pathways that connect two brain areas, by delivering light to opsin-expressing axon terminals (anterograde projection targeting). This property is based on the fact that opsins expressed in cell body could be delivered to axon terminals through axonal trafficking, and the opsins expressed at axonal terminals could be activated locally. Illuminating axonal terminals, which express ChR2, causes synaptic release and has been used to map the excitatory inputs onto cortical pyramidal cells (Petreanu et al., 2009) and to reveal synaptic pathways controlling sensation and behavior (Rajasethupathy et al., 2015). A previous study (Mattis et al., 2011) also demonstrated that activation of hyperpolarizing opsins at presynaptic boutons attenuated evoked synaptic transmission. Selective inhibition of projections between brain regions by optogenetics has been proved successful in diverse cognitive and motor tasks (Tye et al., 2011; Jennings et al., 2013; Adhikari et al., 2015; Inoue et al., 2015). It is noteworthy that perturbing spike generation at the 
cell body does not achieve the same goal, because all efferent synapses are affected in that case.

Optogenetic experiments have been mainly restricted to small animals (rodents and insects). To maximize the potential of optogenetics for studying human cognition and behavior, studies of the cognitive function of the brain based on NHPs are rapidly progressing. The initial NHP optogenetic studies used optical stimulation to activate neurons in the primary motor cortex (M1) and frontal eye field (FEF) (Han et al., 2009; Diester et al., 2011). Even though these two primate studies had only reported modulation of local single-cell activity with no behavioral effects. Subsequent efforts provided support to the notion that optogenetics can be successfully used to manipulate behavior in NHPs (Cavanaugh et al., 2012; Jazayeri et al., 2012; Gerits and Vanduffel, 2013). Since then, studies using optogenetic approaches have provided new insights about the function and dysfunction of specific brain circuits in NHPs (Ruiz et al., 2013; Afraz et al., 2015; Inoue et al., 2015; Nassi et al., 2015; Acker et al., 2016; Galvan et al., 2016; Stauffer et al., 2016; El-Shamayleh et al., 2017; Tamura et al., 2017; Fetsch et al., 2018).

\section{6 - Outline of the thesis}

In this dissertation, state space methods were applied to study spiking activity of neurons in two brain areas of rhesus monkeys, namely PMd and PRR, both known to be involved in the planning of reach movements. We show that, by exploiting the neural state space, the computations could be readily identified, even without pre-selecting neurons based on tuning properties. We investigated and compared 
PMd and PRR neural dynamics that occur during movement planning and the subsequent dynamics that translate planning activity into movement activity.

To investigate the causal relationship between PMd and PRR during visuomotor transformations, an optogenetic approach was developed and used in this research. We examined how spatial tuning properties of PRR neurons are influenced by optogenetic-silencing the neural input from PMd. Specifically, we studied whether the optogenetics inhibition affects information processing during visuomotor transformation. 


\section{2 - Original Manuscripts}

This chapter contains the following manuscripts:

1. Kuang S, Guo H and Gail A. Shared preparatory tuning representation but distinct peri-movement neuronal dynamics in monkey frontal and parietal reach areas.

2. Guo H, Kuang $\mathrm{S}$ and Gail A. Reconfiguration of population dynamics for context-dependent sensorimotor transformations.

3. Guo H and Gail A. Optogenetic inhibition of premotor-to-parietal projections in rhesus monkeys reveals a causal role in rule-based sensorimotor transformations.

Author's contributions:

1. S.K. and A.G. designed the experiment. S.K. collected the data. S.K. and H.G. did the analyses. S.K., H.G. and A.G. wrote and edited the manuscript.

2. S.K. and S.W. designed the experiments and collected the data. H.G. did the analyses and wrote the manuscript.

3. H.G. and A.G. designed the experiment. H.G. collected the data, did the analyses and wrote the manuscript. Alexander Gail, Stefan Treue, Hansjörg Scherberger, Jens Gruber, Michal Fortuna, Janina Hüer and Hao Guo worked in a team to develop the optogenetic platform and discussed the experiment design. Michal Fortuna performed the histology experiments. 


\section{1 - Shared preparatory tuning representation but distinct peri- movement neuronal dynamics in monkey frontal and parietal reach areas}

PPR has previously been shown to co-encode both extrinsic visual goals and, predominantly, intrinsic physical movement goals in a reversing-prism task. To test functional differences between frontal and parietal areas, we compared spatial encoding in PMd and PRR in behaving monkeys performing reach movements while viewing through an optical reversing-prism. In the reversing-prism task, rhesus monkeys planned reaching movement with perturbed anticipated visual feedback once the movement initiated. Our results showed that both PMd and PRR were predominantly selective for physical, not visual goals during movement planning. Yet, frontoparietal areas differed during planning-to-execution transition. PMd exhibited larger peri-movement neural heterogeneity than PRR, resulting in a larger proportion of PMd neurons with either diminished or reversed spatial selectivity and more strongly changing neural state dynamics when transitioning from planning to execution. 


\title{
Shared preparatory tuning representation but distinct peri-movement neuronal dynamics in monkey frontal and parietal reach areas
}

\author{
Shenbing Kuang ${ }^{1}$, Hao Guo ${ }^{2,3}$, Alexander Gail ${ }^{2,3,4}$ \\ ${ }^{1}$ State Key Laboratory of Brain and Cognitive Science, Institute of Psychology, \\ Chinese Academy of Sciences, Beijing, China \\ ${ }^{2}$ German Primate Center, Göttingen, Germany \\ ${ }^{3}$ Georg-August-Universität Göttingen, Göttingen, Germany \\ ${ }^{4}$ Bernstein Center for Computational Neuroscience, Göttingen, Germany
}

\section{Abstracts}

Neural responses in dorsal premotor cortex (PMd) and parietal reach region (PRR) are modulated by spatial parameters of pending reach goals. PPR has previously been shown to co-encode both extrinsic visual goals and, predominantly, intrinsic physical movement goals in a reversing-prism task. In PMd, instead, predominant visual goal encoding was found using a joystick-cursor task. To test whether the seeming discrepancy indicates functional differences between frontal and parietal areas, we compared spatial encoding in PMd and PRR in the same monkeys under identical reversing-prism task conditions. Both PMd and PRR were predominantly selective for physical, not visual goals, with a ratio close to 3:1 in terms of fraction of neurons preferring one over the other during motor planning. Yet, during motor execution, PMd exhibited larger peri-movement neural heterogeneity than PRR, 
resulting in a larger proportion of PMd neurons with either diminished or reversed spatial selectivity and more strongly changing neural state dynamics when transitioning from planning to execution, particularly in visual goal neurons. We conclude that area PMd and PRR, from a representational perspective, share similar mixed neural selectivity for physical and visual goals during sustained reach planning under reversed vision, while from a dynamical perspective, PMd undergoes more dynamically changes of spatial encoding during transient motor execution.

\section{Introduction}

The primate dorsal premotor cortex (PMd) and parietal reach region (PRR) are both implicated in the planning of visually-guided arm reaching movements (Andersen and Buneo, 2002; Battaglia-Mayer et al., 2003; Crawford et al., 2011). Area PMd and PRR are interconnected (Johnson et al., 1996; Wise et al., 1997; Caminiti et al., 1999; Luppino et al., 2001; Marconi et al., 2001) and are shown to have similar motor-related, spatially selective modulation of single unit responses during reach planning (Gail et al., 2009; Westendorff et al., 2010; Klaes et al., 2011). Motor-related encoding in the frontoparietal reach network was identified with anti-reach paradigms in which the prospective motor goal is disentangled from the retrospective memories of visual instructions during movement planning (Crammond and Kalaska, 1994; Gail and Andersen, 2006). Motor goals can be specified in different ways, e.g. in different sensory domains, depending on the task demands (McGuire and Sabes, 2011). In visually guided reaching, motor goals can reflect either the required physical movement of the hand (physical goal), or the visual endpoint of the hand associated with this movement (visual goal). In 
typical visual-guided actions in our daily lives, both are intimately linked, unless mirror imaging or optical refraction (e.g. when reaching through a water surface) dissociate the visual feedback about our hand position from its actual physical movement. Here we compare frontal lobe area PMd and parietal area PRR of rhesus monkeys to ask whether visual or physical movement parameters predominantly determine neural motor-goal encoding during planning, and to characterize neural dynamics during reach execution under reversed vision.

To dissociate physical and visual goals in premotor area PMd, previous electrophysiology studies in rhesus monkeys used a joystick to control the movement of a computer cursor (or video image of the hand) with either congruent or reversed joystick-cursor mappings. In these studies, it was found that visual goal representations dominated over physical goal representations (ratio of physical vs. visual goal neurons 1:4) during action planning (Shen and Alexander, 1997; Ochiai et al., 2002, 2005) and during action execution (Schwartz et al., 2004). In contrast, a recent study from our lab used a reversing prism to disentangle physical and visual goal while still viewing one’s own actual hand (Kuang et al., 2016), and revealed a substantially larger fraction of physical goal neurons compared to visual goal neurons in parietal area PRR (ratio 3:1).

This marked difference in the visual versus physical goal encoding across studies could be attributable to (1) distinct visuomotor transformation processes in premotor and parietal cortices during action planning (Kalaska et al., 1997; Battaglia-Mayer et al., 2003; Pesaran et al., 2006; Desmurget and Sirigu, 2009; Gail et al., 2009; Chakrabarti et al., 2014), or (2) methodological differences between studies regarding real compared to representational (cursor or video) visual feedback (Bosco et al., 2010; Hawkins et al., 2013), or (3) potential confounds of visual memory. Here we compare spatial encoding characteristics of 
PMd and PRR neurons that were recorded under the same reversing-prism task and from the same monkeys (Kuang et al., 2016) to allow direct comparison of frontal and parietal areas. Further, by utilizing a combined reversing-prism anti-reach task (Kuang et al., 2016), we can disambiguate visual motor-related encoding from visual memory encoding to rule out confounds of visual memory or direct visual feedback (Shen and Alexander, 1997; Ochiai et al., 2002; Schwartz et al., 2004; Ochiai et al., 2005).

Previous studies comparing parietal and premotor movement preparation signals often focussed on extended planning periods during the instructed delay. The advantage of this is that motor command output and movement-related sensory feedback does not confound the planning signals of interest and that such quasistationary phase in behaviour allows easier quantification of neural selectivity over longer time windows. Yet, steady-state encoding in a closed-loop feedback system, like fronto-parietal sensorimotor loops, might make both “ends” of the loop look more similar than they actually are. Neural computation, instead, should predominantly happen during dynamic changes when the state of the system changes, e.g., due to processing of new input or the need to create a change in the output, and thereby could reveal differences between brain areas unnoticeable during steady-stage phases. In addition to single-neuron selectivity, we will also examine high-dimensional neural population dynamics (Shenoy et al., 2013) during the transition from movement planning to execution. In the framework of population dynamics, the cortical activity is better captured by a dynamical system than single unit tuning representations (Michaels et al., 2016). Along this line, neural activities in motor and premotor areas during planning have been interpreted as the initial state of a dynamical system whose evolution produces movement activity (Churchland et al., 2010; Churchland et al., 2012; Kaufman et al., 2014; 
Elsayed et al., 2016). Compared to (pre-)motor cortex, posterior parietal association areas lack spinal projections, but have more privileged access to multimodal sensory input from surrounding higher sensory areas (Bakker et al., 2012). Despite their interconnection, this could give rise to different dynamics of PRR and PMd during phases of non-stationary sensory input and voluntary action output. We will characterize and compare population dynamics in PRR and PMd under the same behavioural tasks and in the same animals to quantify how persistent or volatile the neural selectivity is between the planning and execution of movements, and to test in how far persistency/volatility depends on the frame of reference in which the information is encoded.

\section{Materials and Methods}

The animals, behavioral tasks, technical details of the apparatus, and experimental designs, including data acquisition and part of data analysis have been described in detail previously (Kuang et al., 2016). Below you will find a short summary of the methods common to the previous and current studies, and a detailed description of the new data and analyses. For the previous study, only data recorded from area PRR was available and analyzed on a neuron-by-neuron basis during the movement planning. Here we report unpublished data from area PMd, plus new aspects of the data from PRR, of the same animals and compare it quantitatively to pre-existing data from PRR with old and new methods.

All experimental procedures were conducted in accordance with European Directive 2010/63/EU, the corresponding German laws governing animal welfare, and institutional guidelines. 


\section{Apparatus and basic behavioral task}

We trained two male rhesus monkeys (Macaca mulatta; $\mathrm{F}$ and $\mathrm{S}$ ) to perform a visually instructed, memory-guided centre-out reach task on a fronto-parallel screen. The monkeys faced a liquid crystal display screen (19 inch ViewSonic LCD VX922; 5 ms off-on-off response time, refresh rate: $60 \mathrm{~Hz}$ ) covered with a transparent touch sensitive panel (IntelliTouch, ELO Systems, Menlo Park, CA) registering the position of the monkey's fingertips on the screen. In one dataset ("combined task", see below), monkey S was wearing active LED markers attached to its fingertips with a Velcro band and movements were additionally recorded by an optical motion tracking system (Visualeyez VZ 4000, PTI, Canada). The monkeys' visual field on the screen was approximately $10 \times 10 \mathrm{~cm}$ square, restricted by the monocular viewing of the screen through a tube-like aperture. The view of the screen for the other eye was blocked with a cardboard (for a schematic illustration, see Fig.1a of Kuang et al. 2016). The tube was either

embedded with a Dove prism (12.5 3 × $3 \mathrm{~cm})$ to achieve a high-quality left-right reversed view of the workspace (prism viewing context), or kept empty (normal viewing context). The distance between the display screen and monkeys' viewing eye was around $40 \mathrm{~cm}$. An infrared camera $(224 \mathrm{~Hz}$ CCD camera, ET-49B, Thomas Recording, Giessen, Germany) was used to monitor the pupil position of the non-viewing eye for gaze control.

The timeline for the reaching task was as follows (Fig. 1A): Each trial started after the monkey held hand fixation around the central white spot for a random time of $0.75-1.25 \mathrm{~s}$, a peripheral visual cue (5 $\mathrm{cm}$ eccentricity) was briefly flashed (cue period, $0.2 \mathrm{~s}$ ), indicating where the animal will have to reach later. Reach 
movements were not allowed to be executed until the "go" signal (the disappearance of central hand fixation spot) was given. During this delay period the monkeys had to keep the hand fixation and remember the location of the visual cue (1.0-2.0 s). After the "go" signal, the monkeys had to make a reach towards the previously cued location within a maximum of $1.5 \mathrm{~s}$. The visual cue reappeared at the same location to provide visual feedback after the monkey acquired the target, or after the maximum allowed movement period had expired. Eye fixation had to be kept throughout the course of the trial (tolerance window, $2 \mathrm{~cm}$ radius around fixation spot); otherwise, the trial was aborted immediately without reward. Liquid reward and acoustic feedback indicated correct (high pitch tone, reward) or incorrect (low pitch tone, no reward) behavior.

\section{Reversing-prims reach task designs}

We conducted two experiments. In the main experiment (reversing-prism task), two monkeys performed the center-out reach movements under either normal or reversed viewing contexts (Fig. 1B, top), allowing us to disentangle visual goal from physical goal. Monkeys could distinguish normal and prism contexts either by visually noticing the reversed feedback about their hand movements during acquisition of the fixation spot at the beginning of the trial, or when we switch between empty and prism-loaded aperture tube. Prism and normal trials were alternated in blocks of 40 trials (most recording sessions had four blocks, two in each context). The precise visual field alignment with and without prism was confirmed by consistent central gaze direction across both viewing contexts.

In the second experiment (combined prism-anti task), we combined the reversingprism task with an anti-reach task (Fig. 1B, bottom). In the main experiment, the 
locations of the current visual goal and the preceding visual instruction cue (aka visual memory) are identical. The second experiment served to further dissociate visual goal from visual memory encoding (Kuang et al., 2016). In the combined reversing-prism anti-reach task, there were two task rules (pro and anti) and two viewing contexts (normal and prism). The pro/anti task rules were instructed to the monkey by the colors of the central frame around the fixation spot (green: pro rule; blue: anti rule) during the cue period. The pro rule required the monkey to reach towards the visual cue position whereas the anti rule required reaching to the opposite of the visual cue location. Pro and anti trials were conducted either under the normal or the prism viewing context. Note that the reach task was defined in the monkey's visual coordinates in all task conditions. This means, in the prism pro trials, for instance, with a perceived right side visual cue, monkeys would need to physically reach to the left in order to bring the visual hand toward the visual cue location. In the prism anti trials, a perceived right-side visual cue would be associated with a physical rightward movement to bring the visual hand to the left (away from the perceived visual cue). Left and right cues and pro and anti trials were randomly interleaved from trial to trial. The combined reversing-prism antireach task was performed by monkey S only, as monkey $\mathrm{F}$ had to be excluded early from the experiment.

\section{Neural data acquisition}

In each experiment, we conducted extracellular recordings from area PMd and PRR (Fig. 1C) using up to five microelectrodes arranged in a concentric fashion in one five-channel microdrive ("mini-matrix"; Thomas Recording, Giessen, Germany) per each area. Pre-surgical structural MRI was used for the guidance of 
chamber placement (PMd, monkey F: $20 \mathrm{~mm}$ lateral, $20 \mathrm{~mm}$ anterior; S: $13 \mathrm{~mm}$ lateral, $17 \mathrm{~mm}$ anterior; PRR, monkey F: $7 \mathrm{~mm}$ lateral, $13 \mathrm{~mm}$ posterior; S: 6mm lateral, $10 \mathrm{~mm}$ posterior). Post-surgical MRIs verified the correct chamber positions and guided the recording penetrations. The raw signals were preamplified (20x; Thomas recording), band-pass filtered (154 Hz to $8.8 \mathrm{KHz}$ ), and amplified (400-800x; Plexon), before online spiking-sorting was conducted (Sort Client; Plexon). Additional to spike times, the spike waveforms were recorded (sampled at $40 \mathrm{KHz}$ ) and subjected to later offline sorting for the control of isolation quality (Offline Sorter; Plexon).

Since we recorded with multiple electrodes per recording session, we did neither preselect neurons nor adjust task parameters based on individual neural response profiles. All recorded and well isolated single units, regardless of task-related modulation properties, were recorded and included in the datasets. In total, we recorded six multi-day datasets, resulting in three datasets from each brain area. Each of the three datasets consisted of two datasets from the main experiment (monkey $\mathrm{F}$ and $\mathrm{S}$ ), and one additional dataset from the second experiment (monkey S only).

\section{Single neuron analyses}

To allow a direct comparison across brain areas, we employed similar analysis procedures as in our previous study (Kuang et al., 2016), but also applied additional methods.

To address the question of visual versus physical goal encoding during planning, we examined the sustained neuronal activity during the late delay period. For this we applied mainly single neuron selectivity (“tuning”) analyses to be compatible 
with earlier work and since this phase of the trial is characterized by almost stationary responses. Spatial selectivity was quantified by the average spike rate across trials in the last 800 ms before the "go" signal to capture the sustained planning activity. The direction selectivity index (DSI) was defined as contrast in spike rate (r) between left (L)- and right(R)-side cued trials:

$$
D S I=\frac{r_{L}-r_{R}}{r_{L}+r_{R}}
$$

The cue position was defined in the subject's visual field (i.e., viewed through the prism if present). The left-right direction selectivity was considered significant at $\mathrm{p}<0.05$ (t-test).

For datasets from the main reversing-prism experiment, we contrasted DSI across viewing contexts (normal vs. prism) to classify neurons into visual goal and physical goal. For the combined reversing-prism anti-reach task, we applied a similar two-step categorization procedure, of which the details can be found in the previous paper (Kuang et al. 2016). In brief, in the first step, we used the antidissociation (pro vs. anti) to identified motor goal neurons (as opposing to visual memory neurons); in the second step, we used the prism-dissociation (normal vs. prism) to categorize these motor goal neurons into visual goal and physical goal neurons.

To assess the neural dynamics in the transition from motor planning to motor execution we applied both, a single-unit categorization approach and a populationlevel neural state space approach. First, in the neuron-by-neuron categorization approach, for each neuron the directional selectivity (DSI) is quantified separately and then compared between the sustained delay period and the movement period. We defined the reach movement period as the $200 \mathrm{~ms}$ before reach target 
acquisition. This period captures the average movement time of about $120 \mathrm{~ms}$ in our tasks (approximation across data sets and task conditions) plus an estimated 80 ms delay from movement initiation to measurable lifting of the finger (Scott, 2004). For all neurons with significant DSI during the delay period, we categorized them into the following three subgroups based on their DSIs in the reach period: (1) Preserved neurons that maintained the same-sign directionality as in the delay period; (2) Not-tuned neurons that there are no longer significantly directional selective in the reach period; and (3) Reversed neurons that exhibited reversed-sign directionality with respect to the delay period. The categorization was done for each viewing context separately, to account for the fact that neurons depending on their spatial encoding properties during motor planning might have reversed directionality across viewing contexts.

Second, to avoid significance-based exclusion criteria for individual neurons and to achieve higher temporal resolution, we examined the neural dynamics of the population of recorded neurons in the high-dimensional neural state space (Shenoy et al., 2013; Elsayed et al., 2016).

\section{Neural population dynamics}

Spike-density functions (sample period: 10ms) were generated by convolving the raw spike trains of each trial with a Gaussian kernel (sigma: $50 \mathrm{~ms}$ ). The spike density functions were then sorted by experimental conditions and averaged across trials. The data was then grouped into the matrix $\mathbb{R}^{N \times C T}$, where $\mathrm{N}$ is number of recorded neurons, $\mathrm{C}$ is the number of conditions (normal viewing: left and right; prism viewing: left and right) and $\mathrm{T}$ is the number of sample time points (between $200 \mathrm{~ms}$ to $400 \mathrm{~ms}$ aligned to go-cue onset). The population response forms an $\mathrm{N}$ - 
dimensional space where each neuron represents one dimension and the state space trajectories are traced out over time. Principal component analysis (PCA) was applied to high-dimensional state space to reduce the dimensions to $\mathbb{R}^{k \times C T}$, where $\mathrm{k}<\mathrm{N}$. For our datasets, we projected the full dimensional data onto the first 10 principal component space $(\mathrm{k}=10)$, as they accounted for $>90 \%$ of the variance of the data in each dataset. Trajectories in neural state space are quantified in the space of these first 10 latent dimensions extracted through PCA.

To estimate the changes of direction selectivity from motor planning to execution, we defined a vector of selectivity (VOS). The VOS connects the state space trajectories of right- and left-side cued conditions between corresponding time samples. The VOS is computed separately in each viewing context (normal vs. reversed). We calculated the cosine value of the angle by which the VOS rotates over time. Temporal changes in the direction selectivity of single-neurons contribute to the VOS rotation angle, with the effect being accumulated as more units change their selectivity. Instead, a parallel movement of the VOS in the state space with time indicates that none of the neurons change their selectivity. Correspondingly, small angle changes in neural space denotes that most of neurons preserve their direction selectivity, while large angle changes indicate that most neurons change their direction selectivity substantially. The cosine values of the angle were calculated between the VOS at 200ms preceding the "go" cue (as a fixed reference) and the VOS at each subsequent time point in a time-resolved fashion.

Bootstrap tests ( $\mathrm{N}=1,000$ resamples) were used to estimate the variability of the cosine values. We resampled as many trials with replacement from each condition as were originally recorded in that condition. The cosine values were then calculated in the same manner presented above to get a pseudo-sample. To 
determine at each point if the temporal evolution of the VOS rotation in PMd and PRR are significantly different, we used the distribution of the difference in PMd and PRR pseudo-samples to construct a confidence interval for the difference in PMd and PRR cosine values. If the confidence interval doesn't include zero, we conclude that there is a significant difference in cosine values between PMd and PRR.

\section{Results}

\section{Datasets}

We recorded 256 PMd neurons and 362 PRR neurons from two monkeys (F: 116 PMd, 199 PRR, S: 140 PMd, 163 PRR) for the main experiment (reversing-prism task), and an additional dataset of 186 PMd and 81 PRR neurons from monkey S for a second experiment (combined prism-anti task). These datasets were collected after monkeys had been well trained and their performances reached high plateaus in each task condition.

Similar preferential encoding of physical goal vs. visual goal in area PMd and PRR during planning

We first examined directional selectivity of neurons (DSI) for either the visual goal or the physical goal in area PMd based on data from the reversing-prism experiment. Equivalently to our previous PRR findings, individual neurons during reach planning were directionally selective for physical goal information, others for visual goal information. Figure 2A shows the response profiles for two PMd example neurons, both of which were characterized by sustained activity during 
the delay period which was directionally selective in both the normal and reversed viewing contexts. According to the categorization approach (see Methods), the example in the left panel qualifies as physical goal (PG) neuron. Its delay activity was consistently higher when the physical goal location was on the right side, irrespective of visual goal locations. In contrast, the example in the right panel is a visual goal (VG) neuron, with an opposite pattern of spatial selectivity: the delay activity was higher either when the physical hand went rightward in normal trials or when the physical hand went leftward in prism trials. This means, the two more active conditions for the VG neuron had a rightward visual goal location in common, irrespective of the physical goal location.

The two example neurons differed not only in the spatial frame of reference of their directional selectivity, but also in other neural response properties. Specifically, the activity of the VG neuron during reach execution was clearly dissimilar from the delay period activity as it became more complex and idiosyncratic: the directional selectivity in the normal viewing context reversed between the delay and movement periods, and disappeared in the movement period of the prism context. Such temporal heterogeneity from delay to reach was observed frequently in our datasets. We will quantify below if these effects were specific to area PMd compared to PRR, specific to the prism compared to the normal viewing context, or specific to the VG compared to PG neurons.

PG/VG neurons are defined based on a coarse categorical classification, irrespective of common additional modulatory effects on response gain and selectivity. As in our previous PRR dataset, the range of selectivity profiles across neurons in PMd was broad, indicating a mixture of VG and PG frames of reference. The spread in the distribution of the direction selectivity indices (DSI) contrasted between normal and prism context confirms this view (Fig. 2B). Since DSI was 
calculated relative to the visual goal location, we expect VG neurons to have the same signed DSIs across contexts (populating the $1^{\text {st }}$ and $3^{\text {rd }}$ quadrant in Fig. 2B) while physical goal neurons have the opposite signed DSIs across contexts $\left(2^{\text {nd }}\right.$ and $4^{\text {th }}$ quadrant). The population distribution formed a continuous and diverse spectrum from visual goal encoding to physical goal encoding for PMd neurons from both monkeys. As previously observed in PRR, the distribution of DSI values could be explained neither by the assumption that all PMd neurons are selective for the physical goal (95\% confidence limit for this assumption shown as ellipse in Fig. 2B), nor by the opposite assumption of pure visual goal encoding. Nevertheless, the fraction of neurons falling into either of the two extreme categories can provide a hint on predominant encoding in each area.

PG selectivity overall was more common than VG selectivity in both brain areas. Table 1 summarizes the DSI analysis of PMd and PRR datasets showing the number and the fraction of neurons which were directionally selective in each viewing context. Of those neurons which were selective in both viewing contexts during the late delay phase (20-40\% across datasets), and hence were eligible for the VG/PG categorization, about 2/3 (61-79\%) were PG neurons, about 1/3 (2139\%) were VG neurons in both PRR and PMd (monkey F, area PMd, PG selectivity: 61\% (28/46); S-PMd-PG: 75\% (21/28); F-PMd-VG: 39\% (18/46), SPMd-VG: 25\% (7/28)). A chi-square independence test on the number of VG and PG neurons showed that the proportion of these two neuron types did not differ between PMd and PRR in either monkey ( $F: p=0.18$; $\mathrm{S}$ : $\mathrm{p}=0.17$ ). This means, in both brain areas of either animal, we observed an intermingled encoding of visual and physical reach goal information, with the latter being more predominant.

In addition, the prevalence of physical versus visual goal representation was also confirmed by using dPCA approach (Kobak, 2016). The subspaces that embedded 
either PG or VG representation during the sustained delay period were separated. The first dimension of PG subspace captured more variance compared to the first dimension of VG subspace in PMd (monkey F, area PMd, PG variance: 36.1\%; SPMd-PG: 31.6\%; F-PMd-VG: 20.9\%; S-PMd-VG: 27.1\%).

\section{Visual goal encoding is not confounded by visual memory encoding}

As noted above, the location of the visual goal matched the location of the visual cue in the reversing-prism task, which was also the case in previous PMd studies of other labs using joystick-cursor tasks (Shen and Alexander, 1997; Ochiai et al., 2002; Schwartz et al., 2004; Ochiai et al., 2005). This means, the visual goal neurons could in principle encode visuospatial memory of the instructive cue. To rule out this possibility, we combined the reversing-prism task with an anti-reach task (Kuang et al., 2016). We thereby can spatially dissociate visual memory, visual goal and physical goal (Fig. 1B). This test could only be performed in monkey S since monkey $\mathrm{F}$ had to be excluded from the experiment for reasons unrelated to the study.

Neurons in PMd are not encoding visual memory of the cue. Opposite-sign DSIs between pro- and anti-reach trials in both the normal (Fig. 3A) and the prism (Fig. 3B) viewing contexts confirmed that directional selectivity of individual neurons correlates with the motor goal $\left(2^{\text {nd }} \& 4^{\text {th }}\right.$ quadrants $)$ rather than visual memory $\left(1^{\text {st }}\right.$ $\& 3^{\text {rd }}$ quadrants). DSI values in the anti-dissociation distributed narrowly along the “motor goal” diagonal ( $2^{\text {nd }} \& 4^{\text {th }}$ quadrants) in both viewing contexts (Fig. 3A-B), indicating pure motor-goal encoding independent of visual memory. Of all 45 classifiable neurons (significant DSIs in both pro and anti conditions), 96\% (43/45) were motor-goal related, while not more neurons than expected by chance $(4 \%$, 
2/45) were visual-memory related. This confirms that, like PRR neurons, PMd neurons during motor planning are generally motor-goal related.

In a second step, we contrasted DSIs between normal and prism trials (prismdissociation) in both the pro- (Fig. 3C) and the anti-reach (Fig. 3D) trials, equivalently to the two datasets presented above. Different to the anti-dissociation, the distribution of DSIs in the prism dissociation showed an intermingled diverse distribution of both physical-goal and visual-goal related neurons (Fig. 3C-D). Of the 43 motor goal neurons identified above, 19\% (8/43) were further classified as visual goal neurons and 30\% (13/43) were classified as physical goal neurons. The remaining 51\% (22/43) were undefined motor goal neurons since their DSIs did not reach significance concurrently in both the normal and prism conditions. Note that a considerable fraction of neurons dropped out in this approach because of the stringent criterion we imposed on the neuron classification procedures (see Materials and Methods).

In summary, with the help of the combined reversing-prism anti-reach task we found that neural selectivity during the delay period in PMd is motor-related, not confounded by visual memory encoding, with a prevalence of physical over visual goal encoding. These results are consistent across all three datasets and equivalent to the previous observations in PRR (Fig. 4).

\section{PMd exhibited larger neural heterogeneity than PRR during planning-to- execution transition}

The two example neurons from PMd in Fig. 2A differed with respect to the consistency of neural tuning over time. The left example neuron shows stable encoding of the physical goal during planning and execution, with increased 
modulation strength during execution. The right example neuron switches from visual goal encoding (gain-modulated by viewing context) to an undefined frame of reference. To explore the evolution of neural activity from planning to execution more quantitatively, we characterized the neural heterogeneity over time in PMd neurons and compared them with PRR neurons in two separate ways (see Materials and Methods).

First, taking the neuron-categorizing approach, we counted the frequency of neuron subtypes for each viewing context, each monkey and each brain area (Table 2). We restricted this analysis to neurons that had significant delay period DSIs. We found that a portion of neurons from both brain areas either lost or altered their directionality when transitioning from delay to reach epochs. In PMd, on average 39\% (range: 28\%-51\%) of the examined neurons had preserved directionality, 19\% (15\%-24\%) had reversed directionality and 42\% (34\%-54) lost their directionality. In PRR, on average 57\% (52\%-62\%) of the examined neurons maintained their directionality, while 14\% (9\%-20\%) reversed their directionality and 29\% (19\%$39 \%$ ) became spatially non-selective. Chi-square tests of independence on the frequencies of neural subtypes indicate that the patterns of neural transition differed between PMd and PRR in both viewing contexts in monkey S (normal: $\mathrm{p}=1.6 \times 10^{-7}$; prism: $\mathrm{p}=1.5 \times 10^{-4}$ ) and under the normal viewing context in monkey $\mathrm{F}$ $(\mathrm{p}=0.01)$. In addition, the viewing contexts had significant influence on the frequency of individual neural subtypes in area PRR (F: $\mathrm{p}=0.0029 ; \mathrm{S}: \mathrm{p}=0.018$ ) but not in area PMd (F: p=0.59; $\mathrm{s}$ : p=0.1), with higher fractions of PRR neurons becoming not tuned during the movement period in the prism context (F: 39\%; S: 36\%) than the normal context (F: 19\%; S: 22\%).

The dynamic change in neural selectivity in the transition from planning to execution was more prominent in VG than in PG neurons in each dataset. As 
summarized in Fig. 5, for VG neurons, on average 38\% (PMd-F: 39\%; PMd-S: 27\%; PRR-F: 36\%; PRR-S: 49\%) of the examined neurons had preserved directionality, while 28\% (PMd-F: 22\%; PMd-S: 34\%; PRR-F: 36\%; PRR-S: 19\%) had reversed directionality and 34\% (PMd-F: 39\%; PMd-S: 39\%; PRR-F: 28\%; PRR-S: 32\%) lost their directionality. In contrast, for PG neurons, on average 61\% (PMd-F: 66\%; PMd-S: 36\%; PRR-F: 72\%; PRR-S: 68\%) had preserved directionality, while 12\% (PMd-F: 14\%, PMd-S: 19\%; PRR-F: 5\%; PRR-S: 11\%) had reversed directionality and 27\% (PMd-F: 20\%, PMd-S: 45\%; PRR-F: 23\%; PRR-S: 21\%) lost their directionality. A Chi-square test of independence revealed that the frequency distribution of directionality change differed significantly between VG and PG neurons $(\mathrm{p}<0.001)$. This means, during the transition from reach planning to reach execution, VG neurons are less likely to preserve their directional selectivity than PG neurons. In other words, VG neurons show larger neuronal dynamics than PG neurons during the transition.

In a second approach, we examined the temporal evolution of planning-toexecution transition using PCA-based high dimensional state space analysis. All quantitative analyses were conducted in the 10-dimensional space (see Materials and Methods). Yet, for a more intuitive understanding of the differences between PMd and PRR, we visualized the temporal evolution of the neural states in the three-dimensional space which was spanned by the top three dimensions and which explained more than $70 \%$ of the total variance in the data. After the go-cue onset, in the transition from movement planning to execution, neural trajectories evolved through state space in both brain areas, but with different characteristics (Fig. 6A). The vector that connects the state space trajectories of right- and left-side cued trials at each time point reflects the instantaneous direction selectivity of the neural population (and was therefore termed vector of selectivity, VOS). Temporal 
change in direction selectivity of single-neuron is reflected in the time-resolved VOS rotation angle. The effect of single-neuron on VOS rotation is accumulated as more units change their selectivity. Since VOS moves along condition-invariant dimensions, quantitative VOS rotation is independent of the temporal dynamics shared across all conditions. Differences between brain areas become visible by the fact that the VOS in PMd over time stays less parallel to the VOS before go-cue onset (Fig. 6A). VOS rotation was quantified using the cosine value of angle (see Materials and Methods). A smaller cosine value corresponds to a larger VOS rotation, indicating change in selectivity during planning-to-execution transition. We found that the cosine values immediately after go-cue onset were high for both areas, because selectivity was still correlated well with selectivity during the delay period. Starting from around 100ms after go-cue onset, VOSs rotated steadily for both areas as the trial proceeded. This VOS rotation revealed significantly larger dynamical changes in PMd than in PRR (Bootstrap test, see Materials and Methods) starting from around 100-150ms in monkey S, and 180-250ms in monkey F (Fig. 6B, 6C). The PMd results are consistent with recent research that proved the orthogonality between the preparatory and movement subspaces (Elsayed et al., 2016).

Taken together, our results indicate that in both frontal and parietal areas, similar preparatory activity during the delay period can transition to idiosyncratic patters of peri-movement activity. The spatial selectivity during movement execution period could be maintained, reversed, or lost in individual neurons. This temporal heterogeneity was higher in frontal PMd than parietal PRR, in both the normal and the prism viewing context. This means that the planning-to-execution nonstationarity of the spatial reference frame is more prominent in area PMd than area 
PRR, is more prevalent in VG neurons than PG neurons, but not specific to movements under reversed visual feedback.

\section{Discussion}

In the current study we obtained three main findings. Firstly, area PMd had a preferential encoding of physical goal versus visual goal during reach planning under reversed vision. This spatial encoding property was quantitatively very similar between PMd and PRR neurons. Secondly, by combining reversing-prism with anti-reach task in a control experiment, we confirmed that encoding of visual goal in PMd was not confounded by visual memory, similar to PRR neurons (Kuang et al., 2016). Thirdly, although PMd and PRR showed remarkably similar spatial selectivity during sustained motor planning, PMd exhibited larger neural heterogeneity and dynamics during the transition from motor planning to motor execution, an effect for which mostly visual goal neurons were responsible. We conclude that PMd and PRR have similar, mixed spatial goal selectivity during the planning phase but different neuronal dynamics during planning-to-execution transition. These shared preparatory representation and distinct peri-movement population dynamics suggest that parietal and frontal reach areas likely serve differential functional roles during sensorimotor transformation: while parietal PRR is more suitable for stable goal representations, frontal PMd is more engaged during goal-to-movement implementation.

Frontal and parietal reach areas share similar predominant encoding of physical goal relative to visual goal under reversed vision 
We found that majority of PMd neurons encodes physical goal information while only a minor fraction encodes visual goal information. The proportion of physical goal and visual goal neurons was quantitatively very similar between frontal PMd and parietal PRR (Table 1). Previous studies have shown that PMd and PRR have shared neuronal properties on the sustained motor goal representations during reach planning (Kalaska, 1996; Gail et al., 2009; Westendorff et al., 2010; Klaes et al., 2011). Our current data not only was consistent with this idea and also extended this similarity to the next level. We showed that, as the two subdivisions of motor goal encoding, visual goal and physical goal representations were also very similar between area PMd and PRR. Our data supports the notion of a common neural representation in the frontal and parietal circuits during action planning. Similar spatial representations indicate that reach planning was an emerging property of the tight mutual interaction and coordination in the frontoparietal network (Pesaran et al., 2008).

On the surface, the observation of prevalent physical goal encoding in PMd neurons is incompatible with the findings from several previous studies reporting predominant visual goal representations in dorsal and ventral premotor cortex (Shen and Alexander, 1997; Ochiai et al., 2002; Schwartz et al., 2004; Ochiai et al., 2005). However, we should be aware that the current study and previous studies employed visuomotor tasks that differed in several important ways. In the previous studies, monkeys controlled the movements of a computer cursor (or static hand image) by manipulating a joystick. By implementing either directionally congruent or incongruent joystick-cursor relations the authors could separate visual target from planned physical movement. In contrast, the current study dissociated visual and physical movements using reversing-prism. The monkeys in our task performed arm reaching movements while viewing one's own actual hand 
(reversed in some trials, though). Firstly, the types of movements might be different between studies. The joystick-cursor tasks often involve wrist rotations or small-amplitude hand displacements that are clearly not identical to the veridical arm reaching movements conducted in the reversing-prism task. Secondly, the visual target (cursor) and the physical movement (joystick) were operating on the dissociated work planes in the cursor task, while in the current prism task they were within the same workspace because the visual hand representation is a direct visual consequence resulted from physical movement. Thirdly, and perhaps most critically, the visual feedback to the monkeys about their hand movement was distinct between studies. The visual feedback in the cursor task was artificial cursor (or static hand image) shown on a projected screen. In contrast, in the current study monkeys could view a veridical (though reversed) representation of their paws associated with the physical movement.

These differences in the movement types (wrist/hand vs. arm reaching), visual feedbacks (artificial vs. veridical) and workspaces (dissociated vs. aligned) might render monkeys with different levels of sense of action agency and therefore elicit different neural representations. Specifically, in the reversing-prism task, monkeys might have stronger sense of motor agency and therefore we observed a preferential encoding of physical goal during planning. In contrast, in the cursor task where monkeys couldn't view their hand directly, they might rely mainly on the visual representation to perform the task. Correspondingly, PMd showed a predominant encoding of visual goals. To validate this speculation would require further experiments to implement both reversing-prism and joystick-cursor tasks in the same animals. Nevertheless, our current results suggest that precautions should be exercised when comparing sensorimotor functions under distinct behavioural tasks. Here, by contrasting parietal and frontal responses under the matched task 
and in the same animals, we showed that frontal and parietal reach areas exhibited similar predominant of physical goal representations during movement planning under reversed vision.

\section{Frontal and parietal reach areas have distinct planning-to-execution neural dynamics}

Delay period activity in the frontoparietal reach network has often been considered to be a precursor (preparation) for the subsequent movement, e.g. encoding the motor error in a particular spatial frame of reference (Kalaska, 1996; Wise et al., 1997; Andersen and Cui, 2009; Crawford et al., 2011). While spatial encoding in parietal cortex might be subject to changes according to the cognitive demands of the task prior to motor-goal specification (Archambault et al., 2011; Bremner and Andersen, 2014), this view implies that neural responses maintain their selectivity when the subjects transition from the motor planning period to the movement execution period (Buneo et al., 2008; Lehmann and Scherberger, 2013). The fact that the (successful) movement itself progressively reduces the motor error to zero might diminish spatial selectivity during execution (Bremner and Andersen, 2014), but does not mandate a change in reference frame. On the other hand, visual and somatic sensory feedback as well as motor output dynamically change during movement execution and can give rise to substantial neural dynamics, as observed in PMd and M1 (Churchland and Shenoy, 2007; Churchland et al., 2010; Churchland et al., 2012; Kaufman et al., 2014; Elsayed et al., 2016). Different to previous studies on spatial reference frames, our manipulation of the visual feedback becomes mainly effective during motor execution, not during motor-goal instruction, yet influences the planning activity. 
Here we showed that despite complex and heterogeneous temporal dynamics of single-neuron activity, neural activity in PRR is endowed with a population-level coding of physical goal that is more stable and robust from planning to movement, whereas, PMd exhibits much larger changes in terms of selectivity. The result is consistent with the conceptual model prediction that the parietal area plays a dominant role in stable goal representations (state estimator, for both planning and executing epochs), while the premotor area plays a dominant role in the implementation of planning-to-execution transitions. This cross-area difference implies that the highly similar and concurrent activation in the frontoparietal network during movement planning should not be viewed as a sign of redundancy but could be more appropriately interpreted as a vital element of distributed processing during visuomotor transformation.

Our results of more pronounced neural heterogeneity during planning-to-execution transition in PMd than PRR indicate that area PMd is more tied to the physical aspect of the movement while area PRR is more involved in the abstract goal representations. This view is consistent with several lines of recent studies. For instance, reference frame studies have showed that motor goal representations in PRR are predominately eye-centered (Batista et al., 1999; Buneo et al., 2002; Chang and Snyder, 2010; McGuire and Sabes, 2011) whereas in PMd more handcentered (Pesaran et al., 2006). Electrical micro-stimulations in human PPC induced strong urges to move various body parts without overt movement execution. In contrast, stimulations in premotor areas introduced overt unconscious movement execution (Desmurget et al., 2009). A more recent study also reported markedly different patterns of neural synchronization between PMd and PRR, indicating different functional organizations (Chakrabarti et al., 2014). Here, we added to these cross-area differences by reporting different neural transitions from 
reach planning to reach execution. Our data of similar goal representations but distinct peri-movement dynamics between frontal and parietal reach areas support the notion of shared and specialized visuomotor processes implemented in multiple visuomotor areas for the planning and execution of visually guided movements.

\section{Alternative interpretation of visual goal and physical goal representations in the frontoparietal network}

The sustained spatial selectivity during the delay period in the frontoparietal network is often interpreted as 'motor goal' encoding (Kalaska, 1996; Snyder et al., 1997; Gail and Andersen, 2006). In the current study, we labeled the two ends of the otherwise continuous range of observed spatial encoding schemes as 'visual goal' and 'physical goal', to conform to the previous nomenclature. Yet, the immediate task parameter we manipulated with the optical prism was the upcoming visual feedback about the planned movement. In this sense, the visual goal encoding might represent the anticipated visual feedback once the movement unfolds. We argue that one might still interpret the observed spatial selectivity as "motor goal", but one would have to assume then that motor goals inherently contain information about the desired or the anticipated visual sensory consequences of a movement (Desmurget and Sirigu, 2009; Waszak et al., 2012). In the current study, the reach task was defined by a combination of the desired visual feedback about the movement ('Where do I want my hand to visually be at the end of the trial?') and the required physical movement itself ('Which movement is needed to achieve this?'). A motor-goal encoding areas like PMd and PRR should therefore contain representations of both aspects of the planned movement, since both are relevant for conducting the required movements properly. 
In this alternative interpretation, the visual goal neurons could be viewed as encoding for the anticipated visual consequence of intended movement. One possible explanation for more physical goal neurons than visual goal neurons in our study could be that, some (if not all) of physical goal neurons are the proprioceptive anticipation of upcoming reach movements, and they overlapped with the true planed physical movement. The direction of "physical goal" in our experiment could not be dissociated from the anticipated proprioceptive feedback about the upcoming reach. We could not tell them apart with the current design, but it is highly unlikely that sensory anticipations operate exclusively in the visual domain. Hence, the most parsimonious, yet at this point speculative interpretation of our data would be that all motor related neurons encoded anticipated sensory feedback, some in the visual domain, the other in the proprioceptive domain, with variable degree of overlap.

Why would sensorimotor cortex encode the anticipated visual and/or proprioceptive aspects of upcoming movement during the planning period? A major functional relevance of anticipatory encoding of sensory action effects during motor planning lies in its potential to contribute to action selection (Hommel et al., 2001; Waszak et al., 2012). An intended motor plan then is selected based on the desired sensory effects of an action. Anticipating visual sensory aspect of a movement plays a role in various concepts of motor cognition, like the ideomotor concept (Shin et al., 2010), motor imagery and mental rehearsal (Jeannerod, 2001), motor awareness (Desmurget and Sirigu, 2009), and perceptual stability (Duhamel et al., 1992). Also, the idea of representing future sensory parameters of a movement is reminiscent of internal models in optimal motor control, a function that PPC has previously been associated with during action execution (Mulliken et al., 2008; Shadmehr et al., 2010; Franklin and Wolpert, 
2011). The intriguing findings here would be that area PMd and PRR co-represents

the intended physical movement and its associated visual sensory effect already during reach planning, which marks a conceptual difference to the sensory forward predictions during motor control.

The sensory anticipation interpretation adds a new complementary perspective to the current understanding of spatial representations in the frontoparietal circuits. They shed a new light on the concept of motor goal, suggesting that the formation of a motor goal implies not just the preparation of a proper physical motor command and its representation in different spatial reference frames, but also the anticipation of visual sensory aspects of the future movement.

\section{Figure captions:}

Figure 1. Task design and experimental procedures. (A) The timeline of a typical delayed center-out reach movement. Monkeys maintained ocular fixation (central red spot) throughout the trial and conducted hand reach movement towards the memorized visual cue location after the "go" instruction (disappearance of central white spot). (B) 2x2 task conditions. The reach movement should be performed under either the normal or the reversed viewing contexts (main experiment), therefore dissociating intended visual hand movement (visual goal) from physical hand movement (physical goal). In a second experiment, movements under the normal and prism viewing contexts could be required to reach either towards (pro rule) or to the opposite location of (anti rule) the visual cue location. The Pro vs. anti comparison dissociated visual cue location from the visual and physical hand movements. Together, the combined reversing-prism anti-reach paradigm unambiguously disentangled the spatial encodings of visual memory, visual goal 
and physical goals during the delay period. (C) We conducted extracellular recordings of single-unit spiking activity from dorsal premotor area (PMd) and parietal reach region (PRR) while monkeys performed the tasks (regions of interest shown for monkey S).

Figure 2. Co-existence of visual goal and physical goal representations in area PMd neurons. (A) The raster plots (trial-by-trial spike events) and the mean neural responses of example physical goal neuron (left panel) and visual goal neuron (right panel) in each viewing context (normal: green; prism: red) and in each direction (visual cue left: dashed; right: solid). The directional selectivity of delayperiod activity correlated with the direction of either physical movement (physical goal neuron) or visual movement (visual goal). (B) Classification of physical goal and visual goal neurons at the population level in each monkey. Dashed ellipses denote the confidence limit within which $99 \%$ of the surrogate data falls when assuming purely physical goal encoding as the null hypothesis.

Figure 3. Visual goal and physical goal encodings in PMd neurons were not confounded by visual memory encoding, as confirmed by the combined reversingprism anti-reach experiment. (A-B) DSI values between pro and anti reaches were strongly anti-correlated, indicating almost exclusive motor-related encoding during the delay period in both the normal (A) and the prism viewing contexts (B). (C-D) Of all motor-related neurons (non-* symbols) identified in the above antidissociation, 19\% were classified as visual goal neurons (triangles) and 33\% as physical goal neurons (squares) when contrasting DSI between normal and prism trials. Many neurons were unclassifiable in first anti-dissociation or in the second prism-dissociation because DSIs did not fulfill the stringent classification criterion (see Methods). Note, (C) and (D) contain the same data as (A) and (B), but contrasted differently. 
Figure 4. The ratio of physical goal vs. visual goal neurons in each brain area and in each dataset. For all three datasets, area PMd and PRR showed similar prevalence of physical goal vs. visual goal encodings.

Figure 5. The frequency distribution of planning-to-execution neural transitions for VG and PG neurons, respectively. Data were collapsed across datasets and brain areas. Note that VG neurons had a lower probability of preserving their direction selectivity than PG neurons during the transition.

Figure 6. Large neural population dynamics in PMd than PRR revealed in neural state space. (A) Low-dimensional representation of neural population activity in [200ms 400ms] interval aligned to go-cue onset for two example datasets (S-PMd, S-PRR). Population trajectories are plotted in coordinates defined by the first three principal components of each dataset. Solid and dashed lines represent the neural trajectories of right- and left-cued condition respectively. The vector connecting left- to right-side cued trajectory (VOSs) along total 600ms are represented as two color-coded manifolds, based on that VOS is before (dark) or after (light) go-cue. Each neural trajectory is marked by dots with $100 \mathrm{~ms}$ intervals. The VOSs at $0 \mathrm{~ms}$, 200ms and 400ms are emphasized as black arrows, which are corresponding to the asterisk marks in (B). (B) Dynamical changes of VOSs in PMd and PRR are estimated as cosine values of angle when aligned to go-cue onset (see methods). Line represents the change of VOSs across time. Shaded area indicates the confidence interval $\left(2.5^{\text {th }}\right.$ and $97.5^{\text {th }}$ percentiles of the values generated by the bootstrapping procedure), and horizontal line along the bottom of plot denotes times when area PMd exhibits more substantial dynamical change comparing to PRR. (C) Same as (B), but for combined prism-anti task, monkey S. 


\section{Table captions:}

Table 1. Neuron categorization based on the delay period activity in each brain area for each monkey in the main reversing-prism experiment. Area PMd and PRR have similar percentages of PG and VG neurons.

Table 2. Frequency analyses of directionality from planning to execution in each viewing context each monkey and each brain area. Note that the two datasets from monkey $\mathrm{S}$ are pooled. While more PRR neurons preserved their directionality during the transition, more PMd neuron either reversed or lost their directionality. 
Figure 1

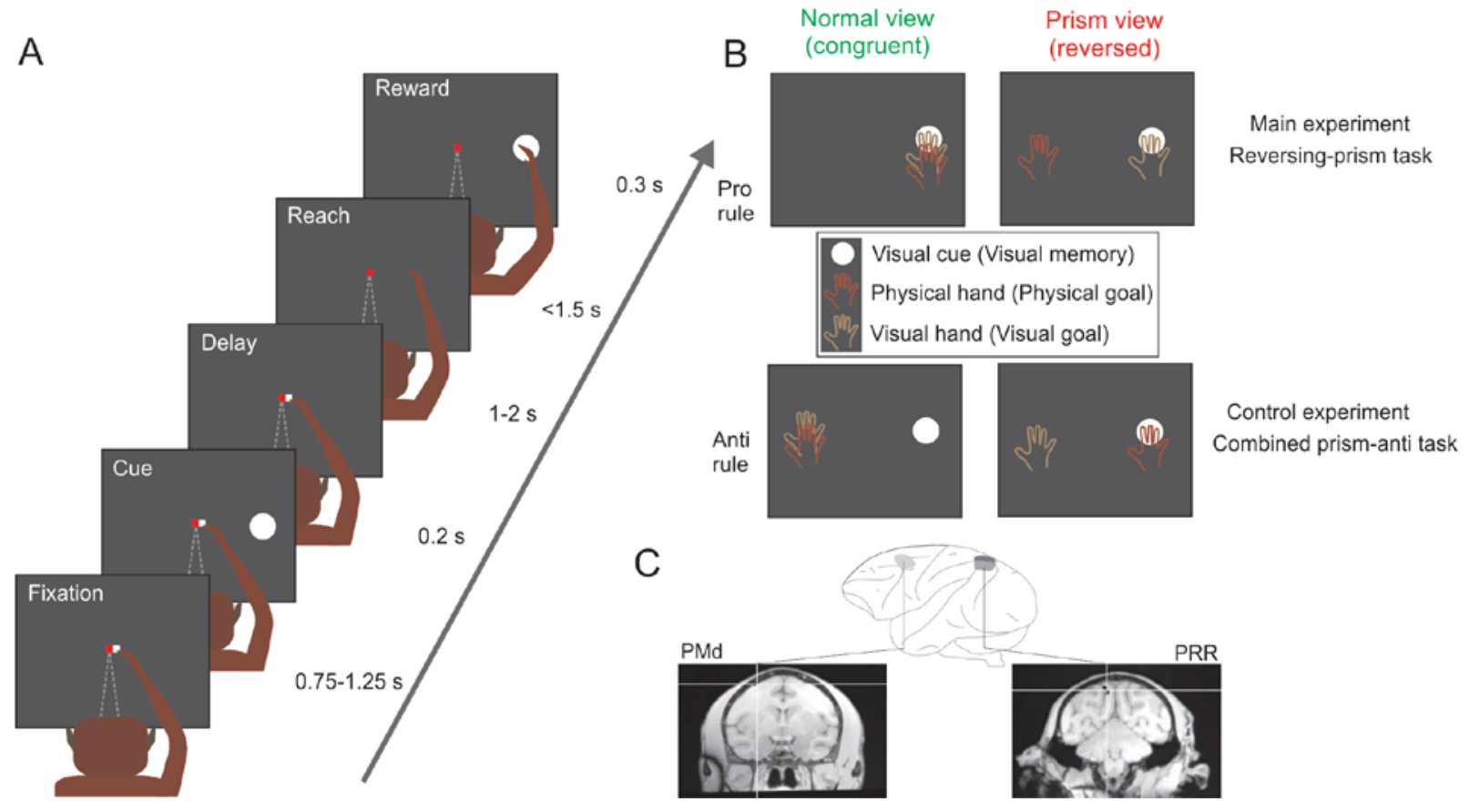


Figure 2
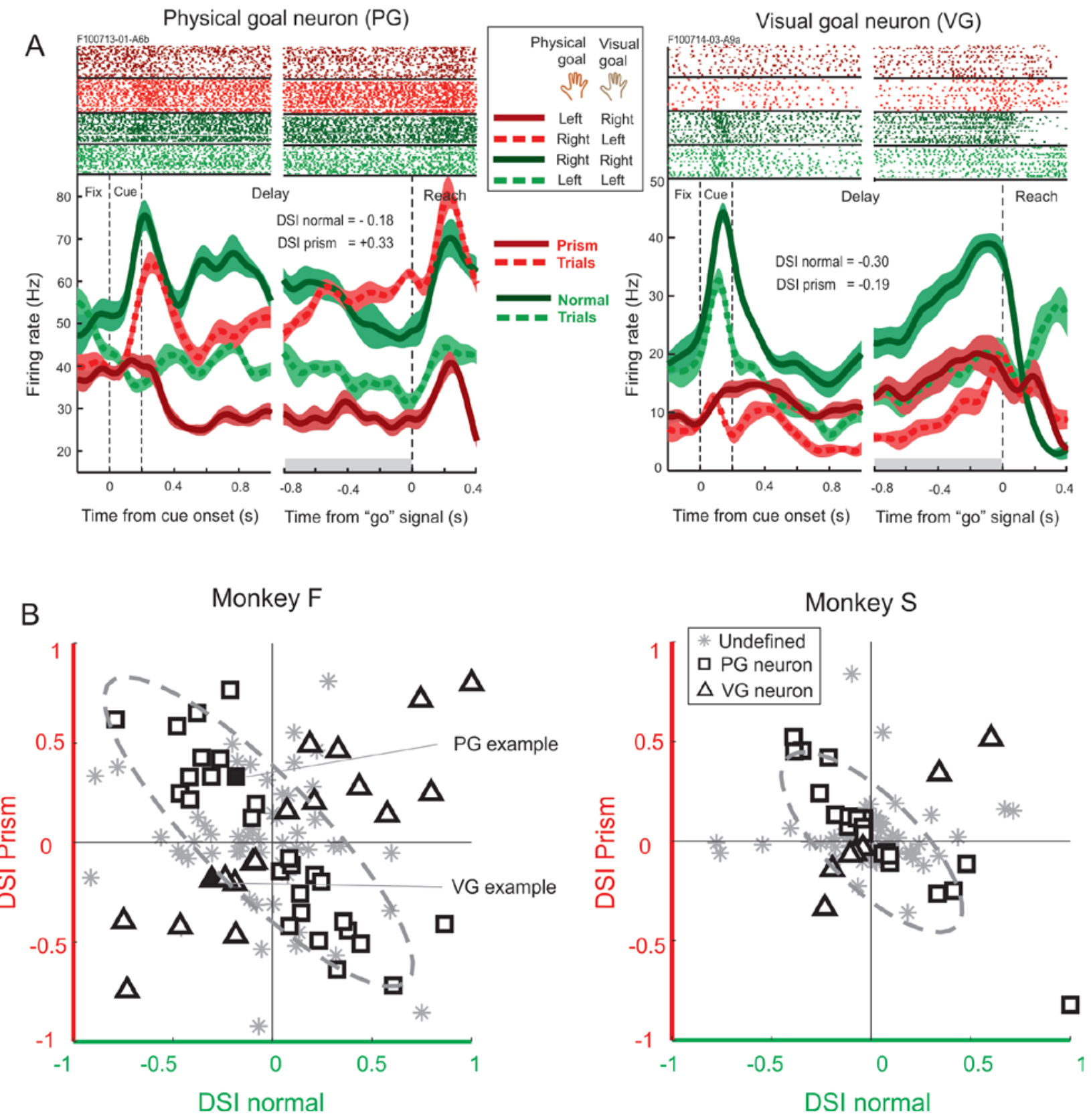
Figure 3
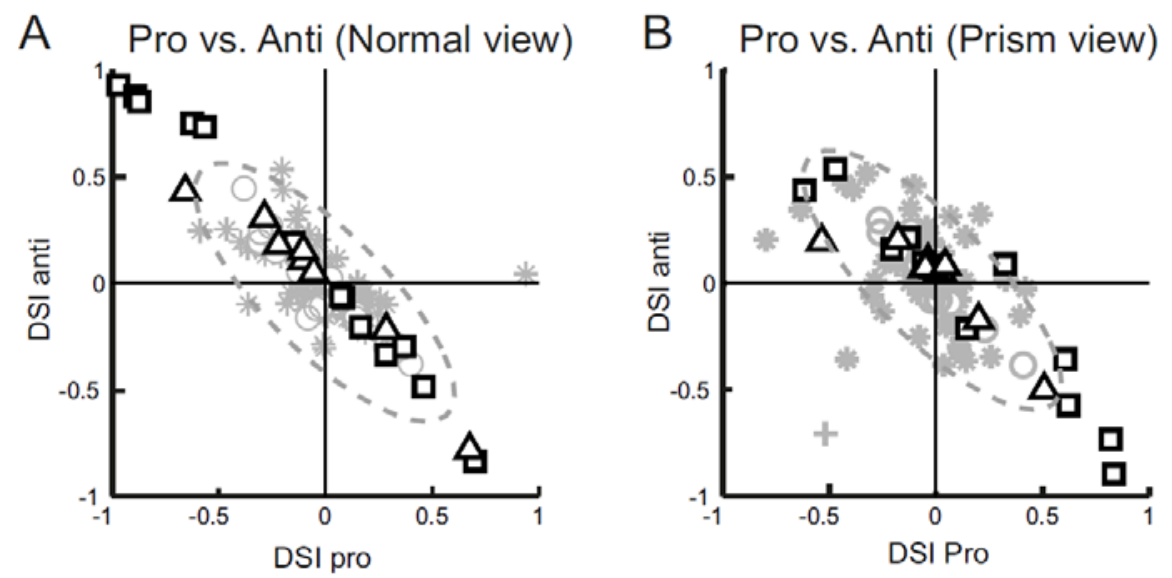

1st level classification (all neurons)

\section{Unclassifiable \\ $+\quad$ Visual memory \\ $\circ \mathbf{\square} \Delta$ Motor goal}

C Normal vs. Prism (Pro rule)
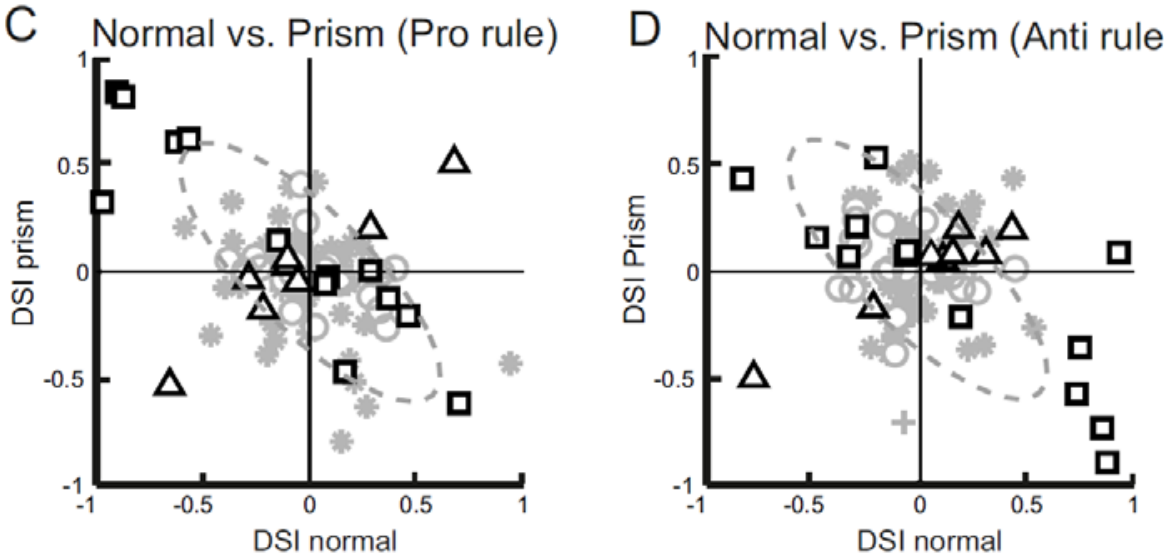

2nd level classification (motor goal neurons)

Undefined
$\square$ PG neuron
$\Delta$ VG neuron


Figure 4
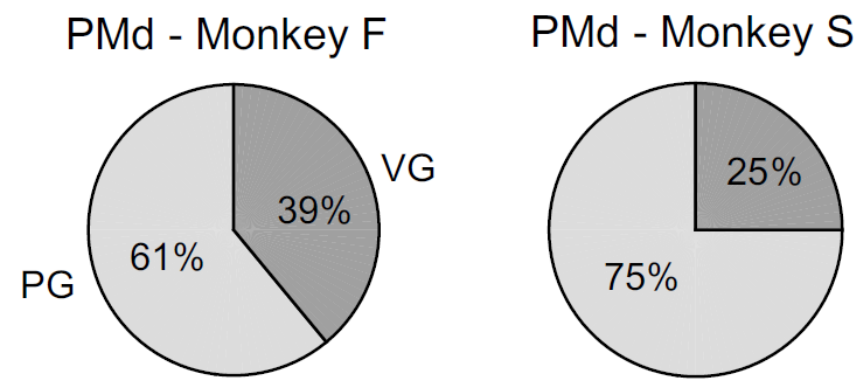

PMd - Monkey S (Combined task)
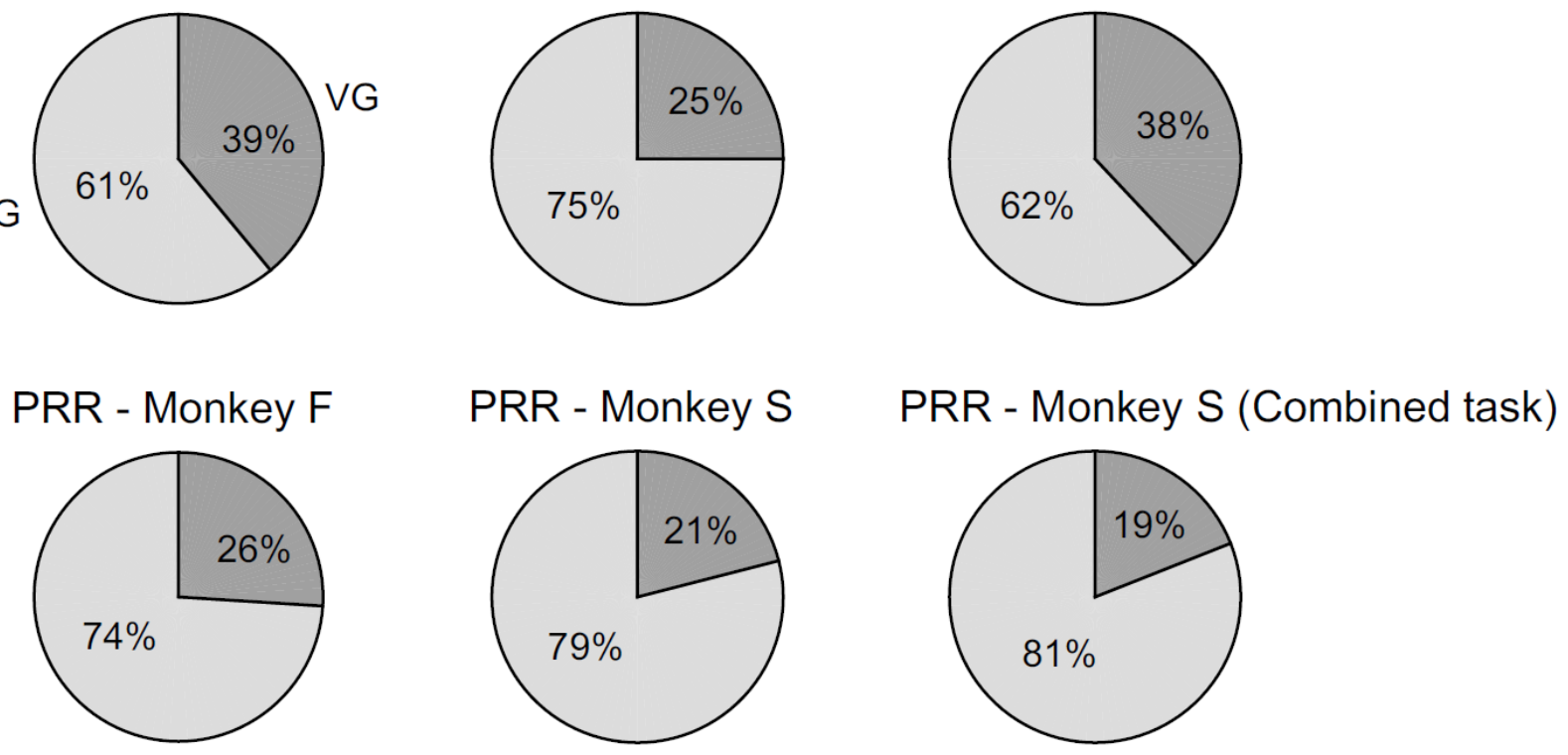

Figure 5
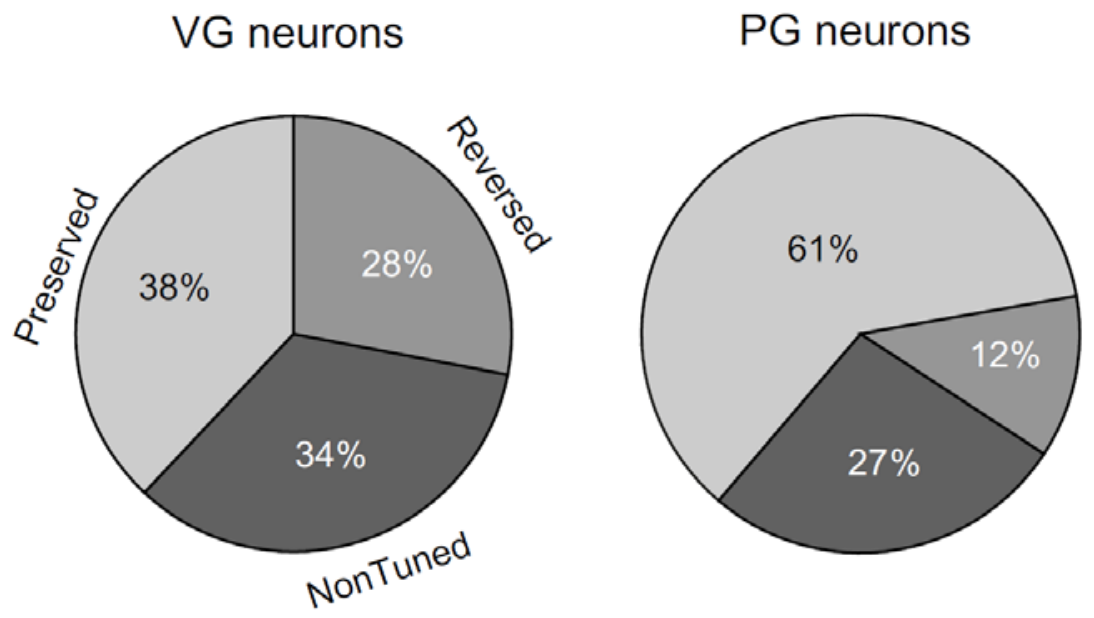
Figure 6

A

Monkey S - PMd
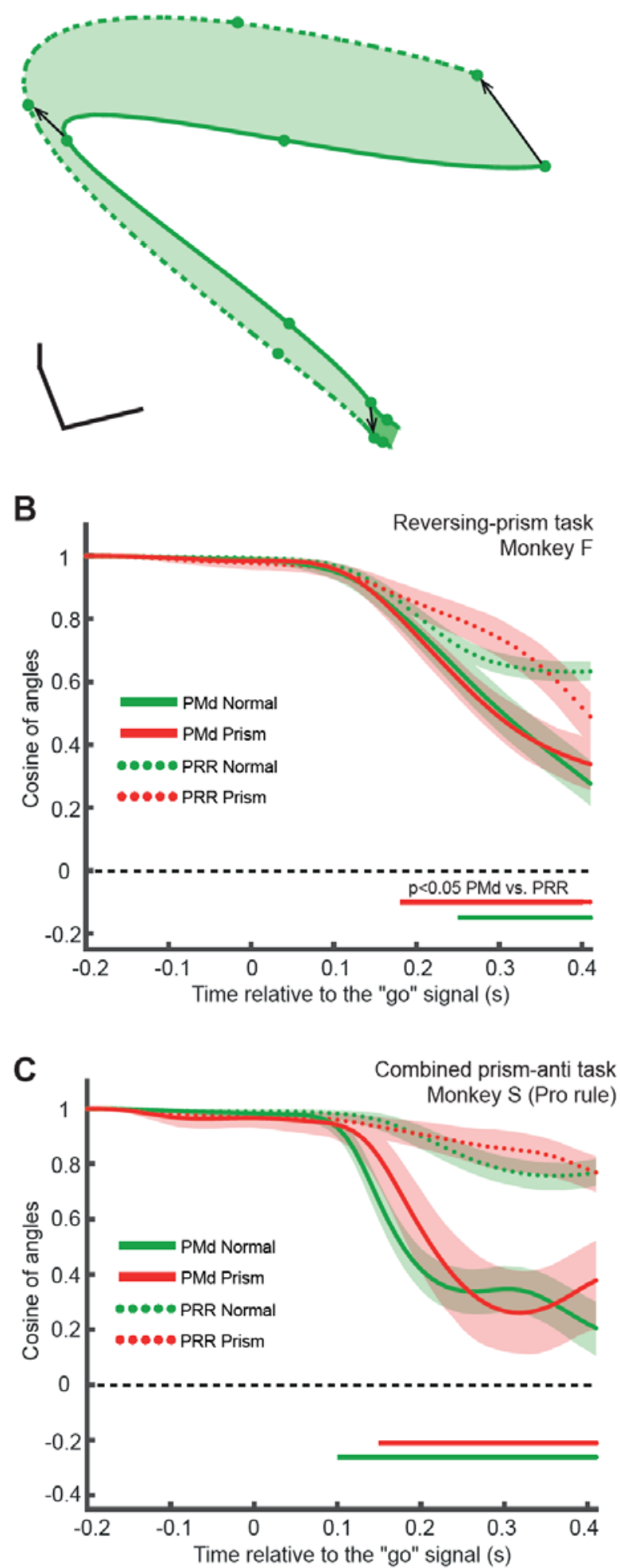

Monkey S - PRR
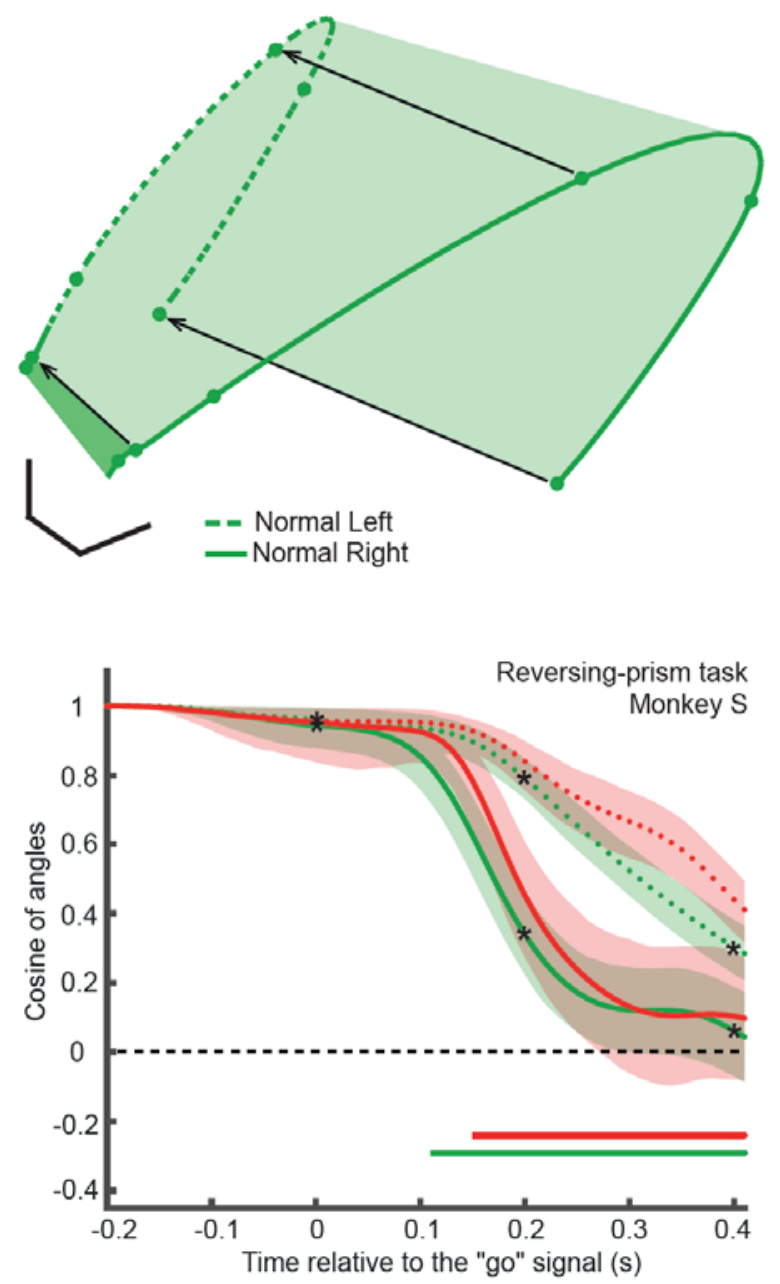

Combined prism-anti task Monkey S (Anti rule)

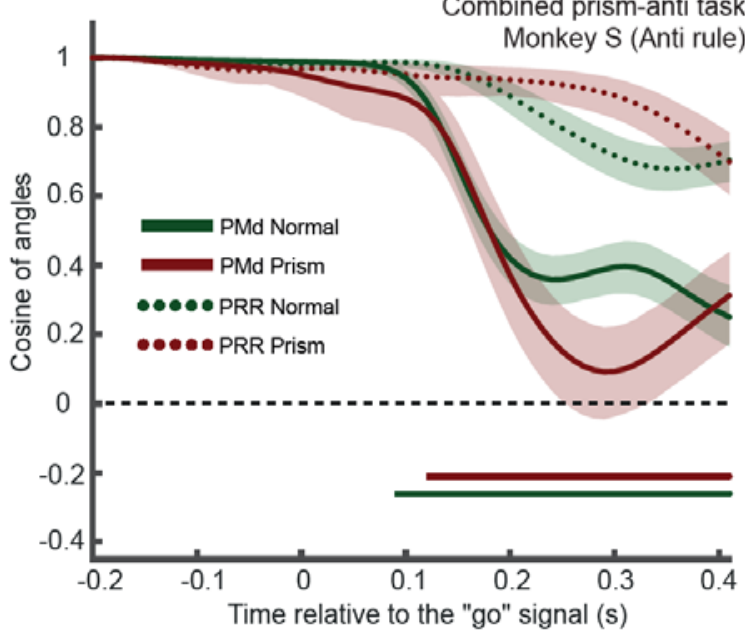


Table 1

Neuron categorization based on the delay period activity in each brain area for each monkey. Area PMd and PRR have similar percentages of PG and VG neurons.

\begin{tabular}{|l|c|c|c|c|}
\hline \multirow{2}{*}{$\begin{array}{c}\text { Neuron } \\
\text { Categorization }\end{array}$} & \multicolumn{2}{|c|}{ PMd } & \multicolumn{2}{c|}{ PRR } \\
\cline { 2 - 5 } & Monkey F & Monkey S & Monkey F & Monkey S \\
\hline Total recorded & $116(100 \%)$ & $140(100 \%)$ & $199(100 \%)$ & $163(100 \%)$ \\
\hline Not tuned & $16(14 \%)$ & $42(30 \%)$ & $48(24 \%)$ & $44(27 \%)$ \\
\hline Normal only & $29(25 \%)$ & $44(31 \%)$ & $65(32 \%)$ & $46(28 \%)$ \\
\hline Prism only & $25(21 \%)$ & $26(19 \%)$ & $33(17 \%)$ & $26(16 \%)$ \\
\hline Normal \& Prism & $46(40 \%)$ & $28(20 \%)$ & $53(27 \%)$ & $47(29 \%)$ \\
\hline & & & & $37(79 \%)$ \\
\hline PG neuron & $28(61 \%)$ & $21(75 \%)$ & $39(74 \%)$ & $10(21 \%)$ \\
\hline VG neuron & $18(39 \%)$ & $7(25 \%)$ & $14(26 \%)$ & 10 \\
\hline
\end{tabular}

Table 2

Frequency analysis of directionality from delay to reach epochs for each viewing context, each area and each monkey. We restricted our analysis to neurons that are selective in the delay epoch. We categorize these neurons according to their selectivity in the reach epoch. Note that the two datasets from monkey S were pooled.

\begin{tabular}{|l|c|c|c|c|c|c|c|c|}
\hline \multirow{2}{*}{$\begin{array}{l}\text { Directionality } \\
\text { from delay to } \\
\text { reach epoch }\end{array}$} & \multicolumn{4}{|c|}{ PMd } & \multicolumn{4}{c|}{ PRR } \\
\cline { 2 - 9 } & Nonkey F & \multicolumn{2}{|c|}{ Monkey S } & \multicolumn{2}{|c|}{ Monkey F } & \multicolumn{2}{c|}{ Monkey S } \\
\cline { 2 - 9 } & Prism & Normal & Prism & Normal & Prism & Normal & Prism \\
\hline Preserved & $32(43 \%)$ & $36(51 \%)$ & $65(34 \%)$ & $39(28 \%)$ & $72(61 \%)$ & $45(52 \%)$ & $128(62 \%)$ & $77(53 \%)$ \\
\hline Not-tuned & $28(37 \%)$ & $24(34 \%)$ & $80(42 \%)$ & $74(54 \%)$ & $22(19 \%)$ & $33(39 \%)$ & $46(22 \%)$ & $52(36 \%)$ \\
\hline Reversed & $15(20 \%)$ & $11(15 \%)$ & $45(24 \%)$ & $24(18 \%)$ & $24(20 \%)$ & $8(9 \%)$ & $32(16 \%)$ & $16(11 \%)$ \\
\hline Total & $75(100 \%)$ & $71(100 \%)$ & $190(100 \%)$ & $137(100 \%)$ & $118(100 \%)$ & $86(100 \%)$ & $206(100 \%)$ & $145(100 \%)$ \\
\hline
\end{tabular}




\section{References}

Andersen RA, Buneo CA (2002) Intentional maps in posterior parietal cortex. AnnuRev Neurosci 25:189-220.

Andersen RA, Cui H (2009) Intention, action planning, and decision making in parietal-frontal circuits. Neuron 63:568-583.

Archambault PS, Ferrari-Toniolo S, Battaglia-Mayer A (2011) Online control of hand trajectory and evolution of motor intention in the parietofrontal system. The Journal of neuroscience : the official journal of the Society for Neuroscience 31:742-752.

Bakker R, Wachtler T, Diesmann M (2012) CoCoMac 2.0 and the future of tracttracing databases. Frontiers in neuroinformatics 6:30.

Batista AP, Buneo CA, Snyder LH, Andersen RA (1999) Reach plans in eyecentered coordinates. Science 285:257-260.

Battaglia-Mayer A, Caminiti R, Lacquaniti F, Zago M (2003) Multiple levels of representation of reaching in the parieto-frontal network. Cereb Cortex 13:10091022.

Bosco A, Breveglieri R, Chinellato E, Galletti C, Fattori P (2010) Reaching activity in the medial posterior parietal cortex of monkeys is modulated by visual feedback. Journal of Neuroscience 30:14773-14785.

Bremner LR, Andersen RA (2014) Temporal analysis of reference frames in parietal cortex area $5 d$ during reach planning. The Journal of neuroscience : the official journal of the Society for Neuroscience 34:5273-5284.

Buneo CA, Jarvis MR, Batista AP, Andersen RA (2002) Direct visuomotor transformations for reaching. Nature 416:632-636.

Buneo CA, Batista AP, Jarvis MR, Andersen RA (2008) Time-invariant reference frames for parietal reach activity. Experimental brain research 188:77-89.

Caminiti R, Genovesio A, Marconi B, Mayer AB, Onorati P, Ferraina S, Mitsuda T, Giannetti S, Squatrito S, Maioli MG, Molinari M (1999) Early coding of reaching: frontal and parietal association connections of parieto-occipital cortex. Eur J Neurosci 11:3339-3345.

Chakrabarti S, Martinez-Vazquez P, Gail A (2014) Synchronization patterns suggest different functional organization in parietal reach region and dorsal premotor cortex. Journal of neurophysiology 112:3138-3153. 
Chang SW, Snyder LH (2010) Idiosyncratic and systematic aspects of spatial representations in the macaque parietal cortex. ProcNatlAcadSciUSA 107:79517956.

Churchland MM, Shenoy KV (2007) Temporal complexity and heterogeneity of single-neuron activity in premotor and motor cortex. Journal of Neurophysiology 97:4235-4257.

Churchland MM, Cunningham JP, Kaufman MT, Ryu SI, Shenoy KV (2010) Cortical preparatory activity: representation of movement or first cog in a dynamical machine? Neuron 68:387-400.

Churchland MM, Cunningham JP, Kaufman MT, Foster JD, Nuyujukian P, Ryu SI, Shenoy KV (2012) Neural population dynamics during reaching. Nature 487:51-56.

Crammond DJ, Kalaska JF (1994) Modulation of preparatory neuronal activity in dorsal premotor cortex due to stimulus-response compatibility. Journal of neurophysiology 71:1281-1284.

Crawford JD, Henriques DY, Medendorp WP (2011) Three-dimensional transformations for goal-directed action. Annual Review of Neuroscience 34:309331.

Desmurget M, Sirigu A (2009) A parietal-premotor network for movement intention and motor awareness. Trends Cogn Sci 13:411-419.

Desmurget M, Reilly KT, Richard N, Szathmari A, Mottolese C, Sirigu A (2009) Movement intention after parietal cortex stimulation in humans. Science 324:811813.

Duhamel JR, Colby CL, Goldberg ME (1992) The updating of the representation of visual space in parietal cortex by intended eye movements. Science 255:90-92.

Elsayed GF, Lara AH, Kaufman MT, Churchland MM, Cunningham JP (2016) Reorganization between preparatory and movement population responses in motor cortex. Nature communications 7:13239.

Franklin DW, Wolpert DM (2011) Computational mechanisms of sensorimotor control. Neuron 72:425-442.

Gail A, Andersen RA (2006) Neural Dynamics in Monkey Parietal Reach Region Reflect Context-Specific Sensorimotor Transformations. Journal of Neuroscience 26:9376-9384.

Gail A, Klaes C, Westendorff S (2009) Implementation of spatial transformation rules for goal-directed reaching via gain modulation in monkey parietal and premotor cortex. Journal of Neuroscience 29:9490-9499. 
Hawkins KM, Sayegh P, Yan X, Crawford JD, Sergio LE (2013) Neural activity in superior parietal cortex during rule-based visual-motor transformations. Journal of cognitive neuroscience 25:436-454.

Hommel B, Musseler J, Aschersleben G, Prinz W (2001) The Theory of Event Coding (TEC): a framework for perception and action planning. The Behavioral and brain sciences 24:849-878; discussion 878-937.

Jeannerod M (2001) Neural simulation of action: a unifying mechanism for motor cognition. NeuroImage 14:S103-109.

Johnson PB, Ferraina S, Bianchi L, Caminiti R (1996) Cortical networks for visual reaching: physiological and anatomical organization of frontal and parietal lobe arm regions. Cerebral cortex (New York, NY : 1991) 6:102-119.

Kalaska JF (1996) Parietal cortex area 5 and visuomotor behavior. CanJPhysiol Pharmacol 74:483-498.

Kalaska JF, Scott SH, Cisek P, Sergio LE (1997) Cortical control of reaching movements. Current opinion in neurobiology 7:849-859.

Kaufman MT, Churchland MM, Ryu SI, Shenoy KV (2014) Cortical activity in the null space: permitting preparation without movement. Nat Neurosci 17:440-448.

Klaes C, Westendorff S, Chakrabarti S, Gail A (2011) Choosing goals, not rules: deciding among rule-based action plans. Neuron 70:536-548.

Kuang S, Morel P, Gail A (2016) Planning Movements in Visual and Physical Space in Monkey Posterior Parietal Cortex. Cereb Cortex 26:731-747.

Lehmann SJ, Scherberger H (2013) Reach and gaze representations in macaque parietal and premotor grasp areas. The Journal of neuroscience : the official journal of the Society for Neuroscience 33:7038-7049.

Luppino G, Calzavara R, Rozzi S, Matelli M (2001) Projections from the superior temporal sulcus to the agranular frontal cortex in the macaque. Eur $\mathrm{J}$ Neurosci 14:1035-1040.

Marconi B, Genovesio A, Battaglia-Mayer A, Ferraina S, Squatrito S, Molinari M, Lacquaniti F, Caminiti R (2001) Eye-hand coordination during reaching. I. Anatomical relationships between parietal and frontal cortex. Cerebral cortex (New York, NY : 1991) 11:513-527.

McGuire LM, Sabes PN (2011) Heterogeneous representations in the superior parietal lobule are common across reaches to visual and proprioceptive targets. Journal of Neuroscience 31:6661-6673. 
Michaels JA, Dann B, Scherberger H (2016) Neural Population Dynamics during Reaching Are Better Explained by a Dynamical System than Representational Tuning. PLoS computational biology 12:e1005175.

Mulliken GH, Musallam S, Andersen RA (2008) Forward estimation of movement state in posterior parietal cortex. ProcNatlAcadSciUSA 105:8170-8177.

Ochiai T, Mushiake H, Tanji J (2002) Effects of image motion in the dorsal premotor cortex during planning of an arm movement. Journal of Neurophysiology 88:2167-2171.

Ochiai T, Mushiake H, Tanji J (2005) Involvement of the ventral premotor cortex in controlling image motion of the hand during performance of a target-capturing task. Cerebral Cortex 15:929-937.

Pesaran B, Nelson MJ, Andersen RA (2006) Dorsal premotor neurons encode the relative position of the hand, eye, and goal during reach planning. Neuron 51:125134.

Pesaran B, Nelson MJ, Andersen RA (2008) Free choice activates a decision circuit between frontal and parietal cortex. Nature 453:406-409.

Schwartz AB, Moran DW, Reina GA (2004) Differential representation of perception and action in the frontal cortex. Science 303:380-383.

Scott SH (2004) Optimal feedback control and the neural basis of volitional motor control. Nature reviews Neuroscience 5:532-546.

Shadmehr R, Smith MA, Krakauer JW (2010) Error correction, sensory prediction, and adaptation in motor control. Annual Review of Neuroscience 33:89-108.

Shen L, Alexander GE (1997) Preferential representation of instructed target location versus limb trajectory in dorsal premotor area. Journal of Neurophysiology 77:1195-1212.

Shenoy KV, Sahani M, Churchland MM (2013) Cortical control of arm movements: a dynamical systems perspective. Annu Rev Neurosci 36:337-359.

Shin YK, Proctor RW, Capaldi EJ (2010) A review of contemporary ideomotor theory. Psychological bulletin 136:943-974.

Snyder LH, Batista AP, Andersen RA (1997) Coding of intention in the posterior parietal cortex. Nature 386:167-170.

Waszak F, Cardoso-Leite P, Hughes G (2012) Action effect anticipation: neurophysiological basis and functional consequences. Neuroscience and biobehavioral reviews 36:943-959. 
Westendorff S, Klaes C, Gail A (2010) The cortical timeline for deciding on reach motor goals. Journal of Neuroscience 30:5426-5436.

Wise SP, Boussaoud D, Johnson PB, Caminiti R (1997) Premotor and parietal cortex: corticocortical connectivity and combinatorial computations. Annual Review of Neuroscience 20:25-42. 


\section{2 - Reconfiguration of population dynamics for context-dependent sensorimotor transformations}

In goal-directed reach movement, the same visual stimulus can lead to very different decisions and actions, depending on specific behavioral contexts. Context-dependent sensorimotor transformation is essential for selection among alternative actions. So far, the underlying computational strategies that support flexible sensorimotor transformation remain unsolved.

We examined the possible population-level computational strategies in macaque PMd and PRR during the preparatory stage of center-out reaching tasks with two different contextual configurations. In normal-/prism-reach task, the contextual information was introduced into the neural system by applying a reversed-viewing prism (prism-reach). In pro-/anti-reach task, the contextual information was introduced through arbitrary transformation rule (anti-reach). We found that there exist non-overlapping population-level subspaces dedicated to the visuomotor transformations in normal- and prism-trials. In contrast, anti-trials exploit overlapped subspace as the pro-trials. In addition, we identified a systematically shifted baseline neural activity exclusively in the prism viewing context. These results provide direct evidence for the notion that specific brain area employs distinguishable neural computations in different context-dependent sensorimotor transformations. 


\title{
Reconfiguration of population dynamics for context-dependent sensorimotor transformations
}

\author{
Hao Guo ${ }^{1,2}$, Shenbing Kuang ${ }^{3}$, Alexander Gail ${ }^{1,2,4}$ \\ ${ }^{1}$ German Primate Center, Göttingen, Germany \\ ${ }^{2}$ Georg-August-Universität Göttingen, Göttingen, Germany \\ ${ }^{3}$ Institute of Psychology, Chinese Academy of Sciences, Beijing, China \\ ${ }^{4}$ Bernstein Center for Computational Neuroscience, Göttingen, Germany
}

\begin{abstract}
Context-dependent sensorimotor transformation is essential for flexible selection among alternative actions. In real life situation, the motor goal is often inferred from the location of sensory stimulus based on contextual information. Although such space-context integration is typical, the underlying computational strategies that support flexible sensorimotor transformation remain to be elaborated. Neural computations in a dynamical system can be configured by controlling the system's intrinsic dynamics, inputs and initial conditions. To investigate whether the brain exploits such mechanisms, we examined population responses in macaque dorsal premotor cortex (PMd) and parietal reach region (PRR) in two context-dependent center-out reaching tasks. The contextual information was introduced into the neural system either by applying reversed-viewing prism (prism-reach) or through transformation rule (anti-reach). We found that there exist non-overlapping population-level subspaces dedicated to the visuomotor transformations in normaland prism-trials. In contrast, the transformations in anti-trials exploit overlapped subspace as pro-trials. Thus, computational strategies for space-context integration
\end{abstract}


differ under distinct behavioral contexts, proving the contextual information could configure cortical computation by adjusting the system's intrinsic dynamics. Besides, the baseline activity in neural state space under the prism viewing context is consistent with the mechanism wherein the contextual information affects cortical dynamics by systematically shifting the initial conditions in state space. We further defined a dimension which discriminates the block-designed normaland prism-reaches based on their baseline activities. This initial condition-related dimension is orthogonal to the dimensions that encode motor goals. Compare to the anti-reach in which pure spatial remapping is required, the prism-reach needs different sensorimotor plants for motor control because of the misaligned visual and proprioceptive feedback. The influence of contextual information was either instant (anti-reach) or predictable (prism-reach), leading to different computational mechanisms for sensorimotor transformations.

\section{Introduction}

Goal-directed movement includes flexible selection among alternative actions depending on the behavioral context. The same sensory stimulus can lead to very different decisions and actions, depending on the current behavioral context. Such space-context integration in goal-directed reaching has been associated with the frontoparietal reach network in the cerebral cortex, but the underlying computational strategies that support flexible sensorimotor transformation remain unsolved. Previous studies investigated the single-neuron activity in frontoparietal reach network, and revealed that the neural activity varies under distinct behavioral contexts (Wallis and Miller, 2003; Gail and Andersen, 2006; Pesaran et al., 2008; Gail et al., 2009; Westendorff et al., 2010; Klaes et al., 2011; Kuang et al., 2016). 
Nevertheless, largely heterogeneous and time-varying single-neuron activities obscure the neural dynamics of specific neural computation. From a dynamical system perspective, the covariance pattern across the neural population is the signature of unique neural computation, and the way neurons co-vary with each other is believed to be constrained by the underlying network connectivity (Sadtler et al., 2014; Gallego et al., 2017). Thus, the complex single-neuron activity can be described in a dynamical system for systematic explanations (Shenoy et al., 2013; Cunningham and Yu, 2014). Here we investigated how the neurons, either from dorsal premotor cortex (PMd) in the frontal lobe (Wise et al., 1997) or from parietal reach region (PRR) in the posterior parietal cortex (Snyder et al., 1997), systematically configure their population activity to accomplish context-dependent sensorimotor transformations.

We examined the possible population-level computational strategies in macaque PMd and PRR during the preparatory stage of instructed-delay center-out reaching tasks with two different contextual configurations. Both tasks required contextdependent selection and integration of visual stimuli. In the prism-reach (Fig. 1A), monkeys were trained to plan reaches under either normal or prism-reversed viewing conditions (Kuang et al., 2016). In the prism context with, for example, a perceived right-side visual cue, the monkeys would need to physically reach to the left in order to visually bring the hand toward the memorized visual cue location. In anti-reach (Fig. 1B), monkeys were trained to plan reaches based on learned visuomotor association (Westendorff et al., 2010). Context-specific transformation rules either instructed a reach toward the visual cue (rule pro) or its opposite location (rule anti). This rule-based reach task has been applied to answer the question of whether PMd and PRR neurons represent the memorized location of the visual cue (retrospective) or the pending movement goal (prospective) during 
reach planning (Gail and Andersen, 2006; Gail et al., 2009). In both reach tasks, spatial representation of a visual cue had to be remapped onto a spatially opposite motor goal during the visuomotor transformation, and the motor outputs of these two tasks were physically equivalent given identical visual cues. Visuomotor transformation involves the temporal evolution of the information processing from visual stimuli to reaching movement. While sensory stimulus provides information about the possible object and evokes a reaching aiming at it, neural activity reflects the "default" movement plan in this neutral background; we referred to this activity as the default computation, because a spatial remapping based on contextual information is not required. In context-dependent sensorimotor transformation (prism- and anti-reach), the reach planning demanded an integration between sensory stimulus and contextual information; we referred to the neural activity as the context-specific computation. Interest in independently studying two related computations that are both implemented in the same neural population has recently increased, leading to questions such as how the same neural population subserves both default and context-specific computations.

The same neural population is able to perform different computations depending on the behavioral demands (Raposo et al., 2014; Elsayed et al., 2016). The brain could recruit different covariance patterns based on the computations being performed (Gallego et al., 2017). While describing the population activity in a high-dimensional state space, the covariance patterns in a typical experiential setting are often confined to a low-dimensional subspace (Gallego et al., 2017) that is called neural manifold (Fig. 1C). In recent studies, a dynamical systems view has been used to describe neural manifold and neural trajectories in prefrontal, premotor and parietal cortical areas in various cognitive tasks (Machens et al., 2010; Mante et al., 2013; Hennequin et al., 2014; Kaufman et al., 2014; Raposo et 
al., 2014; Sadtler et al., 2014; Elsayed et al., 2016; Gallego et al., 2017; Wang et al., 2018). These studies emphasized the important role of neural population dynamics in understanding how cortical activity patterns support flexible computations. From the dynamical system perspective, population activity in any specific area can be described in terms of three factors: (1) the system's intrinsic dynamics which is defined by the covariation between interconnected neurons, (2) the external inputs, and (3) the initial state of a system. These concepts have led to our hypothesis that the space-context integration in sensorimotor transformation might be approached in different ways which are corresponding to these three components. Thus, we explored the possible population-level strategies which are related to intrinsic dynamics, the external inputs and initial state separately. The first type of population-level strategy arises when contextual information exerts influence on the intrinsic dynamics by driving the population activity in contextspecific computation out of the neural manifold defined by default computation (Fig. 1C). In this scenario, neural activities during the two computations are independent on the population level. The second strategy arises when contextual information enters the cortical network as an external input without changing the intrinsic dynamics, the neural activity could follow a trajectory within the manifold. Thus, neural activity during default computation and context-specific computation exploit the same neural manifold (Fig. 1D). The third strategy associates different contextual information with separated initial states, from which the neural population response should evolve predictably.

Here we report that the specific brain area employs distinguishable neural computations in different context-dependent sensorimotor transformations. In the prism-reach task, contextual information affects the intrinsic dynamics, leading to non-overlapping population-level subspaces dedicated to normal- and prism-trials. 
In contrast, anti-trials exploit similar subspace as pro-trials. In addition, we identified a systematically shifted baseline neural activity in PRR as a robust correlate of prism viewing context.

\section{Materials and Methods}

The technical details of the behavioral apparatus and experimental procedures were described previously (Westendorff et al., 2010; Kuang et al., 2016). All experimental procedures were conducted in accordance with institutional guidelines on Animal Care and Use of the German Primate Center, the European Directive 2010/63/EU, the corresponding German national law and regulations governing animal welfare, and were approved by regional authorities where necessary.

\section{Prism-reach task}

Two monkeys were trained in a memory-guided center-out reaches under either a normal or a prism viewing context (Kuang et al., 2016). There were only two possible visual cue locations either to the left or to the right of the central fixation spots, at constant positions over all experimental sessions. In the prism context with a perceived right-side visual cue, for example, the monkeys would need to physically reach to the left in order to visually bring the hand toward the memorized visual cue location (prism condition, upper right panel in Fig. 1A). Normal and prism trials were alternated in blocks of 40 trials by manually switching between the prism and the empty box in the aperture. Most recording sessions had four blocks, with two blocks in each viewing context. 
Each trial started with a variable-length fixation period (0.75-1.25 s), followed by $0.2 \mathrm{~s}$ of visual cue presentation, and then a 1-2 s variable delay period during which the visual cues were absent (delay period). Center-out reaches were made to peripheral targets with an eccentricity of $5 \mathrm{~cm}\left(7.1^{\circ}\right.$ visual angle, tolerance of $\left.2 \mathrm{~cm}\right)$ in response to the disappearance of the central hand fixation spot (go signal). During these time periods, the monkeys had to keep both eye and hand fixation at the center of the screen (tolerance of $2.9^{\circ}$ of visual angle). The monkeys received liquid reward for correct trials. Fingertip movements were continuously optically tracked to rule out on-line movement reversals.

\section{Anti-reach task}

Two monkeys were trained in a memory-guided center-out anti-reach task (Westendorff et al., 2010). The anti-reach task required the subjects to map a spatial cue onto one of two motor goals, either at the location of the spatial cue (pro-reach) or opposite to it (anti-reach). The four peripheral spatial cue positions (right, $0^{\circ}$; up, $90^{\circ}$; left, $180^{\circ}$; down, $270^{\circ}$ direction) were centered around the central fixation point at $9 \mathrm{~cm}$ eccentricity. The contextual cue (colored frame around the central eye and hand fixation points) instructed the subject to reach toward (pro-reach; green cue) or diametrically opposite (anti-reach; blue cue) of the spatial cue. The eight task conditions (two context conditions $\times$ four cue directions) were pseudo-randomly interleaved from trial to trial.

The timeline of the trials was as follows (Fig. 1B): The monkey initiated a trial by acquiring central eye fixation (tolerance of $2.5-4.0^{\circ}$ of visual angle; CCD camera, Thomas Recording) and hand fixation at a touch screen. A variable-length fixation period (0.5-1 s) was followed by the brief visual cue period (0.2 s). The peripheral spatial cue and contextual cue were flashed simultaneously. For a variable 
duration, the monkey had to keep both eye and hand fixation (memory period, 0.8$2 \mathrm{~s}$ ). When the central hand fixation target turned off (go signal), the monkey had to reach (movement period, maximum of $0.7-1 \mathrm{~s}$ ) toward the instructed goal. The monkey received visual feedback about the correct movement goal (circular patch stimulus at the motor goal location) when he acquired the desired position. The monkey had to keep his hand at the reach target location (0.3-0.4 s) to successfully finish the trial and receive liquid reward. Ocular fixation had to be kept throughout the course of the trial.

\section{Animal preparation}

Two custom-fit recording chambers were implanted to each monkey's skull contralateral to the handedness of the monkeys. The implantation of each chamber, one for PRR and the other for PMd, was guided by pre-surgical MRI and confirmed by post-surgical MRI. All imaging and surgical procedures were conducted under general anesthesia.

\section{Neural data acquisition}

After the monkeys became proficient in the tasks (prism-reach: monkeys $\mathrm{S}$ and $\mathrm{F}$; anti-reach: A and S), neural activity of PRR and PMd were recorded simultaneously with multiple electrodes in each area in each session. The $x-y$ electrode locations within the chamber were positioned in each recording session using the xyz-manipulator (mini-Matrix, Thomas Recording) that holds the microdrive with sub-millimeter resolution. The chamber coordinates relative to cortex were extracted from post-surgical MRI, allowing navigation and positioning of penetration sites relative to anatomical landmarks. For all neuronal spiking data, spikes were sorted offline (Offline Sorter; Plexon). All well-isolated task- 
responsive neurons were recorded and no attempt was made to screen for neuronal tuning for reach direction or any other response property.

\section{Data preprocessing}

In order to yield highly reliable trial-averaged neural responses that capture both the temporal dynamics and the relationships among the task variables, we only picked the neurons from which at least 10 repeated trials were recorded for each condition. 255 neurons in PMd (monkey S, 139; monkey F, 116) and 359 neurons in PRR (monkey S, 162; monkey F, 197) were involved in prism-reach task analyses. 153 neurons in PMd (monkey A, 100; monkey S, 53) and 165 neurons in PRR (monkey A, 115; monkey S, 50) were involved in anti-reach task analyses. For analyses based on principal component analysis (PCA), subspace identification and Euclidean distance calculation, we applied the following pre-processing steps. First, the spikes were smoothed across time with a Gaussian kernel with standard deviation (s.d.) of $20 \mathrm{~ms}$ and averaged across trials to produce peri-stimulus time histograms (PSTH). The neural responses were sampled every $10 \mathrm{~ms}$. Neural responses for each neuron were then mean-centered at each time as follows: we calculated the mean activity across all conditions of each neuron at each time point and subtracted this mean activity from each condition's response (to avoid bias toward high firing rate neurons). All data were aligned at visual cue onset. For analyses based on neural state space, neurons that were not recorded simultaneously were combined as pseudo-simultaneous population activity patterns. Population-level activity is defined in a high-dimensional neural state space in which each dimension represents the activity of one recorded neuron. We grouped the trial-averaged neural activity in normal-/prism-reach data into the matrix $P \in \mathbb{R}^{N \times C T}$, where $\mathrm{N}$ is the total number of neurons, $C$ is the number of conditions (4 conditions in data: 2 directions $\times 2$ viewing contexts) and $T$ is the number of 
time points (all times within the visuomotor transformation epoch, 400 ms aligned to visual cue onset). Similarly, we grouped the neural responses in the pro/antireach data into the matrix $A \in \mathbb{R}^{N \times C T}$. $C$ is the number of conditions (4 conditions in data: 2 directions $\times 2$ rule cues). A low-dimensional subspace embedded within the high-dimensional neural state space was then estimated by using PCA on either matrix $P$ or $A$, the dimensionality (number of rows) was reduced to 10 . This dimensionality was estimated from the data, and the results were not sensitive to the exact dimensionality we used.

\section{Variance alignment analysis}

We initially reduced the dimensionality of the data as above to $\mathrm{k}$ dimensions (chosen as 10) using PCA. For this analysis, the matrix $P$ on which we performed PCA contained data from both the normal- $\left(P_{\text {normal }} \in \mathbb{R}^{k \times c T}, c=2\right)$ and prism( $P_{\text {prism }} \in \mathbb{R}^{k \times c T}, c=2$ ) condition together; this ensured that the resulting space captured the structure of both conditions. We then applied PCA on the $P_{\text {normal }}$ alone so that the rotated k-dimensional spaces only captured as much $P_{\text {normal }}$ data variance as possible, all components were retained. The $P_{\text {prism }}$ were then rotated into this normal-condition-determined orientation. For each dimension d (1 to k, horizontal axis in Fig. 2), we could then determine how much variance was present in the first $\mathrm{d}$ dimensions of the $P_{\text {prism }}$. These values were normalized by the maximum possible variance that could be captured in the same number of dimensions (that is, if the rotation were found using PCA on the $P_{\text {prism }}$ itself). Perfect alignment would produce a unity variance alignment value, while maximal misalignment defines the lower bound (that is, if the highest variance dimension in $P_{\text {normal }}$ were the lowest variance dimension in $\left.P_{\text {prism }}\right)$. To determine the chance variance alignment, 1,000 randomly oriented orthogonal bases for the k-space were 
chosen. The 95\% confidence interval (Figure 2) was then defined as the interval spanning from the 2.5th to the 97.5th percentile of the resampled values derived from these random bases. The variance alignment analysis applied onto matrix $A$ was similar, except for using $A_{\text {pro }}, A_{\text {anti }} \in \mathbb{R}^{k \times c T}(c=2)$ instead.

\section{Euclidean distance analysis}

Population-level neural differences between different conditions were quantified using a firing rate Euclidian distance measurement. When applying, for example, onto matrix $P \in \mathbb{R}^{N \times C T}$, we separately quantified the time-varying firing rate difference between left and right trials in either normal or prism viewing context. The population activity for each condition could be described as a $\mathbb{R}^{N \times T}$ matrix. We then subtracted the left and right matrices element by element in either viewing context, resulting in a single $D^{N \times T}$ matrix of firing rate differences for each time point and unit. To convert the $D^{N \times T}$ to a time-resolved neural population distance measure, we took the vector 2-norm of the $t$-th column of matrix $D^{N \times T}$. Because a vector norm is by definition non-negative, there always will be some firing rate distance between any two different trials due to single-trial spiking variability even if there were no differences in the firing rate. We therefore used a bootstrap procedure to calculate what this distance would be if the null hypothesis is that the two groups (e.g., left-trials vs. right-trials) came from the same distribution. We generated 1000 shuffled datasets where trials' left and right labels were shuffled randomly. The Euclidean distance was then computed between these faux-left and faux-right conditions, resulting in 1000 shuffled Euclidean distances. For each time point, we subtracted the mean distance across the corresponding shuffled distances from the data's distance. If the result value was larger than 0, then the Euclidean distance was greater than what is expected by chance. These shuffled distances were also used to perform a nonparametric test for significance: if all of the 
shuffled distances at a time point were smaller than the data distance, the Euclidean distance at this given time point was statistically significant at $\mathrm{p}<0.001$.

\section{Identifying motor goal encoding dimensions}

We developed a method that identified the optimal dimensions of the data on which motor goals representations are maximally separated in both normal and prism viewing contexts (encoding dimension). We defined the vector of selectivity (VOS) which discriminates directions (that is, right- or left-reach) in either viewing context, and activity projected along the VOS contains almost all directionselective activity. We defined VOS in normal and prism viewing context as $\overrightarrow{v_{n}}$ and $\overrightarrow{v_{p}}, \in \mathbb{R}^{N \times T}$ ( $T$ is the number of time points during late memory period, that is, 300 ms before go cue onset). We then found principal components $w \in \mathbb{R}^{k \times N}$ in neural space that could optimize the following objective:

$$
\max _{w} \operatorname{Tr}\left(w \overrightarrow{v_{n}}\left(w \overrightarrow{v_{n}}\right)^{T}+w \overrightarrow{v_{p}}\left(w \overrightarrow{v_{p}}\right)^{T}\right)
$$

which indicates maximizing the divergence between left and right conditions in both normal and prism viewing context. Thus, the optimal $w$ is given by the eigenvectors of the positive-definite matrix which is the sum of two $\mathbb{R}^{N \times N}$ correlation matrices. We call $w$ the encoding dimensions (PCs), because it represents the axis on which neural population encodes left- and right- motor goals. 


\section{Results}

\section{Behavioral Performance}

Two macaque monkeys were trained ( $\mathrm{F}$ and $\mathrm{S}$ ) to perform instructed-delay centerout reaching tasks under prism-reversed viewing context (Fig. 1A). Behavioral results and single-neuron analyses were reported before (Kuang et al., 2016). Both monkeys had become well acquainted with the normal and prism-reversed viewing contexts and performed the reaching task with high performance. The overall success rates, after subtracting trials with ocular or hand fixation breaks, belated responses, and erroneous choices, were 79\% for monkey $\mathrm{F}$ and $76.3 \%$ for monkey $\mathrm{S}$ in the normal viewing context and 79\% (F) and 78.5\% (S) in the prism viewing context. Most error trials were attributable to terminations early during the trial (ocular or hand fixation breaks before the go signal), rather than the confusion of the reach directions. The percentages of correct reach choices in non-aborted trials were 99.3\% (F) and 98.8\% (S) in the normal viewing context, and 99.2\% (F) and $98.6 \%$ (S) in the prism viewing context.

Two macaque monkeys were trained ( $\mathrm{S}$ and $\mathrm{A}$ ) to perform instructed-delay centerout pro-/anti- reaching tasks (Fig. 1B). Behavioral results and single-neuron analyses were reported before (Westendorff et al., 2010). Both monkeys performed the reaching task with high performance. The overall success rates were $77 \%$ for monkey $\mathrm{S}$ and $86 \%$ for monkey $\mathrm{A}$ in both pro- and anti-reaches. Errors were mainly caused by ocular fixation failures, not by incorrect target choices. The choice of reach target was correct in 97\% for monkey S, and in 99\% for monkey A. 


\section{Different computations occur in separate subspaces}

The datasets were collected from rhesus monkeys while they were performing goal-directed reach movement under distinct behavioral contexts. We applied dynamic system analyses to single neuronal spiking data recorded by extracellular recording in rhesus monkeys while they were performing blocked designed normal- and prism-reach task, or interleaved pro- and anti-reach task. A blockdesigned pro-/anti-reach task was used for the control test.

Neural state space could explore other dimensions (patterns of neural covariance) when the network needs to perform a substantially different function. The strategy has been proved to be widely present across the brain (Harvey et al., 2012; Mante et al., 2013; Kaufman et al., 2014; Raposo et al., 2014; Sadtler et al., 2014). We searched for a signature on the neural states either aligning or exploring independent dimensions during context-specific visuomotor transformation. We defined the visuomotor transformation epoch as a $400 \mathrm{~ms}$ interval beginning $50 \mathrm{~ms}$ before visual cue onset. In the prism-reach task, the population activity during the transformation epochs in normal- and prism-viewing context was described in the same high-dimensional state space. To quantify the alignment of normal- and prism-subspace, we used an analysis called variance alignment (see Materials and Methods). The analysis method was reported before (Raposo et al., 2014). This analysis method is based on the principal component analysis (PCA): the principal components are the eigenvectors of the covariance matrix of the neural activity. If neurons' firing rates co-vary in similar ways during these two visuomotor transformation epochs, then the normal-subspace principal components will capture much of the variance of the prism data, and the prism-subspace principal components will capture much of the variance for the normal data. Alternatively, if the PCs which define one subspace only account for little variance in the other data: 
that is, the covariance pattern is completely different from one data to the other. In the latter scenario, the two subspaces can be described as misaligned. It is noteworthy that this measure describes whether the neural state occupies the same dimensions (preserve the same covariance patterns), not whether the neural trajectories are similar within those dimensions.

In both monkeys, we found that the top PCs in the "default" movement planning under normal viewing context captured the variance in prism data far less well (Fig. 2A), supporting the hypothesis that the visuomotor transformations in default-trials and prism-trials exploit independent subspaces. To assess the baseline alignment, we randomly sampled oriented orthogonal bases with different dimensions (see Materials and Methods). We found the alignment variance is significantly less than chance level (95\% confidence interval; see Materials and Methods).

We applied the same analysis in anti-reach datasets when visuomotor transformations in pro- and anti-reaches were described in the same state space. The top PCs in "default" movement planning (pro-reach) captured a large amount of variance of anti-reach trials (Fig. 2B) in both monkeys. This result revealed a strong alignment between pro- and anti-subspaces, indicating the visuomotor transformation in anti-reaches exploits the same subspace as pro-reaches. Thus, PRR neurons explore significantly different covariance patterns of population activity in prism viewing context, but they employ similar covariance patterns in anti-reach trials.

We also repeated the analysis from Fig. 2 for PMd neural activity. The results with respect to subspace alignment were similar between PRR and PMd (Fig. 3). In both areas, normal-subspaces and prism-subspaces were more strongly misaligned than expected if they were random draws from the full-dimensional state space, while 
pro-subspaces and anti-subspaces were mainly overlapped with each other, which implies that the neural manifold is preserved across these two conditions.

\section{The neural activity in the prism viewing context starts from a different baseline state}

During movement planning, the neural responses always start from an initial condition, which is followed by evolving to a different final motor goal related states depending on the spatial stimulus and contextual information. In the prismreach task, normal and prism trials were alternated in blocks of 40 trials by manually switching between the prism and the empty box in the aperture (see Materials and Methods). Because of the block-designed task, the monkeys could visually identify the differences when there was a prism was putting in front of them. We asked if the neural population activity in both PRR and PMd reset their initial population response even before the visual cue onset. We first calculated the baseline neural activity on the single-neuron level, the distribution of all neuron's firing rate and the average firing rate across neurons were similar between normal and prism viewing context (Supplementary Fig. 1). We then calculated the timeresolved Euclidean distance between neural trajectories (see Materials and Methods).

In both normal and prism viewing context, neural trajectories that represent leftand right-side reach planning diverged in state space after visual cue onset (Fig. 4), which indicated the neural population started to encode the motor goals. The Euclidean distance was significantly larger than chance level (bootstrap test, $\mathrm{p}<$ 0.001; see Materials and Methods) and remained stable in the memory period. In PRR, the Euclidean distance between baseline states under normal and prism viewing context was significantly higher than chance level (Fig. 4A; bootstrap test, 
$\mathrm{p}<0.001$; see Materials and Methods). In PMd, there was no consistent result from the Euclidean distance analyses across two monkeys (Fig. 4B). The distributions of single-neuron activity were quite similar (Supplementary Fig. 1) across different viewing contexts; thus the observed separation before visual cue onset only emerged at the population level. In PRR, the Euclidean distance during baseline epoch was relatively stable across time. Adopting the language of dynamical systems, this activity during the baseline period seemingly converged toward an "attractor" state, a point in the neural state space to which the neural activity settles.

For comparison as a control, we repeated the Euclidean distance analysis on neural responses in block-designed pro-/anti-reach tasks, calculating the Euclidean distance between baseline activities. This analysis, by contrast, revealed similar baseline activity for pro- and anti-reach trials, even though they were alternated in blocks (Supplementary Fig. 2). This result, therefore, provided reassurance that the systematically shifted baseline state in prism viewing context indicated a real difference in neural state space. Depending on this initial state, visual stimulus triggers neural activity to follow distinct trajectories (corresponding to different directions) in either normal- or prism-trials. There was no difference with respect to the evolving speed of the activity pattern.

\section{The Link between baseline activity and the following motor goal encoding}

The initial state of brain activity interacts with stimulus input to shape the evolving neural trajectory. Most studies focus on movement control addressed the hypothesis that preparatory activity constitutes an initial state that largely determines the population-level pattern of movement activity (Churchland et al., 2010; Kaufman et al., 2014; Elsayed et al., 2016; Even-Chen et al., 2019). Dynamical systems perspective indicates that current neural population response 
should evolve predictably in neural state space. Thus, we asked how the population's baseline state in PRR consequently influences the subsequent neural activity associated with motor goal planning.

We developed a method that identified the optimal dimensions of the data on which motor goals representations were maximally separated in both normal and prism viewing contexts (encoding dimension). The direction-related activities on encoding dimensions became separate and easily interpretable. We then identified another dimension on which the baseline activity in normal- and prism-viewing contexts were maximally separated (baseline dimension). Neural activities were then projected onto these two dimensions separately, and followed by calculating the Euclidean distance between left- and right-reach trials. On the encoding dimension, the representation of motor goals was present as expected. The baseline dimension captured no encoding-related separation (Fig. 5), the distance between left- and right-reach trials were not significantly larger than chance level (bootstrap test, $\mathrm{p}<0.001$; see Materials and Methods). The results indicated that, during baseline, neural population activity in PRR avoids causing interference in motor goal representation by avoiding the key dimensions that are directly associated with motor goal planning.

\section{Discussion}

We investigated three potential kinds of computational strategies that could occur during the context-dependent sensorimotor transformation. The three strategies are corresponding to the factors which are used to describe a universal dynamical system: (1) the system's intrinsic dynamics, (2) the external inputs, and (3) the system’s initial state. Our result indicated that different computational strategies 
were employed independently, or in combination with others in different contextdependent sensorimotor transformation tasks. Reconfigurations of system's intrinsic dynamics and systematic adjustments of initial conditions were observed under the prism-reach task.

In both prism- and anti-reach task, spatial representation of a visual cue had to be remapped onto a spatially opposite motor goal during the visuomotor transformation, and the motor outputs of these two tasks were physically equivalent given identical visual cue. Yet, reach-related cortical areas solve these two context-specific tasks by employing different solutions. The different solutions might be associated with the learning strategy during task training. Both contextspecific tasks were trained after the monkeys became experienced in the "default" goal-directed reach task. On the one hand, monkeys could have treated the contextspecific task as an entirely different task. On the other hand, monkeys could have established a single solution that embeds the newly learned context-specific task into an existing continuum. Although we do not know the principles that led the brain to exploit different computational strategies, we speculate about potential computational advantages associated with each solution. For reach movement under prism viewing context, the contextual information was provided by a persistent cue with significant visual feature (prism). The reversed viewing context not only affected the visuomotor transformation but also perturbed the upcoming visual feedback once reach movement was initiated. The brain would function more efficiently by keeping the cue as a reliable signal across the whole trial (change system's intrinsic dynamics), instead of recruiting (receive external inputs) whenever the signal is needed. For anti-reach tasks, contextual information was fed into the system by temporary visual stimulus, the computations after visuomotor transformation (such as movement execution) were not affected by this 
cue. Thus, the neural population could simply activate neurons with different selectivity based on external inputs. It is not surprising that changing a neural manifold (covariance pattern) is more difficult than learning to use an existing neural manifold in novel ways. Our results further concluded that the universal computational strategy for space-context integration to encode the desired movement goal is not present. Neural populations change the computation they perform based on different behavioral contexts.

In addition to the computational strategies employed by different context-specific visuomotor transformations, our results indicated that population activity under the reversed viewing context systematically adjusted the initial conditions. The perturbation-evoked responses were initially restricted to motor goal independent dimension. This similar mechanism has been proposed to explain how movement preparation avoids premature movements during the instructed delay period of a reaching task (Kaufman et al., 2014). The present report extends this hypothesis to the case where viewing-related baseline activity needs to be prevented from affecting the upcoming visuomotor transformation. The neural manifolds seem to be widely present across brain and make a critical framework for describing previously unexplained neural computation.

\section{Figure captions:}

Figure 1. Tasks and Hypotheses. (A) Reversing-prism task. Monkeys viewed the screen monocularly through an aperture filled with a dove prism or left empty (upper left panel). They received visual feedback about their fingertips (vision of arm blocked by an opaque board) which was either congruent with their physical location (upper middle panel, normal viewing context) or reversed (upper right 
panel, prism viewing context). We thereby dissociated the physical and visual spatial parameters of the impending movement (physical goal and visual goal, respectively). Note that the visible portion of the fingertips is exaggerated here for illustration purposes. Timeline of the memory-guided center-out reach task in a single trial. In either viewing context, monkeys reached toward a peripheral visual stimulus (either left or right) after a random delay. (B) Pro/Anti-reach task. Timeline of the memory-guided center-out reach task in a single trial with context (colored square, instructing pro- or anti-reach) and spatial (black disc at 0, 90, 180, or 270 position). (C-D) Schematic of two hypotheses in an example threedimensional state space. Activity of three hypothetical neurons is involved in two computations. The activity of the three neurons in each kind of computation occupies a subspace (green or red plane) of the full space of possible states.

Figure 2. Neural manifold alignment in PRR. (A) Variance alignment analysis indicates the percentage of prism-reach variance explained by the top ten principal components calculated from the default reach planning in normal viewing context. Red trace, data from prism-reach; black dashed lines indicate alignment values expected under complete alignment. CI, confidence interval for the distribution drawing from random sampling. (B) Same as (A) except the vertical axis shows the percentage of anti-reach variance explained by the top ten principal components calculated from pro-reach data. Blue trace, data from anti-reach.

Figure 3. Neural manifold alignment in PMd. (A) Variance alignment analysis indicates the percentage of prism-reach variance explained by the top ten principal components calculated from the default reach planning in normal viewing context. 
Red trace, data from prism-reach; black dashed lines indicate alignment values expected under complete alignment. CI, confidence interval for the distribution drawing from random sampling. (B) Same as (A) except the vertical axis shows the percentage of anti-reach variance explained by the top ten principal components calculated from pro-reach data. Blue trace, data from anti-reach.

Figure 4. Pair-wise Euclidean distance in normal- and prism-reaches. (A) PRR neural activity. Euclidean distance in the full neural state space between each time point along the neural trajectories in the range of [-200 $500 \mathrm{~ms}]$ aligned to visual cue onset. The time point is in steps of $10 \mathrm{~ms}$. Green and red lines show the distance between left- and right-trials under normal (green) and prism (red) viewing context. Orange lines show the distance between trajectories corresponding to baseline activity under normal and prism viewing context, respectively. The thick part on each line indicates the time point at which the distance between two neural trajectories is significantly higher than chance level (bootstrapping procedure with 1000 resamples, $\mathrm{p}<0.001$; see Materials and Methods). (B) Same as (A) except for PMd neural activity.

Figure 5. Pair-wise Euclidean distance on single dimension. PRR neural activities were projected onto the first encoding dimension ( $k=1$, dark color) and baseline dimension (light color), separately (see Materials and Methods). On each dimension, Euclidean distance between two neural trajectories is aligned to visual cue onset. Green and red lines show the distance between left- and right-trials under normal (green) and prism (red) viewing context. The thick part on each line indicates the time point at which the distance between two neural trajectories is 
significantly higher than chance level (bootstrapping procedure with 1000 resamples, $\mathrm{p}<0.001$; see Materials and Methods).

Supplementary Figure 1. The histogram shows the distribution of single-neuron firing rates during baseline period (-200 to 0 ms aligned to visual cue), in normal and prism viewing context.

Supplementary Figure 2. Pair-wise Euclidean distance in block-designed pro/anti-reach. (A) PRR neural activity. Euclidean distance in the full neural state space between each time point along the neural trajectories in the range of [-200 0 $\mathrm{ms}$ ] aligned to visual cue onset. The time point is in steps of $10 \mathrm{~ms}$. Green and blue lines show the distance between left- and right-trials in pro- (green) and anti- (blue) conditions. Black lines show the distance between trajectories corresponding to baseline activity in pro- and anti-conditions, respectively. The thick part on each line (if present) indicates the time point at which the distance between two neural trajectories is significantly higher than chance level (bootstrapping procedure with 1000 resamples, $\mathrm{p}<0.001$; see Materials and Methods). (B) Same as (A) except for PMd neural activity. 
Figure 1

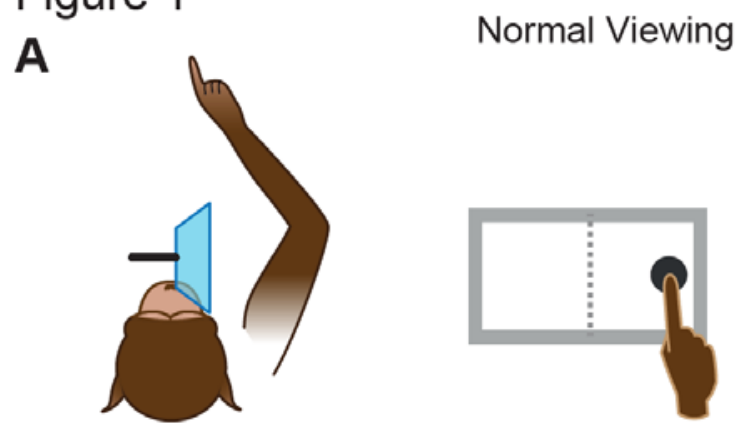

Prism reaches

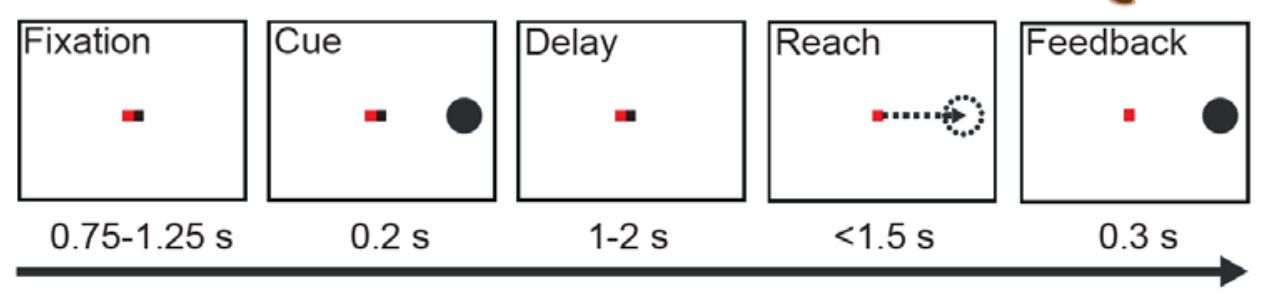

B

PRO reaches
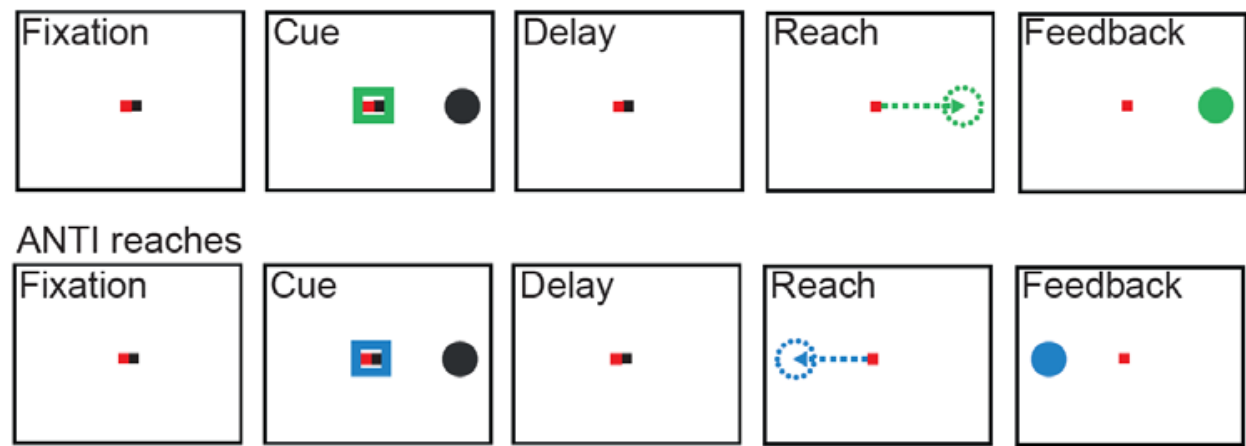

$0.5-1 \mathrm{~s}$
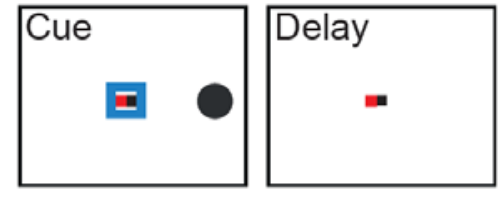

$0.8-2 \mathrm{~s}$

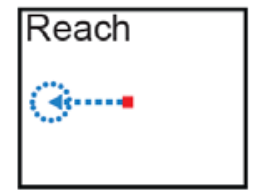

0.7-1 s

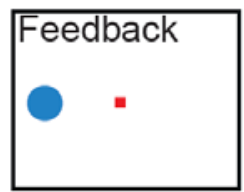

0.3-0.4 s

C

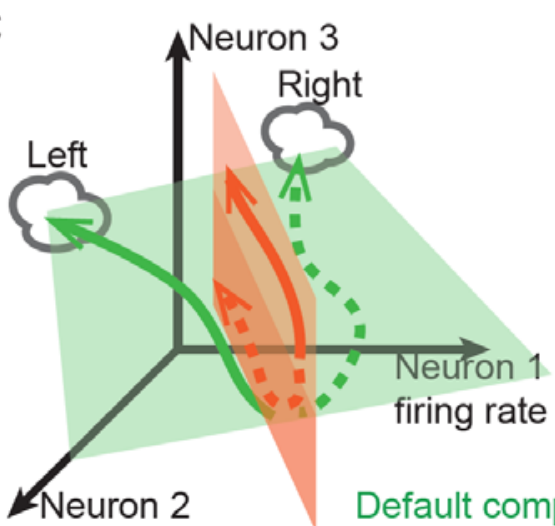

D

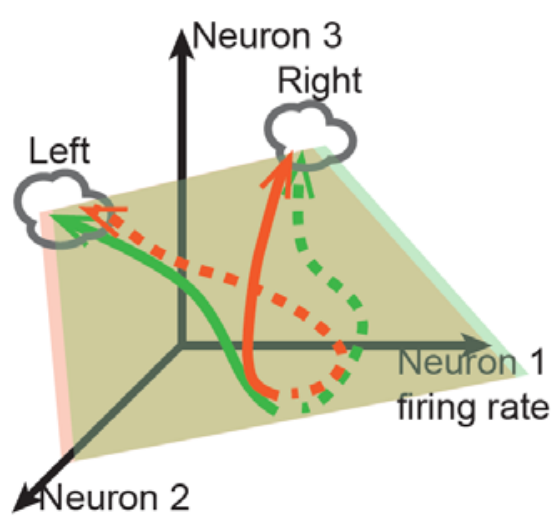

Context-specific computation 
Figure 2
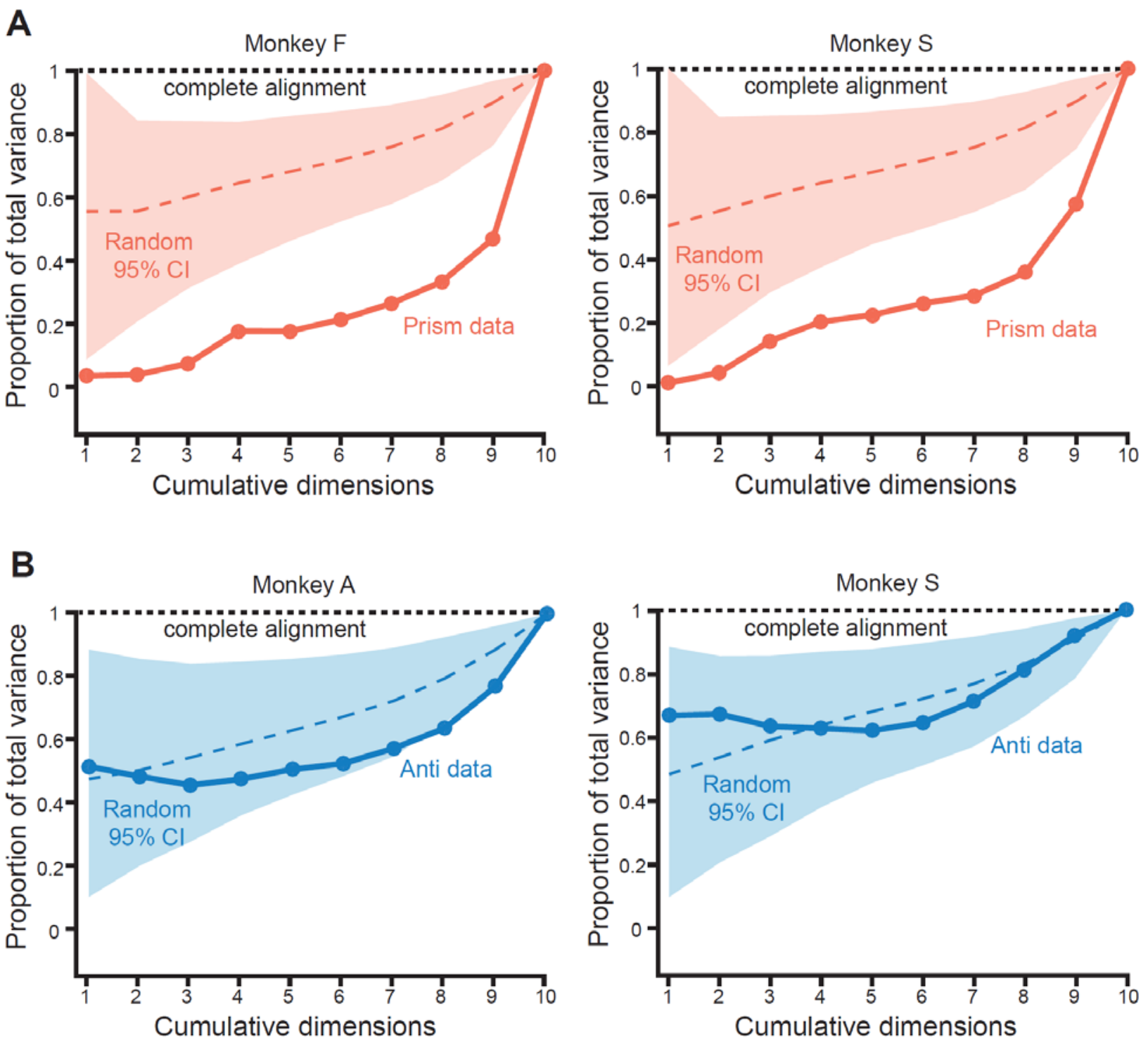
Figure 3
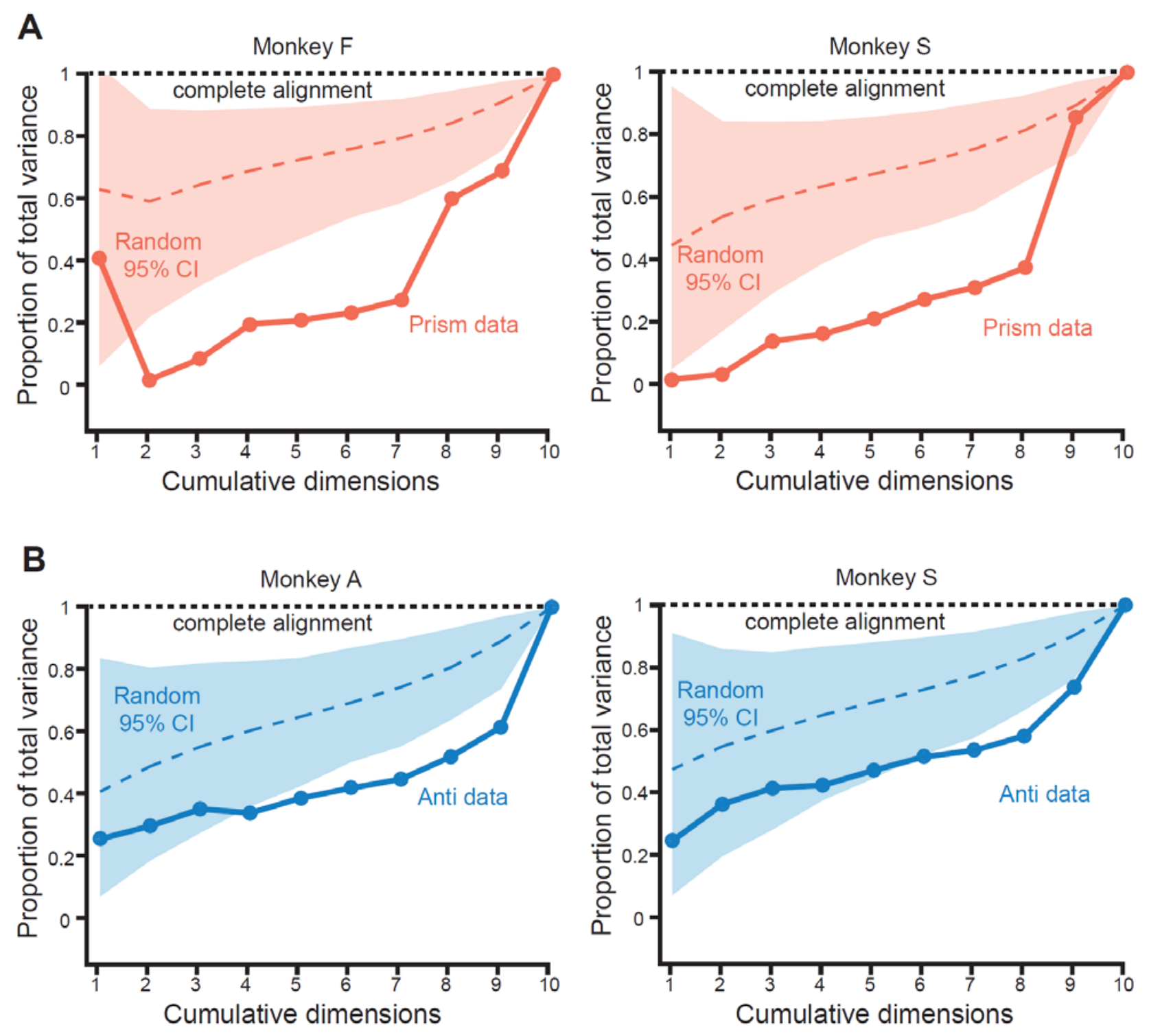
Figure 4
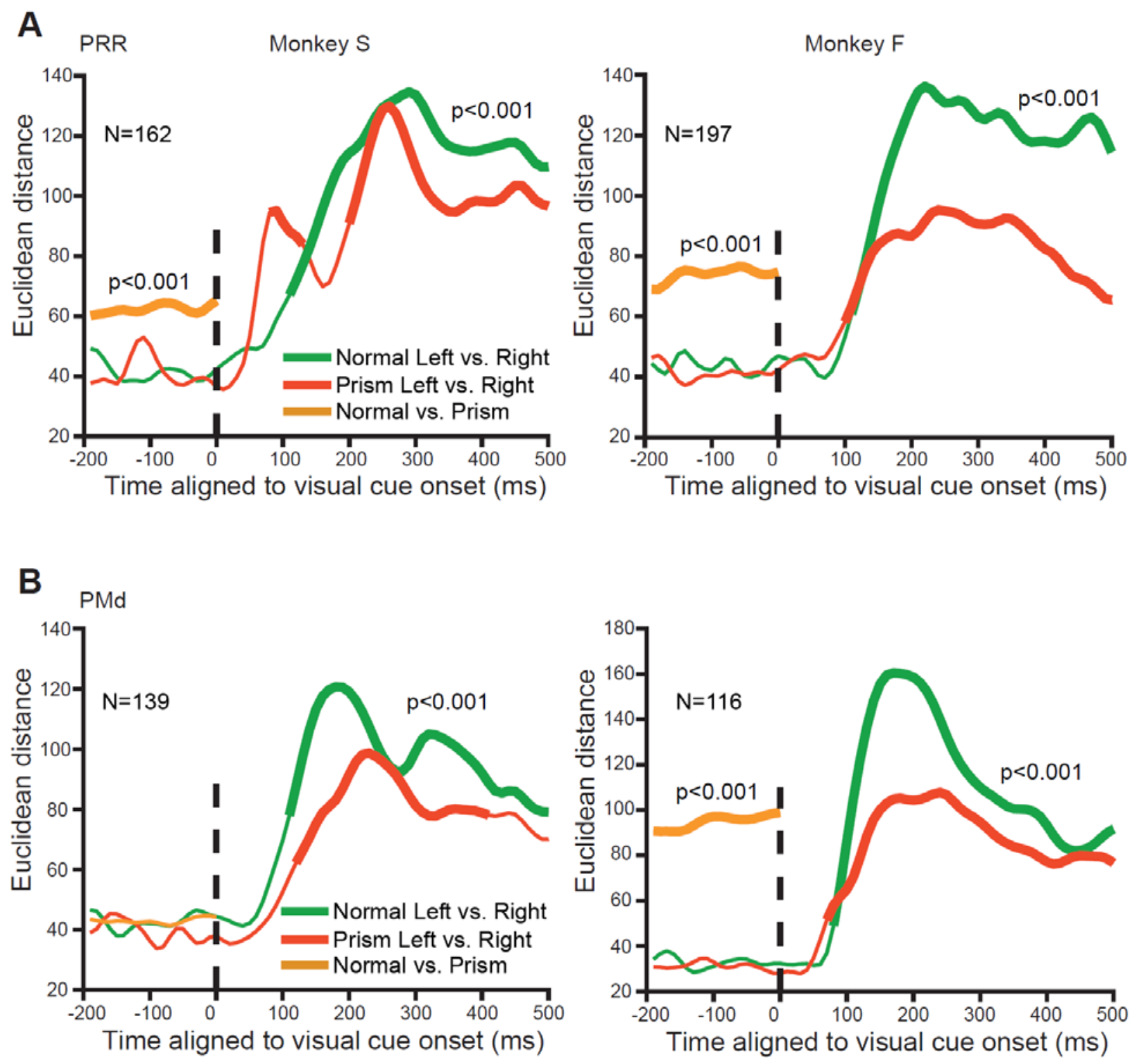
Figure 5
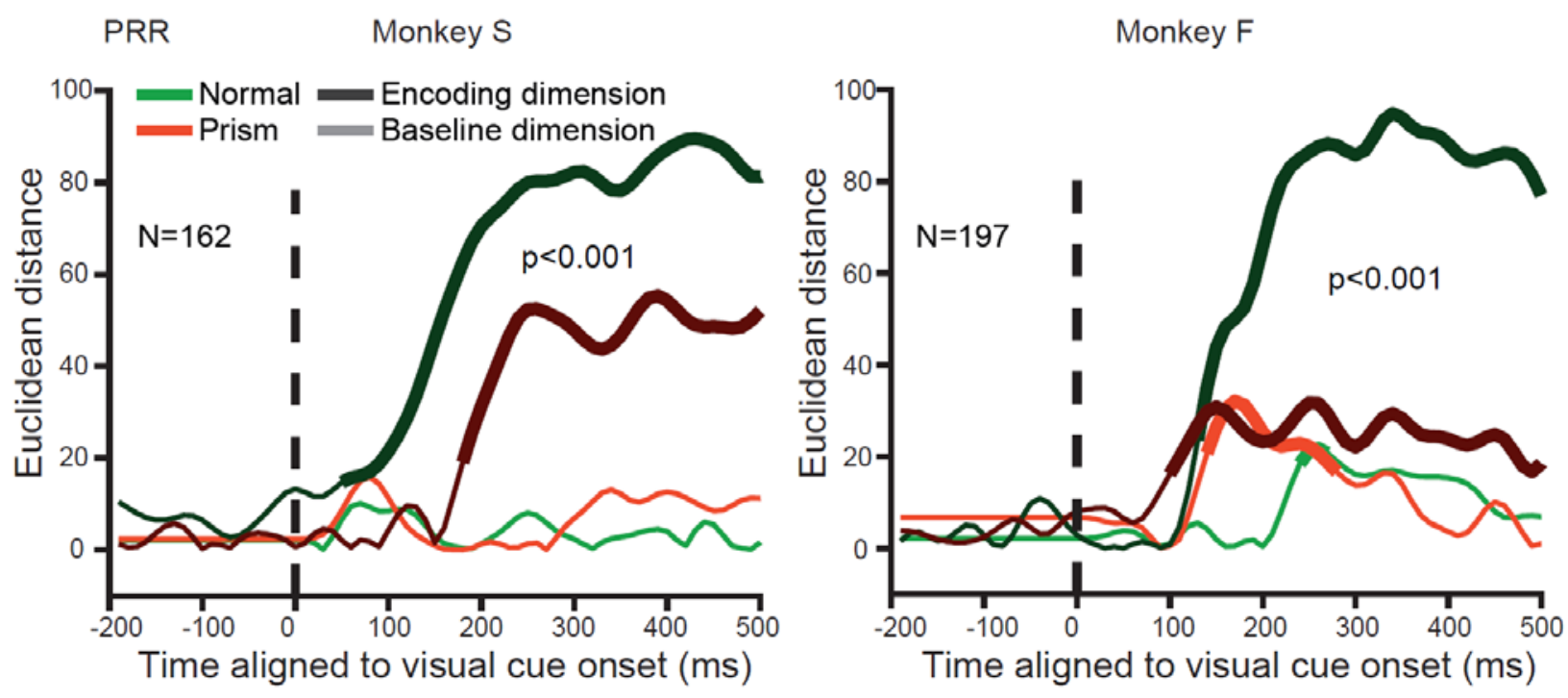


\section{Supplementary Figure 1}

A

PRR
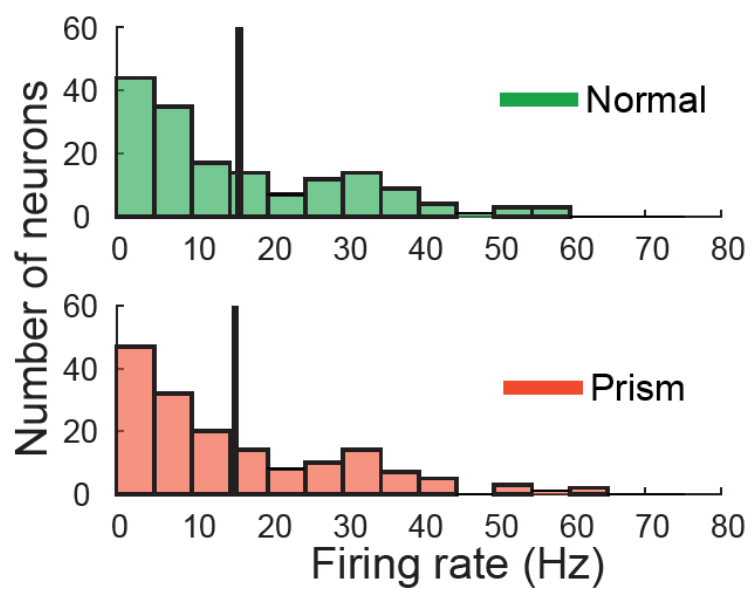

B

\section{PMd}

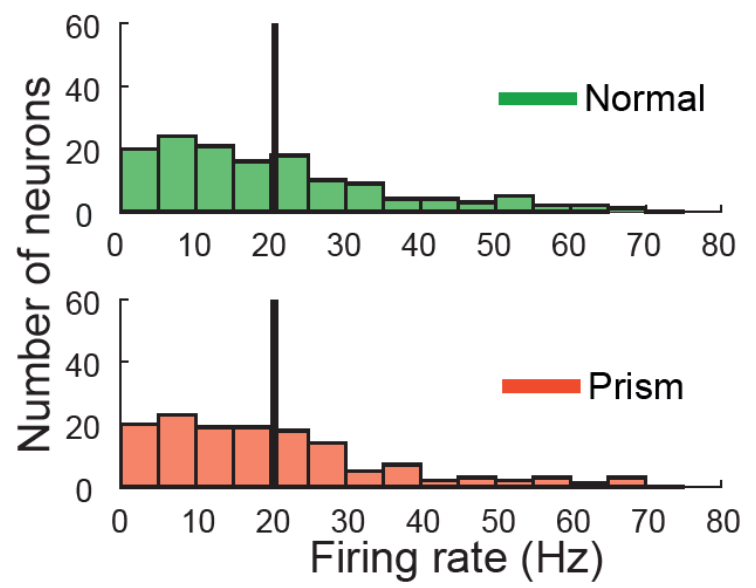

Monkey F
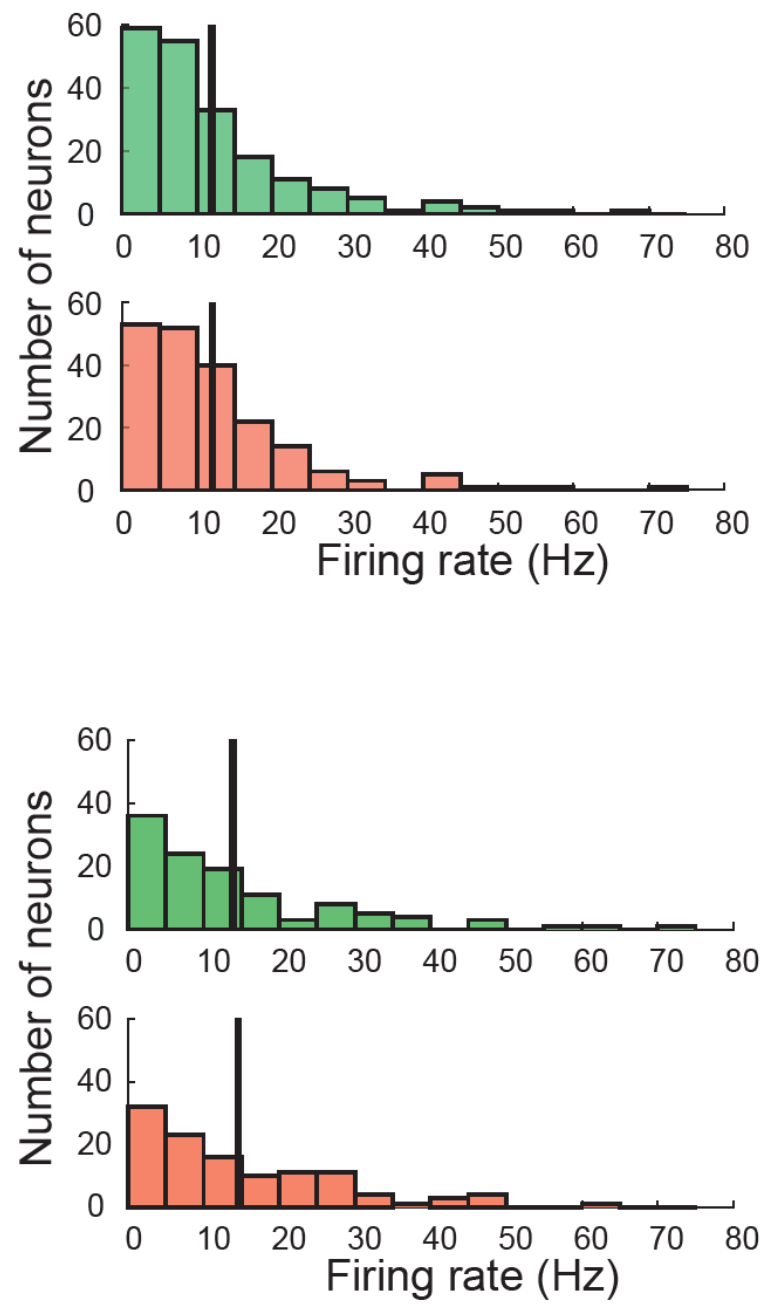


\section{Supplementary Figure 2}
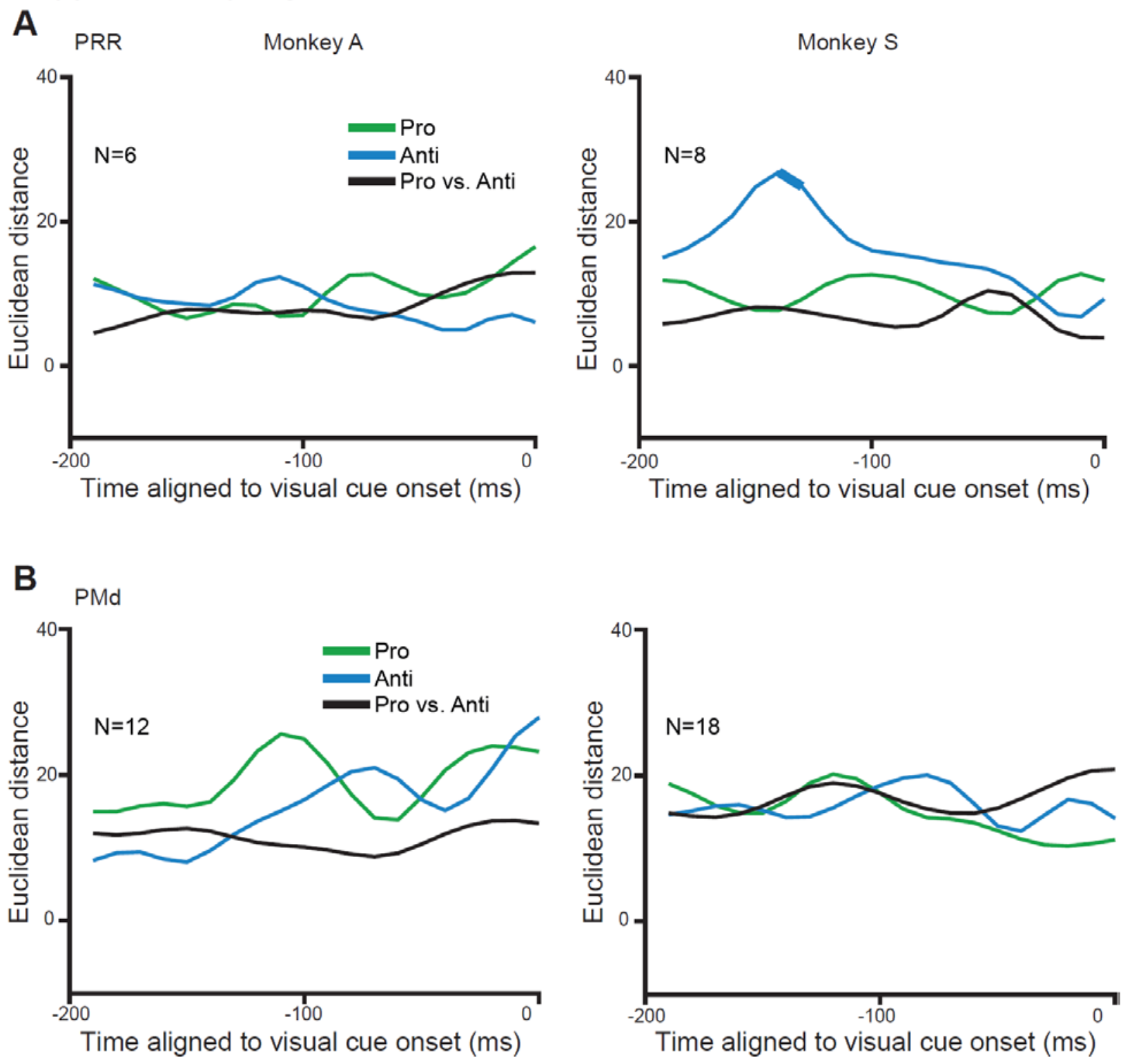


\section{References}

Churchland MM, Cunningham JP, Kaufman MT, Ryu SI, Shenoy KV (2010) Cortical preparatory activity: representation of movement or first cog in a dynamical machine? Neuron 68:387-400.

Cunningham JP, Yu BM (2014) Dimensionality reduction for large-scale neural recordings. Nat Neurosci 17:1500-1509.

Elsayed GF, Lara AH, Kaufman MT, Churchland MM, Cunningham JP (2016) Reorganization between preparatory and movement population responses in motor cortex. Nat Commun 7:13239.

Even-Chen N, Sheffer B, Vyas S, Ryu SI, Shenoy KV (2019) Structure and variability of delay activity in premotor cortex. PLoS Comput Biol 15:e1006808.

Gail A, Andersen RA (2006) Neural dynamics in monkey parietal reach region reflect context-specific sensorimotor transformations. J Neurosci 26:9376-9384.

Gail A, Klaes C, Westendorff S (2009) Implementation of spatial transformation rules for goal-directed reaching via gain modulation in monkey parietal and premotor cortex. J Neurosci 29:9490-9499.

Gallego JA, Perich MG, Miller LE, Solla SA (2017) Neural Manifolds for the Control of Movement. Neuron 94:978-984.

Harvey CD, Coen P, Tank DW (2012) Choice-specific sequences in parietal cortex during a virtual-navigation decision task. Nature 484:62-68.

Hennequin G, Vogels TP, Gerstner W (2014) Optimal control of transient dynamics in balanced networks supports generation of complex movements. Neuron 82:1394-1406.

Kaufman MT, Churchland MM, Ryu SI, Shenoy KV (2014) Cortical activity in the null space: permitting preparation without movement. Nat Neurosci 17:440-448.

Klaes C, Westendorff S, Chakrabarti S, Gail A (2011) Choosing goals, not rules: deciding among rule-based action plans. Neuron 70:536-548.

Kuang S, Morel P, Gail A (2016) Planning Movements in Visual and Physical Space in Monkey Posterior Parietal Cortex. Cerebral cortex (New York, NY : 1991) 26:731-747.

Machens CK, Romo R, Brody CD (2010) Functional, but not anatomical, separation of "what" and "when" in prefrontal cortex. J Neurosci 30:350-360.

Mante V, Sussillo D, Shenoy KV, Newsome WT (2013) Context-dependent computation by recurrent dynamics in prefrontal cortex. Nature 503:78-84. 
Pesaran B, Nelson MJ, Andersen RA (2008) Free choice activates a decision circuit between frontal and parietal cortex. Nature 453:406-409.

Raposo D, Kaufman MT, Churchland AK (2014) A category-free neural population supports evolving demands during decision-making. Nat Neurosci 17:1784-1792.

Sadtler PT, Quick KM, Golub MD, Chase SM, Ryu SI, Tyler-Kabara EC, Yu BM, Batista AP (2014) Neural constraints on learning. Nature 512:423-426.

Shenoy KV, Sahani M, Churchland MM (2013) Cortical control of arm movements: a dynamical systems perspective. Annu Rev Neurosci 36:337-359.

Snyder LH, Batista AP, Andersen RA (1997) Coding of intention in the posterior parietal cortex. Nature 386:167-170.

Wallis JD, Miller EK (2003) From rule to response: neuronal processes in the premotor and prefrontal cortex. Journal of neurophysiology 90:1790-1806.

Wang J, Narain D, Hosseini EA, Jazayeri M (2018) Flexible timing by temporal scaling of cortical responses. Nat Neurosci 21:102-110.

Westendorff S, Klaes C, Gail A (2010) The cortical timeline for deciding on reach motor goals. J Neurosci 30:5426-5436.

Wise SP, Boussaoud D, Johnson PB, Caminiti R (1997) Premotor and parietal cortex: corticocortical connectivity and combinatorial computations. Annu Rev Neurosci 20:25-42. 


\section{3 - Optogenetic inhibition of premotor-to-parietal projections in rhesus monkeys reveals a causal role in rule-based sensorimotor transformations}

Preparing a rule-based goal-directed reaching requires the integration of sensory information and abstract contexts. This process is mediated by the frontoparietal network. Although previous studies addressed a hypothesis that the space-context integration might be achieved in frontal areas and the contextual information might be passed on to the parietal cortex, the direct evidence is still missing.

By optogenetically silencing PMd-to-PRR projections, we directly tested whether the dynamics of rule-based visuomotor transformations in PRR causally dependent on functional input from PMd. We found that the inhibition of PMd projections to the PRR resulted in heterogeneous neural modulations related to motor-goal representation in PRR. The directional selectivity could be preserved, erased or evoked by the pathway-selective optogenetic inhibition. Furthermore, as predicted by the hypothesis, inhibiting PMd-to-PRR projections increased the latency of motor-related tuning in PRR, exclusively during the rule-based sensorimotor transformations. 


\title{
Optogenetic inhibition of premotor-to-parietal projections in rhesus monkeys reveals a causal role in rule-based sensorimotor transformations
}

\begin{abstract}
Context-dependent sensorimotor transformations have been associated with the frontoparietal network in the cerebral cortex of primates. Although it is hypothesized that several areas in frontal and parietal cortex, which are anatomically connected, coordinate their activity for context-dependent motor goal selection, the causal link between interconnected areas remains to be elaborated. Here, using pathway-selective optogenetic methods, we reversibly inhibited the neural projections from the dorsal premotor area (PMd) in frontal cortex to the parietal reach region (PRR) in the parietal cortex of a macaque monkey performing a rule-based reach task (pro/anti-reach). We found that the optogenetic inhibition of local PMd projections to PRR at the level of single neuron activity results in heterogeneous neural modulations related to motor-goal representation in PRR. The directional selectivity of individual neurons could be preserved, erased or evoked by the pathway-selective optogenetic inhibition. We also investigated the temporal properties of motor goal tuning in PRR at the population level. The optogenetic modification increased the latency of motor-related tuning exclusively during the context-dependent sensorimotor transformations (i.e., when the task requires spatial remapping). These results support the hypothesis that dynamic reorganization in PRR during spatial remapping is contingent on the inputs from PMd.
\end{abstract}




\section{Introduction}

Behaviors such as sensing an object and then moving the hand toward it require that sensory information is converted into motor commands, a process known as sensorimotor transformation. In real life situation, behavior is not exclusively afforded by the sensory input. Context-dependent sensorimotor transformation refers to capabilities that allow a subject to perform different behavioral responses depending on contextual information, even though the sensory stimuli are identical. The dorsal premotor cortex (PMd) in the frontal lobe and parietal reach region (PRR; here: MIP) in the posterior parietal cortex of monkeys are believed to mediate such context-dependent sensorimotor transformation (Wallis and Miller, 2003; Gail and Andersen, 2006; Cisek, 2007; Pesaran et al., 2008; Gail et al., 2009). When the reach goal needs to be inferred from a spatial cue by applying a transformation rule, PMd and PRR encode spatial motor-goal information, not spatial cue-related information, during motor planning (Crammond and Kalaska, 1994; Gail and Andersen, 2006; Gail et al., 2009; Westendorff et al., 2010).

PMd and PRR share basic functional properties and are believed to work together through their reciprocally connected pathways in a collective manner. Numerous studies showed that PMd receives input from MIP (Johnson et al., 1996; Petrides and Pandya, 1999; Luppino et al., 2001; Marconi et al., 2001; Tanne-Gariepy et al., 2002; Luppino et al., 2003; Markov et al., 2014), as well as from V6A (Matelli et al., 1998; Caminiti et al., 1999; Gamberini et al., 2009). Injections in V6A (neighboring MIP and, according to some authors likely partially overlapping PRR) showed dense projections to PMd (Caminiti et al., 1999; Marconi et al., 2001; Gamberini et al., 2009; Bakola et al., 2010). Additionally, VA6 and MIP are mutually interconnected (Gamberini et al., 2009; Passarelli et al., 2011), which together argues for bidirectional connectivity between PRR (MIP/V6A) and PMd. 
The anatomical architecture of the frontoparietal reach network imposes constraints on the nature of their coordinate activity. How do reciprocally connected premotor and parietal areas functionally interact during the integration of sensory and contextual information? Latency analysis of neural spiking showed that PMd leads PRR in terms of representing context-dependent motor goals in the anti-reach task (Westendorff et al., 2010). This observation suggested that spacecontext integration might be achieved in frontal areas and the resulting motor-goal information might be passed on to the parietal cortex. The frontal area leading parietal area was also observed when internally represented context had to be integrated with the spatial information in decision-related reach task (Pesaran et al., 2008). Spike-field coherence suggested that the PMd to PRR link is activated first, followed by a hand-shake back from PRR to PMd. Data from modeling (Brozovic et al., 2007) and physiological experiments (Pesaran et al., 2008; Westendorff et al., 2010) converge in suggesting that the dynamic reorganization of network activity in PRR is contingent on frontal-parietal projections from PMd. The premotor-toparietal projections functioned exclusively when contextual information is involved in sensorimotor transformation.

While studies of single-cell activity and modeling support this hypothesis, a recent study based on Granger-causality measure of intracortical local field potentials argued against the functional interaction within the frontoparietal network (Martinez-Vazquez and Gail, 2018). This study showed that low-frequency PMd activity had a transient Granger-causing effect on PRR specifically during working memory retrieval of spatial motor goals, while no frontoparietal directed interaction was associated with visuomotor transformations. Thus, the functional role of the inter-areal interaction between premotor and posterior parietal cortex is not clear from the inconsistent results reported previously. 
Are the dynamics of rule-based visuomotor transformations in PRR causally dependent on functional input from PMd? In order to provide causal evidence to answer this question, optogenetic silencing of synaptic terminals (of PMd) by local illumination of the target region (PRR) would be the method of choice. We used pathway-selective optogenetic suppression to inhibit the presynaptic terminals of

PMd projecting to PRR. Optogenetic tools (Deisseroth, 2015; Grosenick et al., 2015) have been used to expand our understanding of the brain's functions (Galvan et al., 2017). Neurons can be genetically modified to express eArchT3.0, a greenlight-sensitive opsin (532 nm wavelength) that pumps protons out of cells (Han et al., 2011). The optical stimulation of eArchT3.0 expressed on axon terminals in the downstream brain areas inactive the synaptic response and can, therefore, inhibit signal transmission between two brain areas. Such pathway-selective optogenetics has advanced our understanding of the roles of particular neural pathways in a variety of behaviors (Stuber et al., 2011; Tye et al., 2011; Warden et al., 2012; Inoue et al., 2015; Galvan et al., 2016). In this study, we speculated that if the information flows from PMd to PRR carry context-related information, by partially blocking the PMd-to-PRR projections, PRR would exhibit delayed motor-goal tuning exclusively when spatial remapping is required, because the space-context integration is disturbed in that case.

\section{Materials and Methods}

All experimental procedures were conducted in accordance with institutional guidelines on Animal Care and Use of the German Primate Center, the European Directive 2010/63/EU, the corresponding German national law and regulations 
governing animal welfare, and were approved by regional authorities where necessary.

\section{Behavioral task}

One adult male rhesus monkey (Monkey A) was trained to perform a memoryguided center-out anti-reach task. The monkey was required to manipulate a joystick to guide a cursor on a computer screen mounted in the frontoparallel $2 \mathrm{D}$ plane. The anti-reach task required the monkey to map a spatial cue onto one of two motor goals, either at the location of the visual cue (pro-reach) or opposite to it (anti-reach). The transformation rule was instructed with a colored frame around the central eye fixation spot (Fig. 1A) (see below for details). The four peripheral and color neutral spatial cue (right, $0^{\circ}$; up, $90^{\circ}$; left, $180^{\circ}$; down, $270^{\circ}$ direction) was centered around the central fixation point at $8 \mathrm{~cm}$ eccentricity.

The timeline of the trials was as follows (Fig. 1A): The monkey initiated a trial by acquiring central eye fixation at a small red spot (registered with an infrared camera, EyeLink 1000 Plus, SR Research Ltd.) and moving the cursor within a hand fixation area which was defined by a grey disc surrounding the red eye fixation spot. A variable-length fixation period (800-1200 ms) was followed by a brief visual cue period (200 ms). The peripheral spatial cue and contextual cue were flashed simultaneously. The contextual cue presented in this period consisted of a colored frame around the small, central red eye fixation spot, and instructed the monkey to reach toward (pro-reach; green cue) or diametrically opposite (antireach; blue cue) of the previously flashed spatial cue. For a variable duration (800$1200 \mathrm{~ms}$ ), the monkey had to keep both eye (tolerance of $3.5^{\circ}$ of visual angle) and hand fixation (memory period). When the central hand fixation target turned off (go signal), the monkey had to reach toward the instructed goal. The monkey 
received visual feedback about the correct motor goal (circular stimulus at the desired location) if he moved the cursor into the correct target, or after the maximum movement period (500 ms) expired, in which case the trial was aborted. The monkey had to keep the cursor at the reach target location (feedback period, $200 \mathrm{~ms}$ ) to successfully finish the trial and receive a liquid reward.

\section{Surgery and injection of viral vector}

Two custom-fit recording chambers were implanted on the monkey's skull contralateral to the handedness of the monkeys. The implantation of each chamber, one for PRR and the other for PMd, was guided by pre-surgical MRI and confirmed by post-surgical MRI. The precise coordinates for virus injection in PMd were calculated based on the MRI after the chamber implantation. The MR imaging procedures, chamber implantation surgery, and virus injection were all conducted under general anesthesia.

Viral vector injections in PMd were performed after the chambers were implanted. To cover a large region of area PMd, conventional viral injection techniques for monkeys require multiple small-volume injections. In PMd area, we located four injection sites that were spaced 1.5-2.7 mm on either Anterior-Posterior (AP) or Medial-Lateral (ML) direction (Fig. 1B and C). On each site, three depths spaced 0.7-1.0 mm apart were used for injection, the different depths were along a track which is perpendicular to the brain surface. A small incision on dura was made for each injection site to facilitate the penetration. A microinjection Hamilton syringe (\#701) loaded with $9 \mu \mathrm{l}$ of virus (AAV2/5-CaMKII $\alpha$-eArchT3.0-eYFP; titer = $4 \times 10^{12} \mathrm{vg} / \mathrm{ml}$; UNC Vector Core) was advanced into area PMd using an electric microdrive. We first advanced the tip of the needle to the deepest point $(2.5 \mathrm{~mm}$ to the putative lower surface of the dura) of each injection site and began the first of a 
series of injections. Using the syringe Microdrive, we injected 1-1.5 $\mu \mathrm{l}$ at a rate of $250 \mathrm{nl} / \mathrm{min}$. Each injection was then followed by a 5 min wait period before slowly retracting the needle to the next depth. This process continued until reaching the shallowest point of each injection site, resulting in 12 injections and a total of $16 \mu \mathrm{l}$ injected into PMd. To examine viral vector-mediated opsin expression histologically, we injected two additional monkeys (monkey $\mathrm{O}$ and $\mathrm{G}$ ) following a similar procedure as in monkeys A.

\section{Neural data acquisition}

After subjects became proficient in the anti-reach task, neural activity of PRR and PMd were recorded with 1-4 electrodes (Thomas Recording, Giessen, Germany) in each area in each session (Fig. 1C, D). The $\mathrm{x}$-y electrode locations within the chamber were positioned in each recording session using the XYZ-manipulator that holds the Microdrive (Thomas Recording, Giessen, Germany) with submillimeter resolution. The recording coordinates in each chamber were estimated from post-surgical MRI. At the halfway between pre-central dimple, arcuate spur and superior arcuate sulcus for PMd (Fig. 1C), the recording sites were at approximately 1-2.5 mm below the cortical surface. Along the medial wall of IPS for PRR (most likely MIP; Fig. 1D), we performed recordings at a depth of approximately 3-7 $\mathrm{mm}$ from the cortical surface.

We used a five-channel microdrive ("mini-matrix"; Thomas Recording) for extracellular recordings in combination with optical stimulation. One channel of the mini-matrix was loaded with an optical fiber (Thomas Recording, Giessen, Germany), while the remaining four were loaded with electrodes. The horizontal distance between the optical fiber and each electrode was $500 \mu \mathrm{m}$. After advancing the electrodes and optical fiber into area PMd and pausing 20-30 min to allow the 
tissue to stabilize, we isolated single-unit activity using Plexon SortClient software (Plexon, Inc.). During extracellular recording, the inter-tip linear distance between each electrode and optical fiber was $500-950 \mu \mathrm{m}$. The raw signals of the electrodes were preamplified (20×; Thomas Recording, Giessen, Germany), band-pass filtered into broadband data (154 Hz to $8.8 \mathrm{kHz})$ and LFPs $(0.7$ to $300 \mathrm{~Hz})$. The band-pass filtered LFPs were digitized and sampled at $1000 \mathrm{~Hz}$ (Plexon MAP system, Dallas, TX, USA). Spike times, spike waveforms and broadband signals were recorded and later subjected to additional offline sorting (Offline Sorter; Plexon). All well-isolated neurons were recorded, and no attempt was made to screen for neuronal tuning for reach direction or any other response property.

\section{Optogenetic inhibition protocol}

Prior to each recording session, we used a handheld power meter (PM100D, Thorlabs $\mathrm{GmbH}$ ) to calibrate the output of 532-nm green laser power as a function of analog input (from teensy microcontroller) to the laser controller (LRS-0532 DPSS Laser System, Laserglow Technologies). The light power was kept between 12 and $16 \mathrm{~mW}$ at the tip of the optical fiber. The neural activity was recorded by randomly interleaving optical stimulation trials (Opto-Stim) with no-laser trials (Non-Stim) with 50\% chance. In Opto-Stim trials, including pro-reach and antireach trials, a continuous 330ms laser pulse began simultaneously with the display of the spatial cue and context cue on the screen (Fig. 1E). The laser power profile was a constant square pulse and was terminated immediately when the analog input to the laser controller was shut down. In 30\% recording sessions in PRR, laser onset began about 30-50 ms before the visual cue onset to ensure the optogenetic inhibition clamped the axon terminals before the earliest PMd inputs reached PRR. In combination with eight reach conditions in the behavioral task, which were also randomly interleaved trial by trial, 16 conditions in total [two 
context conditions $\times$ four directions $\times$ two optical conditions (Opto-Stim and NonStim)] were pseudo-randomly interleaved, and the presence of laser illumination was not indicated to the animal.

\section{Directional selectivity in individual neurons}

The directional selectivity analysis was conducted in Non-Stim and Opto-Stim condition separately, to reveal the effects of optical stimulation on single-neuron activity. We defined the cue epoch as a 250 ms interval beginning $100 \mathrm{~ms}$ after visual cue onset. This epoch started after the stimulus-evoked activity had developed and covered the whole period when laser stimulation was on. We defined the early memory epoch as a $250 \mathrm{~ms}$ interval beginning $150 \mathrm{~ms}$ after the visual cue offset, which avoided any overlap with the laser stimulation if present. Directional selectivity was quantified in each epoch with a directional tuning vector (DTV). It was defined as the vector average across the four directions $\vec{u}_{d}$ (unit vectors) weighted with the corresponding mean spike rates $r_{d i}$ of neuron $\mathrm{i}$ as follows:

$$
\mathrm{DTV}_{i}=\sum_{d=1}^{4} r_{d i} \vec{u}_{d}
$$

The direction of the DTV can be any value between $0^{\circ}$ and 360 . The DTV was computed in each epoch, separately for pro and anti-trials, and separately within each laser stimulation condition (opto- or non-stim). The significance of directional tuning was defined as spike rate significantly different among four directions and tested with a nonparametric one-way ANOVA (Kruskal-Wallis, $\mathrm{p}<0.05$ ). Based on the tuning properties in Non-Stim and Opto-Stim condition, the recorded neurons were categorized into four groups: (1) neurons exhibited significant directional selectivity in both Non-Stim and Opto-Stim condition ("preserved" 
group); (2) neurons which were selective in Non-Stim trials fail to possess selectivity in Opto-Stim trials (“erased” group); (3) neurons without selectivity in Non-Stim condition became selective in Opto-Stim condition (“evoked” group); (4) neurons without significant directional selectivity in either condition ("neither" group).

\section{Population analyses of motor-related tuning}

We calculated average peri-stimulus time histograms (PSTHs) across neurons within each task condition. We did this to describe the neural response properties as a function of time. PSTHs across neurons were calculated as the mean PSTHs in the direction of each neuron's maximal response (MD stands for maximum direction) and, separately, in the opposite direction (NP stands for non-preferred direction). The MD for each neuron was defined as the direction with the maximal spike rates during early memory epoch of Non-Stim trials (Opto-Stim trials if neuron belongs to "evoked” group), in either rule cue condition (pro or anti). It is noteworthy that the MD and NP directions were restricted to the discrete spatial cue direction. The PSTH of each neuron was first normalized to its maximal response over all 4 directions during the early memory epoch of Non-Stim trials (Opto-Stim trials if neuron belongs to “evoked” group), followed by averaging across neurons. This calculation was conducted separately for pro- and anti-trials.

\section{Quantitative analyses of optogenetic effects}

We estimated the time during which optogenetic inhibition significantly affects directional selectivity, either in pro- or anti-condition by using a permutation test. PSTHs between two optical conditions (Non-Stim and Opto-Stim) at MD direction were computed and tested for being different from zero. Permutation tests ( $\mathrm{N}=1000$ samples) were used to estimate the probability that the original sample 
indicates a significant difference from zero. The permutation tests were applied to pro- and anti-reach data separately. In each permutation, each neuron's PSTH at MD direction was randomly assigned to one of the two optical conditions. The resulting $\mathrm{p}$-value represents the percentage of random permutations leading to a PSTHs difference larger or equal to the original sample. For all the time points in the data, a difference was considered significant if the p-value was $<5 \%$.

Permutation test for averaged PSTHs across neurons was also used to estimate the latency of motor tuning within each condition at which the neural responses to the MD and NP became significantly different; that is, the time when motor-goal encoding emerges. In each permutation, each neuron's PSTH data were randomly assigned to one of the two directions (MD or NP), the p-value at each time point represents the percentage of permutations that are larger than the original sample. The latency in the Non-Stim and Opto-Stim condition, respectively, was defined as the time when the p-value dropped below 5\% and stayed below $5 \%$ at least in the successive 20 bins (200 ms). In order to decide if the latency difference between Non-Stim or Opto-Stim condition is statistically significant, we randomly assigned neuron's PSTH data to one of the two conditions (Non-Stim or Opto-Stim) in MD and NP direction separately, and calculate the latency time for each permutation sample following the procedure as mentioned before. The resulting p-value represents the percentage of random permutations leading to a latency difference larger or equal to the original sample. A difference was considered significant if the p-value was $<5 \%$. 


\section{Results}

To investigate the functional role of the premotor-to-parietal projections in context-dependent sensorimotor transformation, one macaque monkey (Monkey A) was trained to perform instructed-delay center-out pro-/anti- reaching tasks (Fig. 1A). To deliver the eArchT3.0 gene into targeted neurons, we injected AAV2/5CaMKII $\alpha$-eArchT3.0-eYFP into the PMd of the left hemisphere (Fig. 1B) in monkey A (for neurophysiology experiment). For the expression in rodent and rhesus macaque cortical neurons, a well-established proton pump, ArchT, has been proved to be expressed well on neuronal membranes, including trafficking for long distances down neuronal axons (Han et al., 2011). In this research, we chose eArchT3.0, which is the enhanced counterpart of ArchT. For long-range axonal projections such as PMd to PRR, several weeks are necessary to obtain sufficient opsin expression in axonal terminals. This animal continues to participate in experiments and is unavailable for histology. Therefore, to test the overall efficacy of the AAV2/5-CaMKIIa vector and identify the premotor-to-parietal projections, we performed immunohistochemical analysis in two additional animals (Monkey $\mathrm{O}$ and $\mathrm{G}$ ) following injecting AAV2/5-CaMKIIa-hChR2-eYFP into the PMd of the left hemisphere. After 8.5 weeks, we histologically confirmed the hChR2-eYFP expression in PMd. ChR2-positive neurons were found in the PMd (Fig. 2A). We also observed many ChR2-positive axon terminals in the PRR (MIP) area ipsilateral to the vector-injected hemisphere (Fig. 2C). The result confirmed the monosynaptic projections from PMd to PRR.

We applied optogenetic inhibition in either PMd (Fig. 1C) or PRR (Fig. 1D) area using 532-nm green laser and simultaneously recorded single-neuron activity. Extracellular single-neuron spiking activities were recorded from 137 neurons in PMd and 311 neurons in PRR while the monkey performed the anti-reach task (see 
Materials and Methods). On half of the trials, the green laser was on, while on the other half the laser was absent. In the optical stimulation trials, the continuous laser light emission lasted $330 \mathrm{~ms}$ beginning right with visual cue onset (Fig. 1E). In some recording sessions, the laser began $30-50 \mathrm{~ms}$ before visual cue onset to ensure the optogenetic inhibition clamped the axon terminals before the earliest PMd inputs reached PRR. These two optogenetic inhibition protocols did not lead to different results in all analyses; thus we group all recording sessions for analyses. During each recording session, all 16 task conditions [two context conditions $\times$ four directions $\times$ two optical conditions (Opto-Stim and Non-Stim)] were pseudorandomly interleaved. In the Non-Stim trials, the monkey performed the reaching task with high performance. The overall success rate was $71 \%$ in both pro- and anti-reaches. Errors were mainly caused by ocular fixation failures, not by incorrect target choices. The choice of reach target was correct in 99\%. In OptoStim trials, the local optogenetic inhibition in PMd and pathway-selective optogenetic suppression in PRR did not show any behavioral modulations.

\section{Neuronal modulations in PMd and PRR by optogenetic inhibition}

As a first step in understanding the premotor-to-parietal interactions, we began by assessing single-neuron firing properties. We first examined whether local optical stimulation of PMd affected single-neuron firing. We found that 66 of 137 recorded neurons exhibited a changed response during optogenetic stimulation and 61 of 137 neurons exhibited significant suppression (Wilcoxon signed-rank test, P $<0.05$ ) (see Fig. 2B for a complete suppressed PMd neuron example). The offset of the photo-stimulus then produced a burst of excitation (so-called rebound response) in most of the affected PMd neurons. 
We then examined whether optical stimulation of PMd axon terminals affects postsynaptic neuronal spiking in the PRR. Regardless of tuning properties, 63 of 311 neurons exhibited significant firing changes (Kruskal-Wallis, $\mathrm{p}<0.05$ ) at least in one direction in the pro-reach trials, 53 of 311 neurons in anti-reach trials, ten neurons in both pro- and anti-reach trials (see Fig. 2D for a suppressed PRR neuron example).

\section{Heterogeneous changes of PRR tuning profile under optogenetic inhibition}

The above data suggest that the optogenetic approach modulated PRR neural activity in a pathway-selective manner. We then examined whether the pathwayselective optogenetic inhibition was sufficient to change the tuning properties of single neurons in PRR. Based on the tuning properties in both Non-Stim and OptoStim conditions, the recorded neurons in PRR were categorized into four groups (see Materials and Methods). The categorizations were performed separately in pro- and anti-reach trials. 51 (pro) and 43 (anti) of 311 neurons exhibited significant directional selectivity in both Non-Stim and Opto-Stim conditions (“preserved” group). Figure 3 shows the population PSTHs (see Materials and Methods) of all "preserved" neurons in PRR for pro- and anti-reaches (Fig. 3A, B). The population PSTHs in Non-Stim and Opto-Stim condition were practically identical, indicates that these neurons were not affected by the optogenetic inhibition.

There were 42 (pro), and 36 (anti) of 311 neurons with significant selectivity in Non-Stim trials failed to possess significant directional tuning in Opto-Stim trials (“erased” group) (Fig. 4A, B). In Opto-Stim trials, the amplitude of the average neural response to the MD significantly decreased ( $\mathrm{p}<0.05$, permutation test), even after the offset of the laser stimulation. It is noteworthy that even though the 
directional selectivity of single neuron was "erased" in the sense of significance, the neural population response still encoded the motor goal (separation between the neural responses to the MD and NP, $\mathrm{p}<0.05$, permutation test) in pro-reach trials (Fig. 4A), whereas in anti-reaches the representation of motor goal was thoroughly erased by optogenetic inhibition (Fig. 4B).

Previous studies proposed the hypothesis that the dynamic reorganization of network activity in PRR is contingent on frontal-parietal projections from PMd. By inhibiting PMd-to-PRR projections, intuitively, we would assume that PRR neurons lost their ability to encode correct motor goals (as showed in the "erased" group). Interestingly, we observed a group of neurons without significant tuning in Non-Stim condition exhibited significant selectivity (“evoked” group) under OptoStim conditions (Fig. 4C, D). Directional selectivity in 47 (pro) and 39 (anti) neurons emerged from optogenetic inhibition, and the average neural response to the MD therefore significantly increased ( $p<0.05$, permutation test). The fact that PRR neurons could be categorized into four groups ("preserved," "erased,” “evoked," and "neither”) based on their tuning properties in Non-Stim and OptoStim condition, suggests the heterogeneous roles of PMd inputs on different PRR neurons.

\section{Effect of optogenetic inhibition on motor-goal latency}

The latency of motor tuning within each condition was defined as the time relative to visual cue onset at which the neural responses to the MD and NP were significantly different. We compared motor-goal latencies between Non-Stim and Opto-Stim within either pro-reach or anti-reach trials. Only neurons that showed significant directional selectivity in Non-Stim condition were included in this analysis. In both pro- and anti-trials, the population PSTHs in Opto-Stim condition 
significantly decreased during early memory period (Fig. 5A, B), indicating that optogenetic inhibition of the PMd projections to the PRR temporally attenuated the representation of motor goal in PRR. In the pro-trials, the latencies of motor-goal encoding in Non-Stim and Opto-Stim conditions were practically identical (Fig. 5A). In the anti-trials, the average PRR PSTHs for motor-goal encoding had a higher latency in Opto-Stim compared to Non-Stim (Fig. 5B) (Opto - Non $=70 \mathrm{~ms}$, $\mathrm{p}<0.05$, permutation test).

\section{Discussion}

Our viral transfection protocol allowed us to locally light-inactivate PMd neurons and remotely, in PRR, we putatively light-inactivated specific projections from PMd to PRR. We used this transient optogenetic inhibition to selectively inhibit excitatory neurons in PMd and the PMd-to-PRR projections during visuomotor transformation in a rule-based goal-directed reach task. We found that the optogenetic inhibition of PMd projections to the PRR results in heterogeneous neural modulations related to motor-goal representation in PRR. The directional selectivity could be preserved, erased or evoked by the pathway-selective optogenetic inhibition. In addition, the optogenetic modification increased the latency of motor-related tuning exclusively during the context-dependent sensorimotor transformations (i.e., when the task requires spatial remapping). These results support the hypothesis that dynamic reorganization in PRR is contingent on frontoparietal projections from PMd. 


\section{Neuronal and behavioral modulations by optogenetic stimulation}

In PMd, neurons expressing eArchT3.0 (hyperpolarizing opsins) were selectively inhibited by 532nm laser stimulation. In PRR, optical stimulation of eArchT3.0 expressed on axon terminals led to the inactivation of the presynaptic action potential. In this research, we found optogenetic inhibition of PMd axon terminals affected postsynaptic single-neuron spiking in PRR.

We estimated if the optogenetic manipulations in our approach were sufficient to affect primate behavior. Previous studies have proved the feasibility of using local (Fetsch et al., 2018) and pathway-selective (Inoue et al., 2015; Galvan et al., 2016) optogenetics to affect primate's behavior. In our optogenetic experiment, we only induced neuronal but not behavioral modulations in the rhesus monkey. We consider two reasons for explaining the missing behavioral modulations. First, given the larger size of macaque brains, the volume of tissue that should be involved in optogenetic modulation is far larger than we had reached by using a single optical fiber. Second, the time course over which optical stimulation could affect behavior is limited. In our experiment design, the interval between the laser stimulation and reach onset was too long to trigger any potential behavioral changes because the neural network would compensate for the changed neural activity after laser offset.

\section{Optogenetically induced heterogeneous responses in PRR}

Previous studies (Pesaran et al., 2008; Westendorff et al., 2010) proposed the hypothesis that the PMd-to-PRR projections play a functional role in motor-goal tuning in PRR, especially when the task required integrating contextual information with the sensory stimulus (spatial remapping) for correct motor goal selection. By inhibiting PMd-to-PRR projections, we could predict that PRR 
neurons partially lost their ability for encoding correct motor goals or showed delayed motor-goal tuning, exclusively when spatial remapping was required (i.e., in anti-reach). We tested the predictions and found that directional selectivity in PRR could be preserved, erased or evoked by the pathway-selective optogenetic inhibition. Given the fact that PMd-to-PRR projections are relatively sparse and the optical stimulation could only affect a limited area in brain tissue, it is reasonable to find a group of PRR neurons preserved their tuning properties after optogenetically inhibiting the projections. Another group of PRR neurons lost their tuning properties by the pathway-selective optogenetic inhibition, and the attenuating effects of light-inactivation on motor-goal encoding were significant. Importantly, this optogenetically induced attenuation was larger in anti-reach compared to pro-reach context, which is consistent with the predictions of the previous hypothesis that PMd-to-PRR projections dominantly function when the task required integrating contextual information with sensory stimulus for correct motor goal selection. Interestingly, we also observed a group of neurons without significant tuning in Non-Stim conditions but exhibited significant selectivity by light-inactivation. One hypothesis that could explain this observation is based on the compensation effect of the neural network: the network recruits neurons and endowing them with the ability to encode task-related variables for compensating the dysfunction of other neurons. The optogenetically induced heterogeneous responses and delaying effects in PRR provide a new insight for understanding the functional roles of premotor-to-parietal projections during context-dependent sensorimotor transformations.

\section{Optogenetically induced delaying effects on motor-goal tuning}

Optogenetics opens up causal investigation and specificity for the fast timescales of nervous system communication. By harnessing the temporal specificity of 
optogenetics, we found that PMd-to-PRR projections contribute to sensorimotor transformations during anti-reach task. The changed latency of motor-goal tuning while inhibiting the specific projections from PMd to PRR indicates whether the premotor-to-parietal projection serves to relatively enhance, or diminish inter-areal communication. Motor-goal encoding neurons in PRR showed attenuated and delayed responses to optogenetic inhibition of PMd projections in anti-reach contexts, while only showed attenuated responses in pro-reach contexts. The different delaying effects could be linked to the specific group of neurons which was characterized by a larger attenuating motor-goal representation in anti-reach trials. We interpret our results in support of the hypothesis that the direct projections from PMd to PRR causally affected neural responses during visuomotor transformations and motor goal encoding in PRR, especially when contextual information is involved. This study shows that top-down signals from PMd influence reach planning in the posterior parietal cortex.

\section{Figure captions:}

Figure 1. Behavioral task and experiment settings. (A) Timeline of the memoryguided center-out anti-reach task. The left sequence shows the pro-trial, the right is anti-trial. Red dots and grey disc in the center represent the eye- and hand-fixation area. The colored frame in the center represents the transformation rule (pro or anti), and the peripheral grey disc (at $0,90,180$, or 270 positions) represents the spatial cue. The dotted circle indicates the target position. Arrows and dotted circles during the movement period are for demonstration purpose only and were not shown to the monkeys. (B) The cortical locations for virus injection in PMd and putative PMd-to-PRR projections. (C) Extracellular recording in PMd, in 
combination with optogenetic silencing of the cell body, the top right panel shows the cortical locations in which recordings were conducted. The white dots represent the virus injection sites. (D) Extracellular recording in PRR, in combination with optogenetic silencing of synaptic terminals of PMd neurons expressing eArchT3.0, the top right panel shows the cortical locations at which recordings were conducted. (E) Optogenetic inhibition protocol. Continuous 532nm green laser covers visual cue and early phase of memory period.

Figure 2. Opsins expression and light-induced suppression of neuronal activity. (A) An example of the ChR2-eYFP transgene expression in the PMd in Monkey O (only available for histology); a $50 \mu \mathrm{m}$ thick coronal section from the area at the level of injections were immunoreacted against eYFP (in green) and Parvalbumin (in magenta). Effective transduction radius was $\sim 1-1.5 \mathrm{~mm}$ around needle tract; scale bar is shown. (B) A raster plot from an example neuron recorded in area PMd in Monkey A which was transduced with eArchT3.0-eYFP (hyperpolarizing opsins) and selectively inhibited by 532nm laser illumination. (C) An illustration of eYFP positive axonal projections terminating in parietal cortex in Monkey O (only available for histology); a $50 \mu \mathrm{m}$ thick coronal section from the parietal cortex immunoreacted against eYFP (in green) and Parvalbumin (in red) and stained with DAPI (blue); an inset for anatomical orientation adopted from the 'Atlas of the Rhesus Monkey Brain in Stereotaxic Coordinates' by Saleem KS and Logothetis NK. An expanded view of the area MIP is in the box; scale bar is shown. (D) A raster plot from an example neuron recorded in MIP (Monkey A); the neuron was slightly inhibited by 532nm laser illumination. Abbreviations: ChR2 - Channelrhodopsin-2, eYFP - enhanced yellow fluorescent protein, PV Parvalbumin, PMdc - caudal-dorsal premotor cortex, PMv - ventral premotor 
cortex, arsp - spur of the arcuate sulcus, MIP - medial interparietal area. Panels A and $\mathrm{C}$ were provided by Dr. Michal Fortuna.

Figure 3. Dynamics of motor-related tuning of neurons in the "preserved” group. (A-B) Data (“preserved” group) for pro-trials (A, green) and anti-trials (B, blue) are aligned to the onset of the visual cue. The analysis time window is between 200 ms before and $700 \mathrm{~ms}$ after the onset of the visual cue. Top left table shows the number of neurons in each categorization (see Materials and Methods).

Figure 4. Dynamics of motor-related tuning of neurons in the "erased" and “evoked” groups. (A-B) Data (“erased” group) for pro-trials (A, green) and antitrials (B, blue) are aligned to the onset of the visual cue. (C-D) Data ("evoked" group) for pro-trials (A, green) and anti-trials (B, blue) aligned to the onset of the visual cue. The black bars on the top indicate the time at which the neural response to the MD under Non-Stim and Opto-Stim are significantly different.

Figure 5. Effect of optogenetic inhibition on motor-goal latency. (A-B) The latency of motor tuning within each condition is defined as the time at which the neural responses to the MD and NP are significantly different. Two vertical dashed lines indicate the significant separation time (permutation test, $\mathrm{p}<0.05$ ) in NonStim (black) and Opto-Stim (grey) conditions. The latency difference between Non-Stim and Opto-Stim condition was tested with a permutation test (see Materials and Methods). 


\section{Figure 1}

A

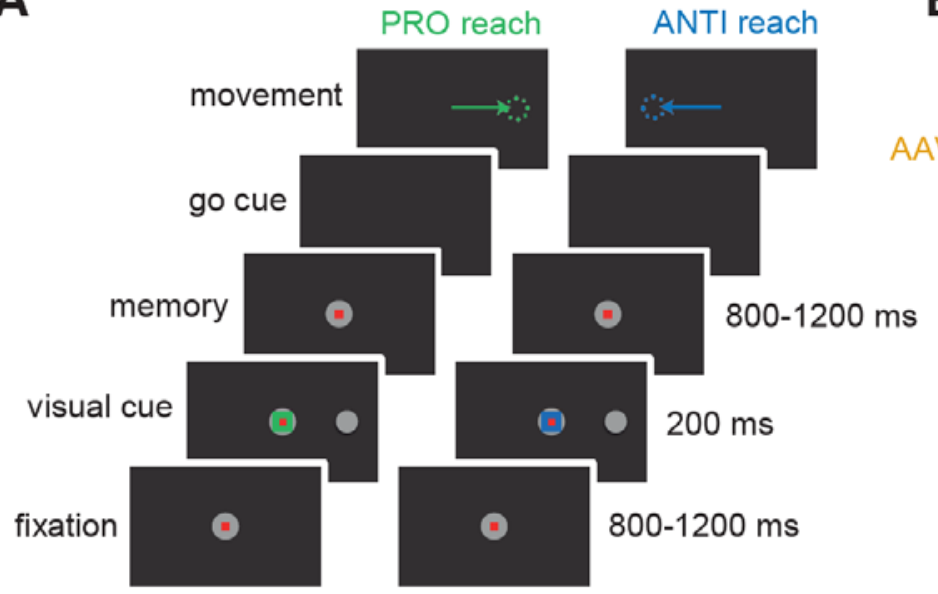

B

Virus injections in PMd

AAV2/5-CaMKIla-eArchT3.0-eYFP PRR

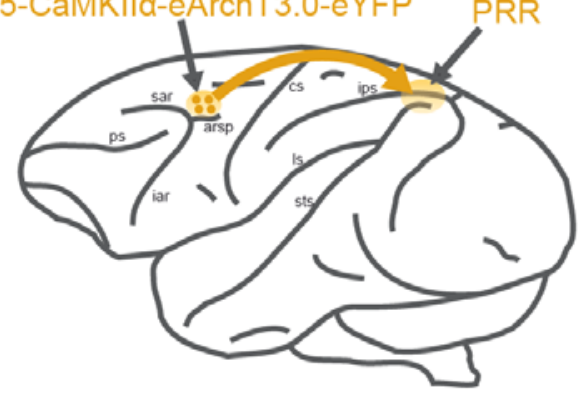

C

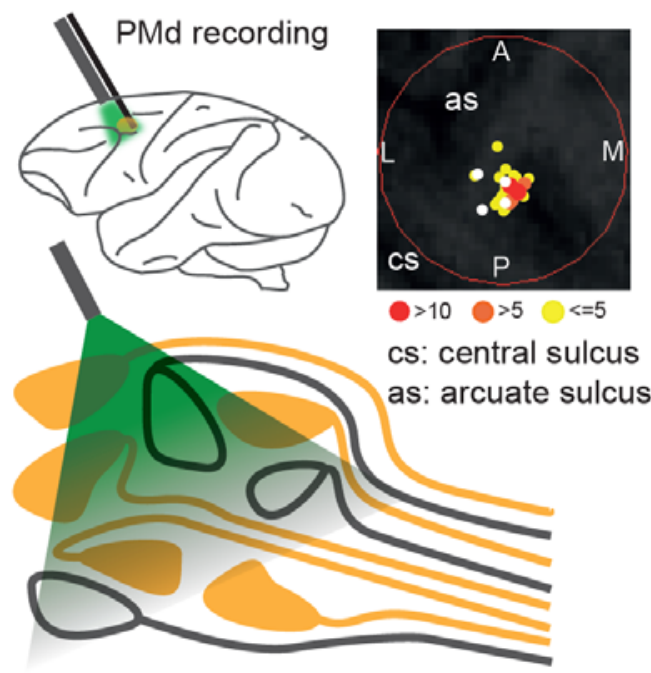

Neuros expressing eArchT3.0

Neuros not expressing eArchT3.0

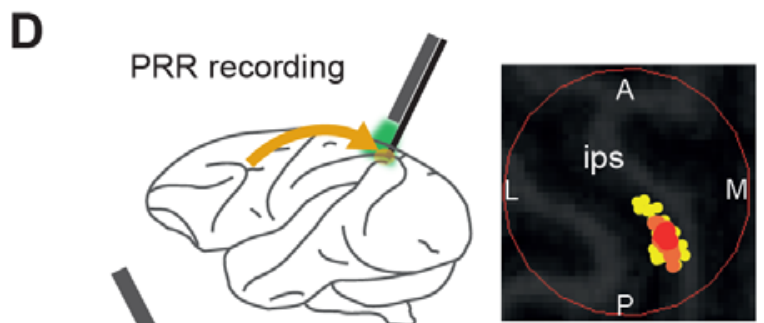

ips: intraparietal sulcus

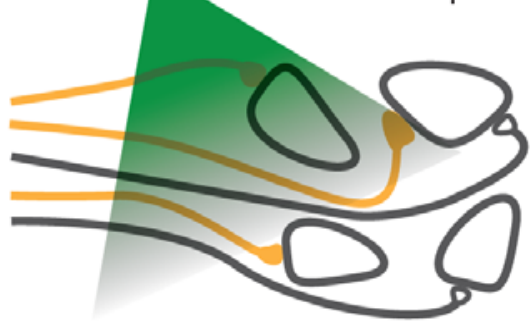

Axon terminals expressing eArchT3.0

Axon terminals not expressing eArchT3.0

E

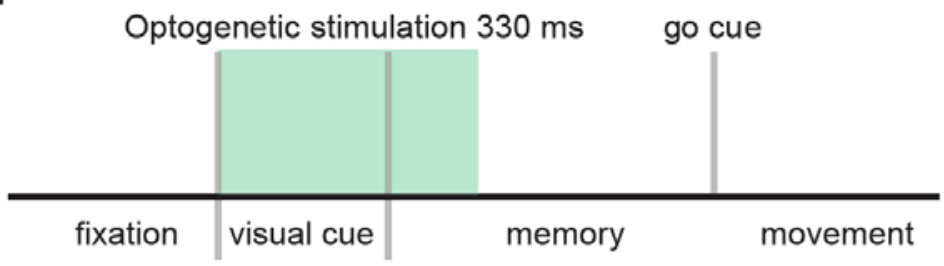


Figure 2

A

Monkey $O$

ChR2-eYFP

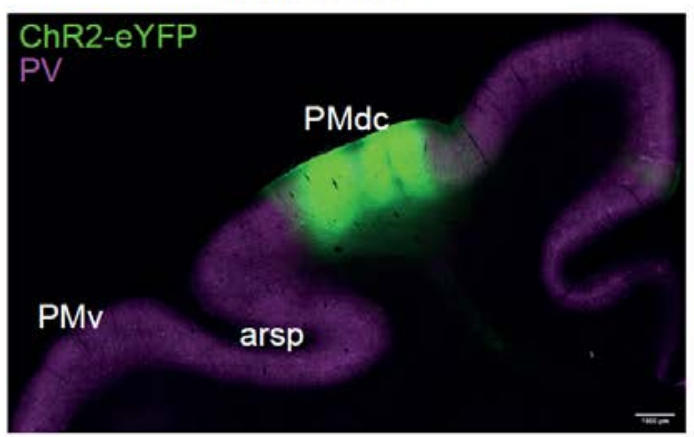

C

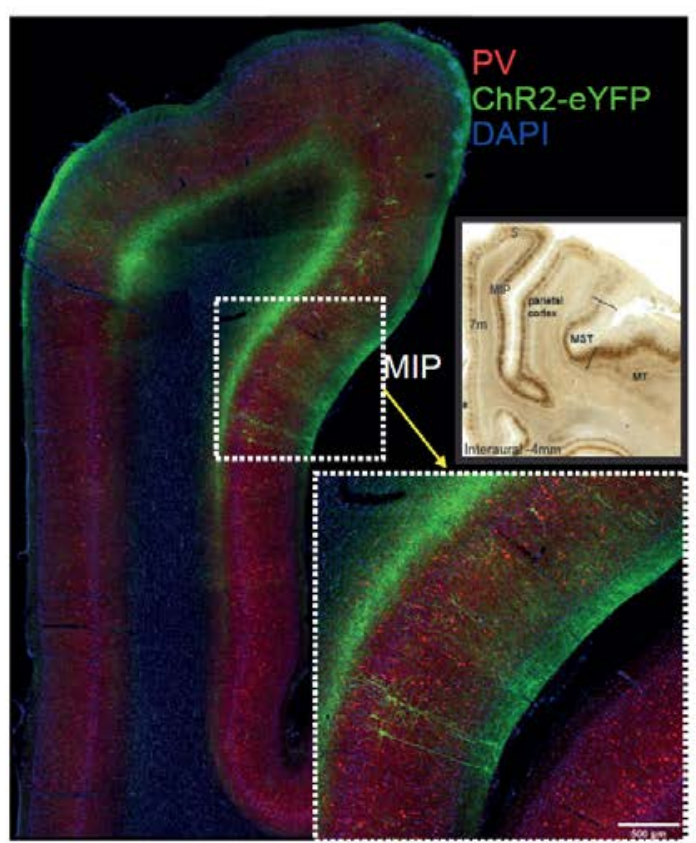

B

Monkey A

eArchT3.0-eYFP

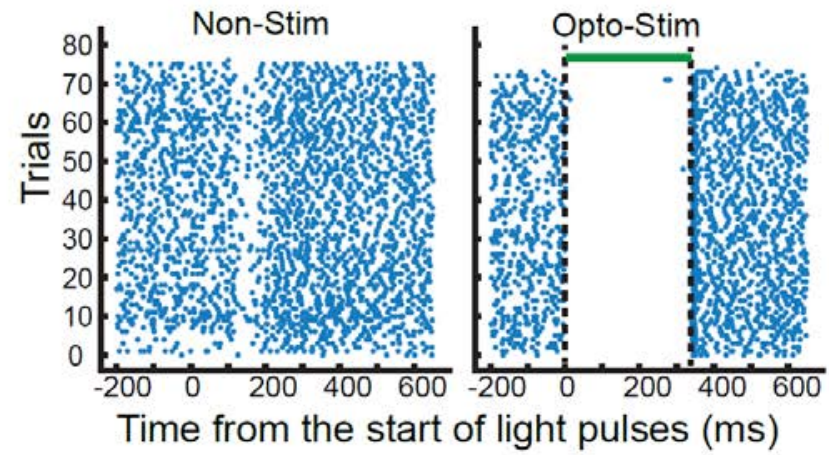

D Non-Stim Opto-Stim

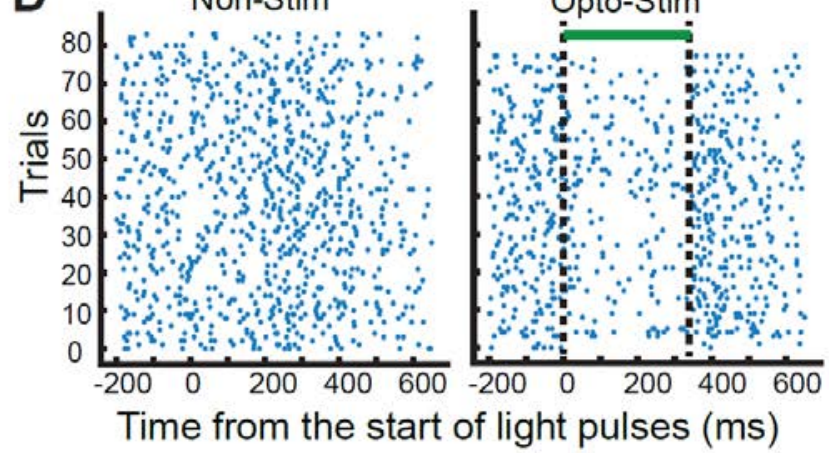


Figure 3
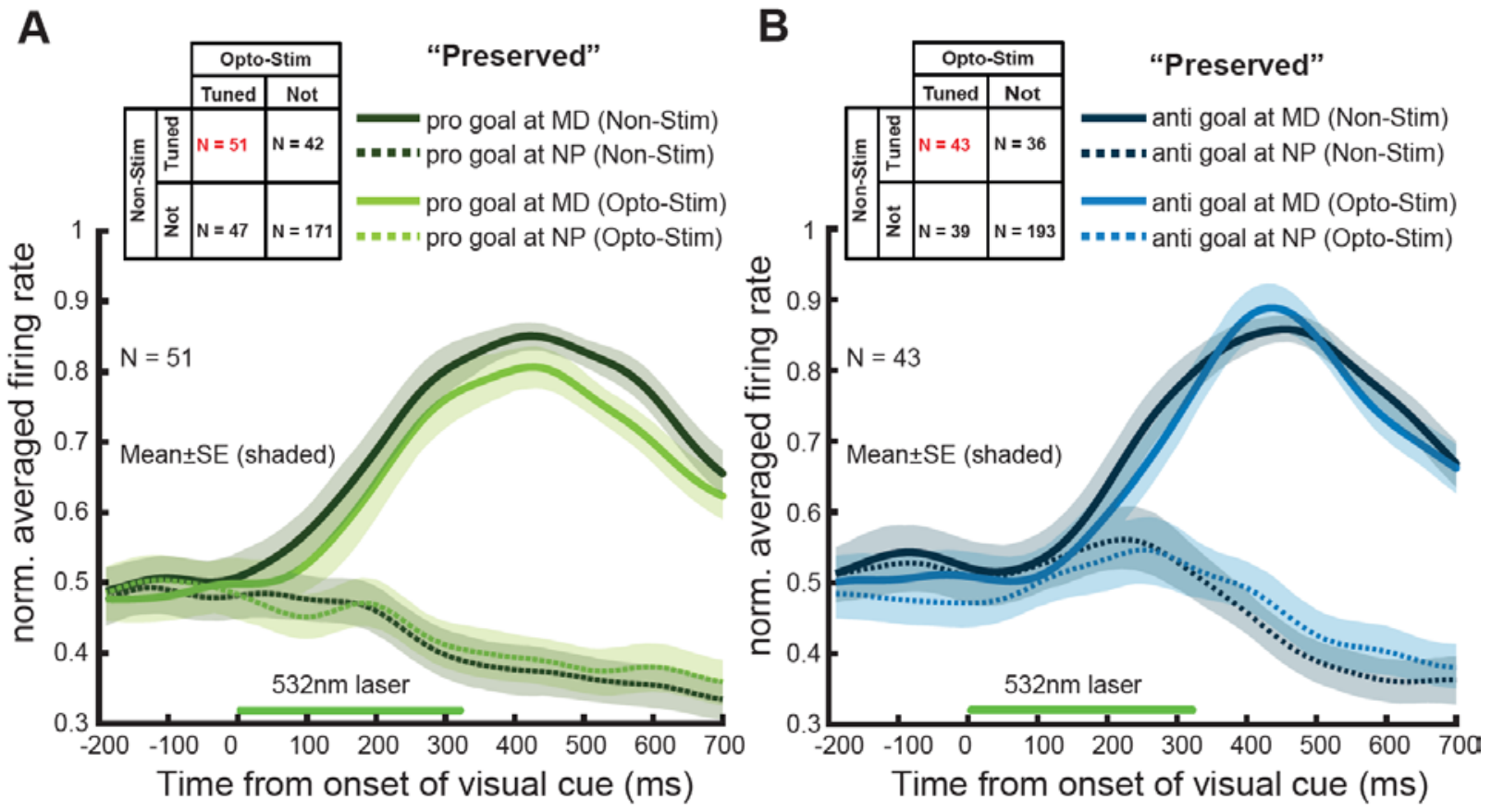
Figure 4

A

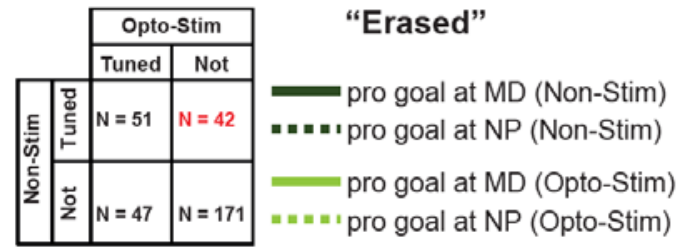

B

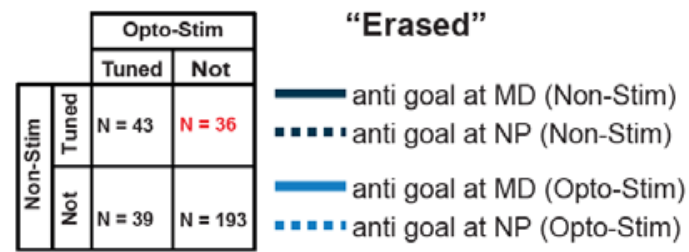

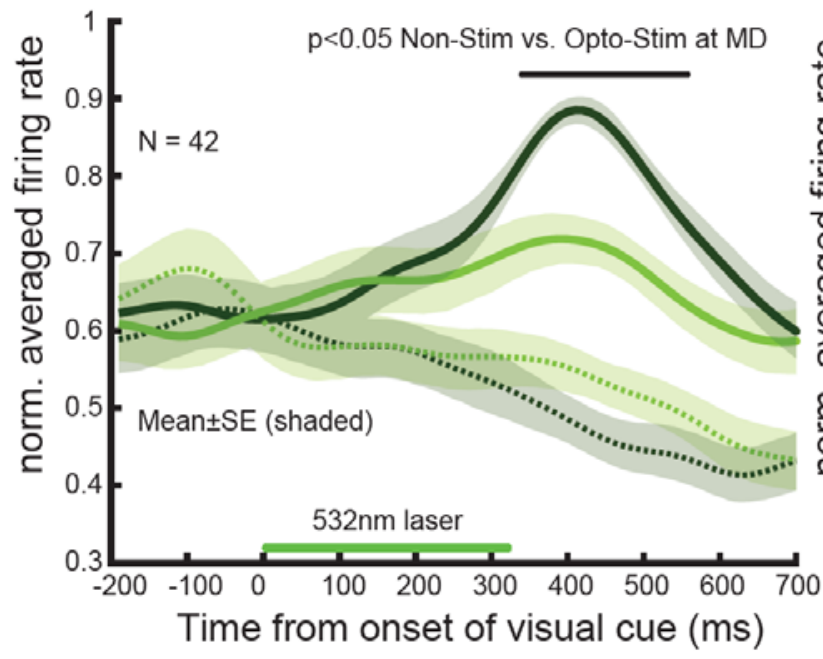

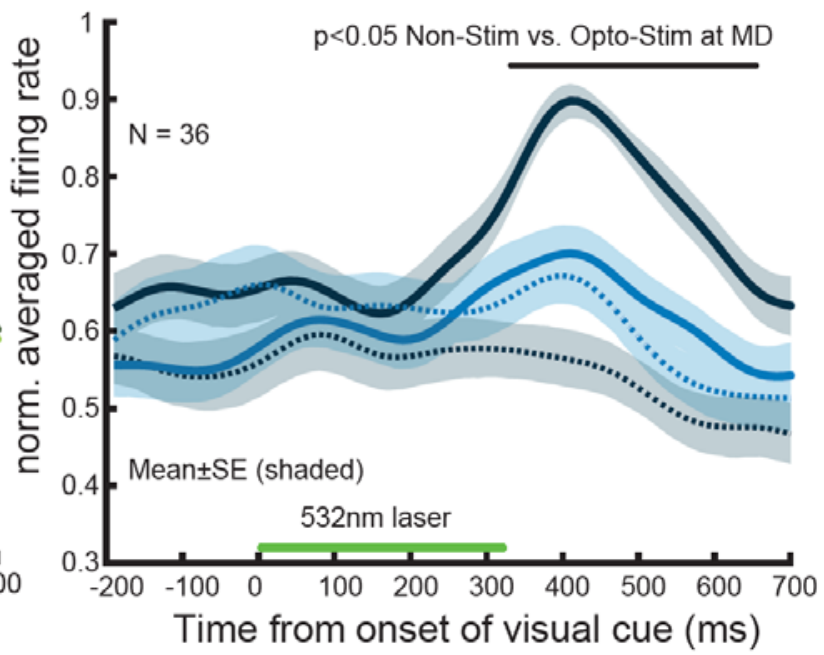

C
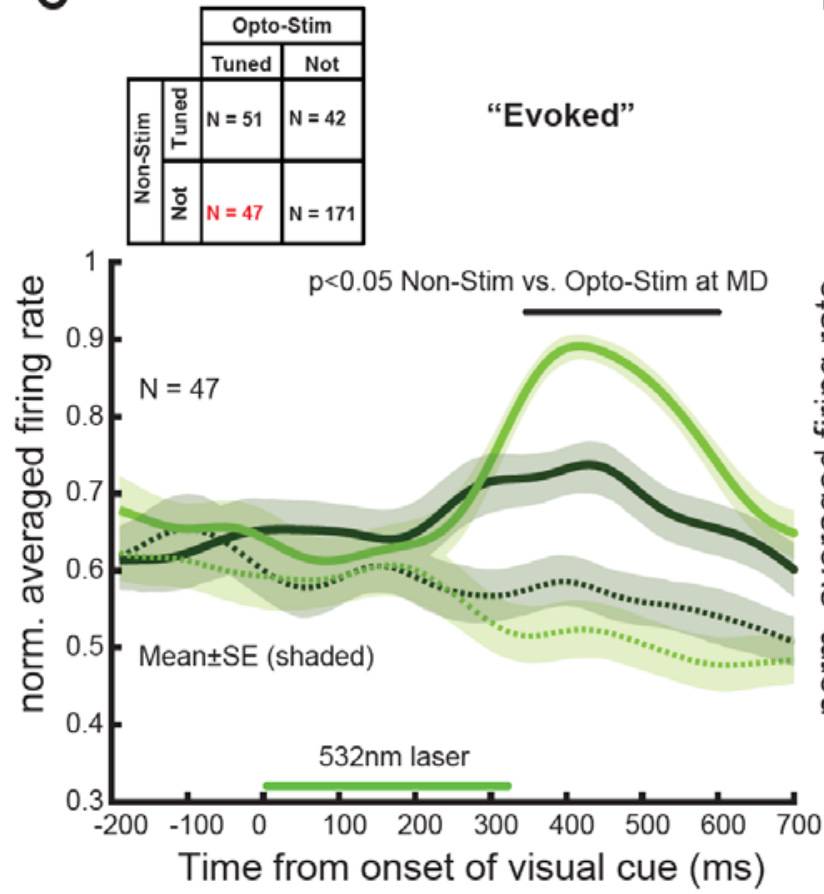

"Evoked"
D

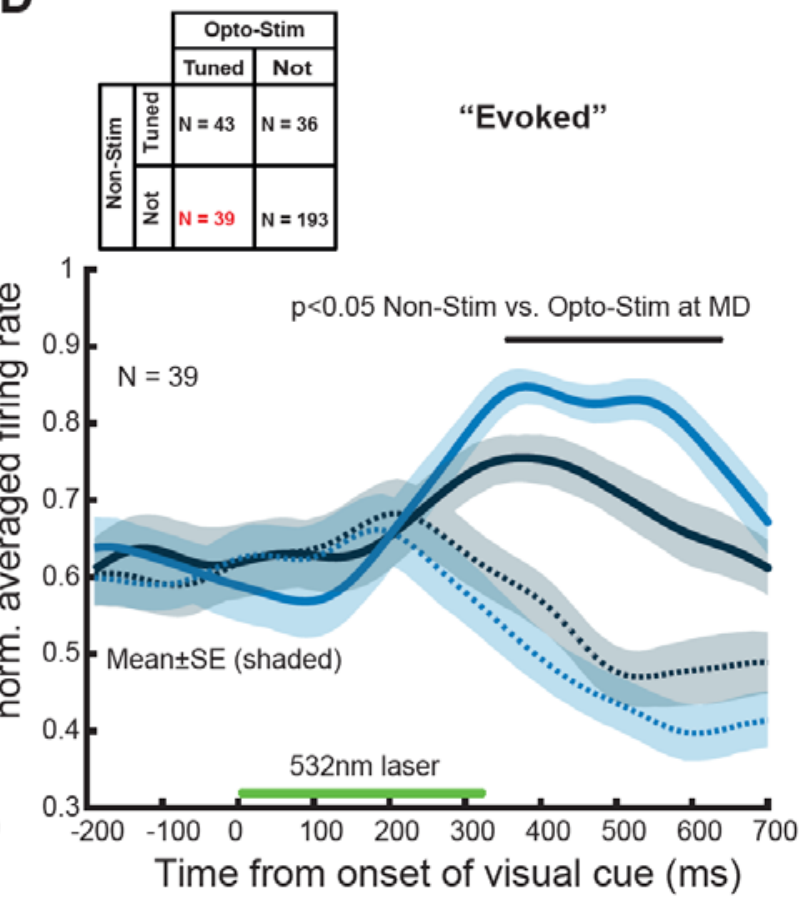


Figure 5
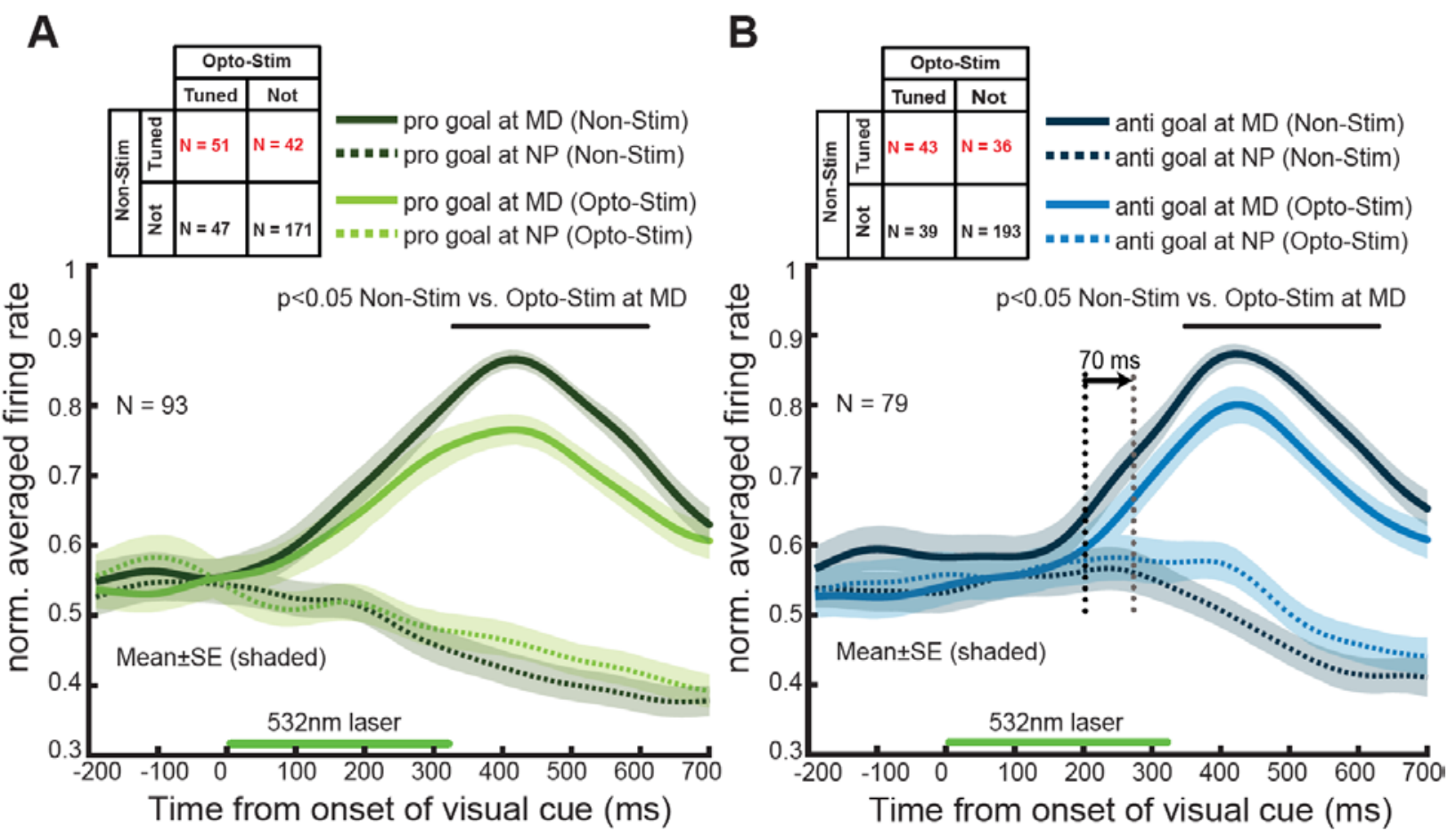


\section{References}

Bakola S, Gamberini M, Passarelli L, Fattori P, Galletti C (2010) Cortical connections of parietal field PEc in the macaque: linking vision and somatic sensation for the control of limb action. Cerebral cortex (New York, NY : 1991) 20:2592-2604.

Brozovic M, Gail A, Andersen RA (2007) Gain mechanisms for contextually guided visuomotor transformations. J Neurosci 27:10588-10596.

Caminiti R, Genovesio A, Marconi B, Mayer AB, Onorati P, Ferraina S, Mitsuda T, Giannetti S, Squatrito S, Maioli MG, Molinari M (1999) Early coding of reaching: frontal and parietal association connections of parieto-occipital cortex. Eur J Neurosci 11:3339-3345.

Cisek P (2007) Cortical mechanisms of action selection: the affordance competition hypothesis. Philos Trans R Soc Lond B Biol Sci 362:1585-1599.

Crammond DJ, Kalaska JF (1994) Modulation of preparatory neuronal activity in dorsal premotor cortex due to stimulus-response compatibility. Journal of neurophysiology 71:1281-1284.

Deisseroth K (2015) Optogenetics: 10 years of microbial opsins in neuroscience. Nat Neurosci 18:1213-1225.

Fetsch CR, Odean NN, Jeurissen D, El-Shamayleh Y, Horwitz GD, Shadlen MN (2018) Focal optogenetic suppression in macaque area MT biases direction discrimination and decision confidence, but only transiently. eLife 7.

Gail A, Andersen RA (2006) Neural dynamics in monkey parietal reach region reflect context-specific sensorimotor transformations. J Neurosci 26:9376-9384.

Gail A, Klaes C, Westendorff S (2009) Implementation of spatial transformation rules for goal-directed reaching via gain modulation in monkey parietal and premotor cortex. J Neurosci 29:9490-9499.

Galvan A, Hu X, Smith Y, Wichmann T (2016) Effects of Optogenetic Activation of Corticothalamic Terminals in the Motor Thalamus of Awake Monkeys. J Neurosci 36:3519-3530.

Galvan A, Stauffer WR, Acker L, El-Shamayleh Y, Inoue KI, Ohayon S, Schmid MC (2017) Nonhuman Primate Optogenetics: Recent Advances and Future Directions. J Neurosci 37:10894-10903. 
Gamberini M, Passarelli L, Fattori P, Zucchelli M, Bakola S, Luppino G, Galletti C (2009) Cortical connections of the visuomotor parietooccipital area V6Ad of the macaque monkey. J Comp Neurol 513:622-642.

Grosenick L, Marshel JH, Deisseroth K (2015) Closed-loop and activity-guided optogenetic control. Neuron 86:106-139.

Han X, Chow BY, Zhou H, Klapoetke NC, Chuong A, Rajimehr R, Yang A, Baratta MV, Winkle J, Desimone R, Boyden ES (2011) A high-light sensitivity optical neural silencer: development and application to optogenetic control of nonhuman primate cortex. Front Syst Neurosci 5:18.

Inoue K, Takada M, Matsumoto M (2015) Neuronal and behavioural modulations by pathway-selective optogenetic stimulation of the primate oculomotor system. Nat Commun 6:8378.

Johnson PB, Ferraina S, Bianchi L, Caminiti R (1996) Cortical networks for visual reaching: physiological and anatomical organization of frontal and parietal lobe arm regions. Cerebral cortex (New York, NY : 1991) 6:102-119.

Luppino G, Calzavara R, Rozzi S, Matelli M (2001) Projections from the superior temporal sulcus to the agranular frontal cortex in the macaque. Eur J Neurosci 14:1035-1040.

Luppino G, Rozzi S, Calzavara R, Matelli M (2003) Prefrontal and agranular cingulate projections to the dorsal premotor areas F2 and F7 in the macaque monkey. Eur J Neurosci 17:559-578.

Marconi B, Genovesio A, Battaglia-Mayer A, Ferraina S, Squatrito S, Molinari M, Lacquaniti F, Caminiti R (2001) Eye-hand coordination during reaching. I. Anatomical relationships between parietal and frontal cortex. Cerebral cortex (New York, NY : 1991) 11:513-527.

Markov NT et al. (2014) A weighted and directed interareal connectivity matrix for macaque cerebral cortex. Cerebral cortex (New York, NY : 1991) 24:17-36.

Martinez-Vazquez P, Gail A (2018) Directed Interaction Between Monkey Premotor and Posterior Parietal Cortex During Motor-Goal Retrieval from Working Memory. Cerebral cortex (New York, NY : 1991) 28:1866-1881.

Matelli M, Govoni P, Galletti C, Kutz DF, Luppino G (1998) Superior area 6 afferents from the superior parietal lobule in the macaque monkey. J Comp Neurol 402:327-352.

Passarelli L, Rosa MG, Gamberini M, Bakola S, Burman KJ, Fattori P, Galletti C (2011) Cortical connections of area V6Av in the macaque: a visual-input node to the eye/hand coordination system. J Neurosci 31:1790-1801. 
Pesaran B, Nelson MJ, Andersen RA (2008) Free choice activates a decision circuit between frontal and parietal cortex. Nature 453:406-409.

Petrides M, Pandya DN (1999) Dorsolateral prefrontal cortex: comparative cytoarchitectonic analysis in the human and the macaque brain and corticocortical connection patterns. Eur J Neurosci 11:1011-1036.

Stuber GD, Sparta DR, Stamatakis AM, van Leeuwen WA, Hardjoprajitno JE, Cho S, Tye KM, Kempadoo KA, Zhang F, Deisseroth K, Bonci A (2011) Excitatory transmission from the amygdala to nucleus accumbens facilitates reward seeking. Nature 475:377-380.

Tanne-Gariepy J, Rouiller EM, Boussaoud D (2002) Parietal inputs to dorsal versus ventral premotor areas in the macaque monkey: evidence for largely segregated visuomotor pathways. Exp Brain Res 145:91-103.

Tye KM, Prakash R, Kim SY, Fenno LE, Grosenick L, Zarabi H, Thompson KR, Gradinaru V, Ramakrishnan C, Deisseroth K (2011) Amygdala circuitry mediating reversible and bidirectional control of anxiety. Nature 471:358-362.

Wallis JD, Miller EK (2003) From rule to response: neuronal processes in the premotor and prefrontal cortex. Journal of neurophysiology 90:1790-1806.

Warden MR, Selimbeyoglu A, Mirzabekov JJ, Lo M, Thompson KR, Kim SY, Adhikari A, Tye KM, Frank LM, Deisseroth K (2012) A prefrontal cortexbrainstem neuronal projection that controls response to behavioural challenge. Nature 492:428-432.

Westendorff S, Klaes C, Gail A (2010) The cortical timeline for deciding on reach motor goals. J Neurosci 30:5426-5436. 


\section{3 - General Discussion}

In summary, the work presented in this dissertation applied state-space analyses and optogenetic approaches to investigate the frontoparietal reach network in rhesus monkeys, yielding three important findings to the current understandings about the neural mechanisms underlying spatial representations and sensorimotor transformations of goal-directed reaching movements.

\section{Data analyses based on the framework of dynamical process}

In chapter 2.1, the neurophysiological results added new perspectives to the functional differences between distinct areas in the frontoparietal network of rhesus monkey. PMd and PRR exhibited similar encoding of the anticipated visual sensory consequences of intended movement but different neuronal dynamics during the planning-to-execution transition. Specifically, the state-space analysis provided a quantitative and more meaningful interpretation for the functional differences between frontoparietal areas. From the results of this analysis, we observed PMd showed a larger neural heterogeneity and dynamics, whereas, PRR was endowed with a more stable and robust dynamics from planning to movement.

In chapter 2.2, we investigated the computational strategies that are exploited by the brain for context-dependent sensorimotor transformation. By applying statespace analyses, we found computational strategies, which are confined to the specific neural subspaces, differed under distinct contextual configurations. Furthermore, when the contextual information was introduced into the neural system by applying a reversed-viewing prism (Kuang et al., 2016), the contextual information affected cortical dynamics by systematically altering baseline neural activity, corresponding to a shifted initial condition in the dynamical system. 
These results demonstrated a simple structure in the neural population response and potentially provided a general framework for understanding cognitive computation. There are growing bodies of experimental data that are difficult to investigate from a single-neuron level but become more approachable when dynamical systems concepts (Cunningham and Yu, 2014; Gallego et al., 2017) are used. When behavioral demands differed, different computations could be implemented in the same neural population (Raposo et al., 2014). A previous study revealed that when behavioral demands evolved from preparatory to movement, PMd exploited an orthogonal population-level subspace (Elsayed et al., 2016). Similar results were also found in the posterior parietal cortex of rodents (Raposo et al., 2014). Our studies furthered the understanding of neural computations by comparing the dynamics in PMd and PRR and introducing the context-specific sensorimotor computation as a new paradigm. Together, these findings could be interpreted by the "neural manifold” concept (Sadtler et al., 2014; Gao and Ganguli, 2015; Gallego et al., 2017) which has been used to explain experimental data in multiple brain areas across a variety of paradigms (Stopfer et al., 2003; Churchland et al., 2010; Churchland et al., 2012; Harvey et al., 2012). It is worth noting that previous theoretical studies have mostly focused on modeling the sensorimotor transformations on single-neuron level (Brozovic et al., 2007), or based on neural field model (Klaes et al., 2012), which both failed to link to the "neural manifold" theory because it is defined only at the level of the neural population. A recent modeling work based on a recurrent neural network mainly focused on the movement generation process (Sussillo et al., 2015). Thus, further extensions of modeling studies would be of great interest to our current understanding of neural computations. 


\section{Optogenetics experiment on monkeys}

In chapter 2.3, we developed and applied the optogenetic approach for the monkey neurophysiology experiment. Our approach has been proved not only efficient for manipulating single-neuron activity, but also functional for exerting influence on specific inter-areal neural projections. The previous hypothesis addressed that the information flows from PMd to PRR are functional in rule-based sensorimotor computations (Pesaran et al., 2008; Westendorff et al., 2010). By optogenetically silencing PMd-to-PRR projections, we directly tested whether the dynamic reorganization of network activity in PRR is contingent on the projections from PMd. We recorded the activity of single neurons from PRR in combination with pathway-selective optogenetic inhibition, while a rhesus monkey performed a rulebased center-out reach task. Optogenetic inhibition of PMd-to-PRR projections resulted in heterogeneous neural modulations in PRR. The directional selectivity of PRR neurons could be preserved, erased or evoked by optical stimulation. To our knowledge, this is the first neuronal evidence that clearly shows single-neuron activity in the posterior parietal cortex is causally affected by the inputs from frontal lobe. In recent years, optogenetics has offered great potential for investigating brain circuits and linking brain function and behavior in non-human primates (Ruiz et al., 2013; Afraz et al., 2015; Inoue et al., 2015; Nassi et al., 2015; Acker et al., 2016; Galvan et al., 2016; Stauffer et al., 2016; El-Shamayleh et al., 2017; Tamura et al., 2017; Fetsch et al., 2018). Our results illustrated a role of output from PMd to its downstream structure PRR that could be exploited for context-dependent visuomotor transformation, and provided the direct evidence for the long-lasting debate about the mutual interaction and coordination in the frontoparietal network (Pesaran et al., 2008; Westendorff et al., 2010; Stetson and Andersen, 2014; Martinez-Vazquez and Gail, 2018). 
In conclusion, this dissertation provided both computational and neuronal evidence emphasizing flexible and dynamic sensorimotor transformations within the frontoparietal reach network. It systematically investigated the neural population dynamics and frontal-to-parietal information stream during context-dependent sensorimotor computations, and provided novel perspectives on the function of frontoparietal reach network. 


\section{4 - Bibliography}

Acker L, Pino EN, Boyden ES, Desimone R (2016) FEF inactivation with improved optogenetic methods. Proceedings of the National Academy of Sciences of the United States of America 113:E7297-E7306.

Adhikari A, Lerner TN, Finkelstein J, Pak S, Jennings JH, Davidson TJ, Ferenczi E, Gunaydin LA, Mirzabekov JJ, Ye L, Kim SY, Lei A, Deisseroth K (2015) Basomedial amygdala mediates top-down control of anxiety and fear. Nature 527:179-185.

Afraz A, Boyden ES, DiCarlo JJ (2015) Optogenetic and pharmacological suppression of spatial clusters of face neurons reveal their causal role in face gender discrimination. Proceedings of the National Academy of Sciences of the United States of America 112:6730-6735.

Afshar A, Santhanam G, Yu BM, Ryu SI, Sahani M, Shenoy KV (2011) Singletrial neural correlates of arm movement preparation. Neuron 71:555-564.

Ames KC, Ryu SI, Shenoy KV (2014) Neural dynamics of reaching following incorrect or absent motor preparation. Neuron 81:438-451.

Andersen RA, Buneo CA (2002) Intentional maps in posterior parietal cortex. Annu Rev Neurosci 25:189-220.

Andersen RA, Cui H (2009) Intention, action planning, and decision making in parietal-frontal circuits. Neuron 63:568-583.

Archambault PS, Ferrari-Toniolo S, Battaglia-Mayer A (2011) Online control of hand trajectory and evolution of motor intention in the parietofrontal system. The Journal of neuroscience : the official journal of the Society for Neuroscience 31:742-752.

Asaad WF, Rainer G, Miller EK (1998) Neural activity in the primate prefrontal cortex during associative learning. Neuron 21:1399-1407.

Bakker R, Wachtler T, Diesmann M (2012) CoCoMac 2.0 and the future of tracttracing databases. Frontiers in neuroinformatics 6:30.

Bakola S, Gamberini M, Passarelli L, Fattori P, Galletti C (2010) Cortical connections of parietal field PEc in the macaque: linking vision and somatic sensation for the control of limb action. Cerebral cortex (New York, NY : 1991) 20:2592-2604.

Barbas H, Pandya DN (1987) Architecture and frontal cortical connections of the premotor cortex (area 6) in the rhesus monkey. J Comp Neurol 256:211-228. 
Barbas H, Pandya DN (1989) Architecture and intrinsic connections of the prefrontal cortex in the rhesus monkey. J Comp Neurol 286:353-375.

Batista AP, Buneo CA, Snyder LH, Andersen RA (1999) Reach plans in eyecentered coordinates. Science 285:257-260.

Batista AP, Santhanam G, Yu BM, Ryu SI, Afshar A, Shenoy KV (2007) Reference frames for reach planning in macaque dorsal premotor cortex. Journal of neurophysiology 98:966-983.

Battaglia-Mayer A, Caminiti R, Lacquaniti F, Zago M (2003) Multiple levels of representation of reaching in the parieto-frontal network. Cereb Cortex 13:10091022.

Bosco A, Breveglieri R, Chinellato E, Galletti C, Fattori P (2010) Reaching activity in the medial posterior parietal cortex of monkeys is modulated by visual feedback. Journal of Neuroscience 30:14773-14785.

Boyden ES, Zhang F, Bamberg E, Nagel G, Deisseroth K (2005) Millisecondtimescale, genetically targeted optical control of neural activity. Nat Neurosci 8:1263-1268.

Bremner LR, Andersen RA (2014) Temporal analysis of reference frames in parietal cortex area $5 d$ during reach planning. The Journal of neuroscience : the official journal of the Society for Neuroscience 34:5273-5284.

Brozovic M, Gail A, Andersen RA (2007) Gain mechanisms for contextually guided visuomotor transformations. J Neurosci 27:10588-10596.

Buneo CA, Jarvis MR, Batista AP, Andersen RA (2002) Direct visuomotor transformations for reaching. Nature 416:632-636.

Buneo CA, Batista AP, Jarvis MR, Andersen RA (2008) Time-invariant reference frames for parietal reach activity. Experimental brain research 188:77-89.

Caminiti R, Zeger S, Johnson PB, Urbano A, Georgopoulos AP (1985) Corticocortical efferent systems in the monkey: a quantitative spatial analysis of the tangential distribution of cells of origin. J Comp Neurol 241:405-419.

Caminiti R, Borra E, Visco-Comandini F, Battaglia-Mayer A, Averbeck BB, Luppino G (2017) Computational Architecture of the Parieto-Frontal Network Underlying Cognitive-Motor Control in Monkeys. eNeuro 4.

Caminiti R, Genovesio A, Marconi B, Mayer AB, Onorati P, Ferraina S, Mitsuda T, Giannetti S, Squatrito S, Maioli MG, Molinari M (1999) Early coding of reaching: frontal and parietal association connections of parieto-occipital cortex. Eur J Neurosci 11:3339-3345. 
Cavanaugh J, Monosov IE, McAlonan K, Berman R, Smith MK, Cao V, Wang KH, Boyden ES, Wurtz RH (2012) Optogenetic inactivation modifies monkey visuomotor behavior. Neuron 76:901-907.

Chakrabarti S, Martinez-Vazquez P, Gail A (2014) Synchronization patterns suggest different functional organization in parietal reach region and dorsal premotor cortex. Journal of neurophysiology 112:3138-3153.

Chang SW, Snyder LH (2010) Idiosyncratic and systematic aspects of spatial representations in the macaque parietal cortex. ProcNatlAcadSciUSA 107:79517956.

Chow BY, Han X, Dobry AS, Qian X, Chuong AS, Li M, Henninger MA, Belfort GM, Lin Y, Monahan PE, Boyden ES (2010) High-performance genetically targetable optical neural silencing by light-driven proton pumps. Nature 463:98102.

Churchland MM, Shenoy KV (2007) Temporal complexity and heterogeneity of single-neuron activity in premotor and motor cortex. Journal of Neurophysiology 97:4235-4257.

Churchland MM, Cunningham JP, Kaufman MT, Ryu SI, Shenoy KV (2010) Cortical preparatory activity: representation of movement or first cog in a dynamical machine? Neuron 68:387-400.

Churchland MM, Cunningham JP, Kaufman MT, Foster JD, Nuyujukian P, Ryu SI, Shenoy KV (2012) Neural population dynamics during reaching. Nature 487:51-56.

Cisek P (2006) Integrated neural processes for defining potential actions and deciding between them: a computational model. J Neurosci 26:9761-9770.

Cisek P (2007) Cortical mechanisms of action selection: the affordance competition hypothesis. Philos Trans R Soc Lond B Biol Sci 362:1585-1599.

Cisek P, Kalaska JF (2010) Neural mechanisms for interacting with a world full of action choices. Annu Rev Neurosci 33:269-298.

Cohen YE, Andersen RA (2002) A common reference frame for movement plans in the posterior parietal cortex. Nat Rev Neurosci 3:553-562.

Colby CL, Gattass R, Olson CR, Gross CG (1988) Topographical organization of cortical afferents to extrastriate visual area PO in the macaque: a dual tracer study. J Comp Neurol 269:392-413.

Crammond DJ, Kalaska JF (1994) Modulation of preparatory neuronal activity in dorsal premotor cortex due to stimulus-response compatibility. Journal of neurophysiology 71:1281-1284. 
Crawford JD, Henriques DY, Medendorp WP (2011) Three-dimensional transformations for goal-directed action. Annual Review of Neuroscience 34:309331.

Cui H, Andersen RA (2007) Posterior parietal cortex encodes autonomously selected motor plans. Neuron 56:552-559.

Cui H, Andersen RA (2011) Different representations of potential and selected motor plans by distinct parietal areas. J Neurosci 31:18130-18136.

Cunningham JP, Yu BM (2014) Dimensionality reduction for large-scale neural recordings. Nat Neurosci 17:1500-1509.

Deisseroth K (2015) Optogenetics: 10 years of microbial opsins in neuroscience. Nat Neurosci 18:1213-1225.

Desmurget M, Sirigu A (2009) A parietal-premotor network for movement intention and motor awareness. Trends Cogn Sci 13:411-419.

Desmurget M, Reilly KT, Richard N, Szathmari A, Mottolese C, Sirigu A (2009) Movement intention after parietal cortex stimulation in humans. Science 324:811813.

Diester I, Kaufman MT, Mogri M, Pashaie R, Goo W, Yizhar O, Ramakrishnan C, Deisseroth K, Shenoy KV (2011) An optogenetic toolbox designed for primates. Nat Neurosci 14:387-397.

Dimidschstein J et al. (2016) A viral strategy for targeting and manipulating interneurons across vertebrate species. Nat Neurosci 19:1743-1749.

Duhamel JR, Colby CL, Goldberg ME (1992) The updating of the representation of visual space in parietal cortex by intended eye movements. Science 255:90-92.

El-Shamayleh Y, Kojima Y, Soetedjo R, Horwitz GD (2017) Selective Optogenetic Control of Purkinje Cells in Monkey Cerebellum. Neuron 95:51-62 e54.

Elsayed GF, Lara AH, Kaufman MT, Churchland MM, Cunningham JP (2016) Reorganization between preparatory and movement population responses in motor cortex. Nat Commun 7:13239.

Even-Chen N, Sheffer B, Vyas S, Ryu SI, Shenoy KV (2019) Structure and variability of delay activity in premotor cortex. PLoS Comput Biol 15:e1006808.

Everling S, DeSouza JF (2005) Rule-dependent activity for prosaccades and antisaccades in the primate prefrontal cortex. J Cogn Neurosci 17:1483-1496.

Felleman DJ, Van Essen DC (1991) Distributed hierarchical processing in the primate cerebral cortex. Cerebral cortex (New York, NY : 1991) 1:1-47. 
Fetsch CR, Odean NN, Jeurissen D, El-Shamayleh Y, Horwitz GD, Shadlen MN (2018) Focal optogenetic suppression in macaque area MT biases direction discrimination and decision confidence, but only transiently. eLife 7.

Franklin DW, Wolpert DM (2011) Computational mechanisms of sensorimotor control. Neuron 72:425-442.

Gail A, Andersen RA (2006) Neural dynamics in monkey parietal reach region reflect context-specific sensorimotor transformations. J Neurosci 26:9376-9384.

Gail A, Klaes C, Westendorff S (2009) Implementation of spatial transformation rules for goal-directed reaching via gain modulation in monkey parietal and premotor cortex. J Neurosci 29:9490-9499.

Gallego JA, Perich MG, Miller LE, Solla SA (2017) Neural Manifolds for the Control of Movement. Neuron 94:978-984.

Galvan A, Hu X, Smith Y, Wichmann T (2016) Effects of Optogenetic Activation of Corticothalamic Terminals in the Motor Thalamus of Awake Monkeys. J Neurosci 36:3519-3530.

Galvan A, Stauffer WR, Acker L, El-Shamayleh Y, Inoue KI, Ohayon S, Schmid MC (2017) Nonhuman Primate Optogenetics: Recent Advances and Future Directions. J Neurosci 37:10894-10903.

Gamberini M, Passarelli L, Fattori P, Zucchelli M, Bakola S, Luppino G, Galletti C (2009) Cortical connections of the visuomotor parietooccipital area V6Ad of the macaque monkey. J Comp Neurol 513:622-642.

Ganguli S, Bisley JW, Roitman JD, Shadlen MN, Goldberg ME, Miller KD (2008) One-dimensional dynamics of attention and decision making in LIP. Neuron 58:15-25.

Gao P, Ganguli S (2015) On simplicity and complexity in the brave new world of large-scale neuroscience. Curr Opin Neurobiol 32:148-155.

Gerits A, Vanduffel W (2013) Optogenetics in primates: a shining future? Trends Genet 29:403-411.

Gertz H, Fiehler K (2015) Human posterior parietal cortex encodes the movement goal in a pro-/anti-reach task. Journal of neurophysiology 114:170-183.

Grefkes C, Fink GR (2005) The functional organization of the intraparietal sulcus in humans and monkeys. J Anat 207:3-17.

Grosenick L, Marshel JH, Deisseroth K (2015) Closed-loop and activity-guided optogenetic control. Neuron 86:106-139. 
Gunaydin LA, Yizhar O, Berndt A, Sohal VS, Deisseroth K, Hegemann P (2010) Ultrafast optogenetic control. Nat Neurosci 13:387-392.

Han X, Boyden ES (2007) Multiple-color optical activation, silencing, and desynchronization of neural activity, with single-spike temporal resolution. PLoS One 2:e299.

Han X, Qian X, Bernstein JG, Zhou HH, Franzesi GT, Stern P, Bronson RT, Graybiel AM, Desimone R, Boyden ES (2009) Millisecond-timescale optical control of neural dynamics in the nonhuman primate brain. Neuron 62:191-198.

Han X, Chow BY, Zhou H, Klapoetke NC, Chuong A, Rajimehr R, Yang A, Baratta MV, Winkle J, Desimone R, Boyden ES (2011) A high-light sensitivity optical neural silencer: development and application to optogenetic control of nonhuman primate cortex. Front Syst Neurosci 5:18.

Harvey CD, Coen P, Tank DW (2012) Choice-specific sequences in parietal cortex during a virtual-navigation decision task. Nature 484:62-68.

Hawkins KM, Sayegh P, Yan X, Crawford JD, Sergio LE (2013) Neural activity in superior parietal cortex during rule-based visual-motor transformations. Journal of cognitive neuroscience 25:436-454.

Hennequin G, Vogels TP, Gerstner W (2014) Optimal control of transient dynamics in balanced networks supports generation of complex movements. Neuron 82:1394-1406.

Hommel B, Musseler J, Aschersleben G, Prinz W (2001) The Theory of Event Coding (TEC): a framework for perception and action planning. The Behavioral and brain sciences 24:849-878; discussion 878-937.

Hwang EJ, Hauschild M, Wilke M, Andersen RA (2012) Inactivation of the parietal reach region causes optic ataxia, impairing reaches but not saccades. Neuron 76:1021-1029.

Inoue K, Takada M, Matsumoto M (2015) Neuronal and behavioural modulations by pathway-selective optogenetic stimulation of the primate oculomotor system. Nat Commun 6:8378.

Jazayeri M, Lindbloom-Brown Z, Horwitz GD (2012) Saccadic eye movements evoked by optogenetic activation of primate V1. Nat Neurosci 15:1368-1370.

Jeannerod M (2001) Neural simulation of action: a unifying mechanism for motor cognition. NeuroImage 14:S103-109. 
Jennings JH, Rizzi G, Stamatakis AM, Ung RL, Stuber GD (2013) The inhibitory circuit architecture of the lateral hypothalamus orchestrates feeding. Science 341:1517-1521.

Johnson PB, Ferraina S, Bianchi L, Caminiti R (1996) Cortical networks for visual reaching: physiological and anatomical organization of frontal and parietal lobe arm regions. Cerebral cortex (New York, NY : 1991) 6:102-119.

Kalaska JF (1996) Parietal cortex area 5 and visuomotor behavior. CanJPhysiol Pharmacol 74:483-498.

Kalaska JF, Scott SH, Cisek P, Sergio LE (1997) Cortical control of reaching movements. Current opinion in neurobiology 7:849-859.

Kaufman MT, Churchland MM, Ryu SI, Shenoy KV (2014) Cortical activity in the null space: permitting preparation without movement. Nat Neurosci 17:440-448.

Klaes C, Westendorff S, Chakrabarti S, Gail A (2011) Choosing goals, not rules: deciding among rule-based action plans. Neuron 70:536-548.

Klaes C, Schneegans S, Schoner G, Gail A (2012) Sensorimotor learning biases choice behavior: a learning neural field model for decision making. PLoS Comput Biol 8:e1002774.

Kuang S, Morel P, Gail A (2016) Planning Movements in Visual and Physical Space in Monkey Posterior Parietal Cortex. Cerebral cortex (New York, NY : 1991) 26:731-747.

Kurata K (1989) Distribution of neurons with set- and movement-related activity before hand and foot movements in the premotor cortex of rhesus monkeys. Exp Brain Res 77:245-256.

Kurata K, Hoffman DS (1994) Differential effects of muscimol microinjection into dorsal and ventral aspects of the premotor cortex of monkeys. Journal of neurophysiology 71:1151-1164.

Lee JH, Durand R, Gradinaru V, Zhang F, Goshen I, Kim DS, Fenno LE, Ramakrishnan C, Deisseroth K (2010) Global and local fMRI signals driven by neurons defined optogenetically by type and wiring. Nature 465:788-792.

Lehmann SJ, Scherberger H (2013) Reach and gaze representations in macaque parietal and premotor grasp areas. The Journal of neuroscience : the official journal of the Society for Neuroscience 33:7038-7049.

Li X, Gutierrez DV, Hanson MG, Han J, Mark MD, Chiel H, Hegemann P, Landmesser LT, Herlitze S (2005) Fast noninvasive activation and inhibition of neural and network activity by vertebrate rhodopsin and green algae 
channelrhodopsin. Proceedings of the National Academy of Sciences of the United States of America 102:17816-17821.

Lu Y, Truccolo W, Wagner FB, Vargas-Irwin CE, Ozden I, Zimmermann JB, May T, Agha NS, Wang J, Nurmikko AV (2015) Optogenetically induced spatiotemporal gamma oscillations and neuronal spiking activity in primate motor cortex. Journal of neurophysiology 113:3574-3587.

Luppino G, Calzavara R, Rozzi S, Matelli M (2001) Projections from the superior temporal sulcus to the agranular frontal cortex in the macaque. Eur $\mathrm{J}$ Neurosci 14:1035-1040.

Luppino G, Rozzi S, Calzavara R, Matelli M (2003) Prefrontal and agranular cingulate projections to the dorsal premotor areas F2 and F7 in the macaque monkey. Eur J Neurosci 17:559-578.

Machens CK, Romo R, Brody CD (2010) Functional, but not anatomical, separation of "what" and "when" in prefrontal cortex. J Neurosci 30:350-360.

Mante V, Sussillo D, Shenoy KV, Newsome WT (2013) Context-dependent computation by recurrent dynamics in prefrontal cortex. Nature 503:78-84.

Marconi B, Genovesio A, Battaglia-Mayer A, Ferraina S, Squatrito S, Molinari M, Lacquaniti F, Caminiti R (2001) Eye-hand coordination during reaching. I. Anatomical relationships between parietal and frontal cortex. Cerebral cortex (New York, NY : 1991) 11:513-527.

Markov NT et al. (2014) A weighted and directed interareal connectivity matrix for macaque cerebral cortex. Cerebral cortex (New York, NY : 1991) 24:17-36.

Martinez-Vazquez P, Gail A (2018) Directed Interaction Between Monkey Premotor and Posterior Parietal Cortex During Motor-Goal Retrieval from Working Memory. Cerebral cortex (New York, NY : 1991) 28:1866-1881.

Matelli M, Govoni P, Galletti C, Kutz DF, Luppino G (1998) Superior area 6 afferents from the superior parietal lobule in the macaque monkey. J Comp Neurol 402:327-352.

Mattis J, Tye KM, Ferenczi EA, Ramakrishnan C, O'Shea DJ, Prakash R, Gunaydin LA, Hyun M, Fenno LE, Gradinaru V, Yizhar O, Deisseroth K (2011) Principles for applying optogenetic tools derived from direct comparative analysis of microbial opsins. Nat Methods 9:159-172.

McGuire LM, Sabes PN (2011) Heterogeneous representations in the superior parietal lobule are common across reaches to visual and proprioceptive targets. Journal of Neuroscience 31:6661-6673. 
Michaels JA, Dann B, Scherberger H (2016) Neural Population Dynamics during Reaching Are Better Explained by a Dynamical System than Representational Tuning. PLoS computational biology 12:e1005175.

Mulliken GH, Musallam S, Andersen RA (2008) Forward estimation of movement state in posterior parietal cortex. ProcNatlAcadSciUSA 105:8170-8177.

Nagel G, Brauner M, Liewald JF, Adeishvili N, Bamberg E, Gottschalk A (2005) Light activation of channelrhodopsin-2 in excitable cells of Caenorhabditis elegans triggers rapid behavioral responses. Curr Biol 15:2279-2284.

Nassi JJ, Avery MC, Cetin AH, Roe AW, Reynolds JH (2015) Optogenetic Activation of Normalization in Alert Macaque Visual Cortex. Neuron 86:15041517.

Ochiai T, Mushiake H, Tanji J (2002) Effects of image motion in the dorsal premotor cortex during planning of an arm movement. Journal of Neurophysiology 88:2167-2171.

Ochiai T, Mushiake H, Tanji J (2005) Involvement of the ventral premotor cortex in controlling image motion of the hand during performance of a target-capturing task. Cerebral Cortex 15:929-937.

Ohbayashi M, Picard N, Strick PL (2016) Inactivation of the Dorsal Premotor Area Disrupts Internally Generated, But Not Visually Guided, Sequential Movements. J Neurosci 36:1971-1976.

Passarelli L, Rosa MG, Gamberini M, Bakola S, Burman KJ, Fattori P, Galletti C (2011) Cortical connections of area V6Av in the macaque: a visual-input node to the eye/hand coordination system. J Neurosci 31:1790-1801.

Pesaran B, Nelson MJ, Andersen RA (2006) Dorsal premotor neurons encode the relative position of the hand, eye, and goal during reach planning. Neuron 51:125134.

Pesaran B, Nelson MJ, Andersen RA (2008) Free choice activates a decision circuit between frontal and parietal cortex. Nature 453:406-409.

Petreanu L, Mao T, Sternson SM, Svoboda K (2009) The subcellular organization of neocortical excitatory connections. Nature 457:1142-1145.

Petrides M, Pandya DN (1999) Dorsolateral prefrontal cortex: comparative cytoarchitectonic analysis in the human and the macaque brain and corticocortical connection patterns. Eur J Neurosci 11:1011-1036.

Petrides M, Pandya DN (2006) Efferent association pathways originating in the caudal prefrontal cortex in the macaque monkey. J Comp Neurol 498:227-251. 
Rajasethupathy P, Sankaran S, Marshel JH, Kim CK, Ferenczi E, Lee SY, Berndt A, Ramakrishnan C, Jaffe A, Lo M, Liston C, Deisseroth K (2015) Projections from neocortex mediate top-down control of memory retrieval. Nature 526:653659.

Raposo D, Kaufman MT, Churchland AK (2014) A category-free neural population supports evolving demands during decision-making. Nat Neurosci 17:1784-1792.

Ruiz O, Lustig BR, Nassi JJ, Cetin A, Reynolds JH, Albright TD, Callaway EM, Stoner GR, Roe AW (2013) Optogenetics through windows on the brain in the nonhuman primate. Journal of neurophysiology 110:1455-1467.

Sadtler PT, Quick KM, Golub MD, Chase SM, Ryu SI, Tyler-Kabara EC, Yu BM, Batista AP (2014) Neural constraints on learning. Nature 512:423-426.

Scherberger H, Andersen RA (2007) Target selection signals for arm reaching in the posterior parietal cortex. J Neurosci 27:2001-2012.

Schwartz AB, Moran DW, Reina GA (2004) Differential representation of perception and action in the frontal cortex. Science 303:380-383.

Scott SH (2004) Optimal feedback control and the neural basis of volitional motor control. Nature reviews Neuroscience 5:532-546.

Shadmehr R, Smith MA, Krakauer JW (2010) Error correction, sensory prediction, and adaptation in motor control. Annual Review of Neuroscience 33:89-108.

Shen L, Alexander GE (1997) Preferential representation of instructed target location versus limb trajectory in dorsal premotor area. Journal of Neurophysiology 77:1195-1212.

Shenoy KV, Sahani M, Churchland MM (2013) Cortical control of arm movements: a dynamical systems perspective. Annu Rev Neurosci 36:337-359.

Shin YK, Proctor RW, Capaldi EJ (2010) A review of contemporary ideomotor theory. Psychological bulletin 136:943-974.

Snyder LH, Batista AP, Andersen RA (1997) Coding of intention in the posterior parietal cortex. Nature 386:167-170.

Stauffer WR, Lak A, Yang A, Borel M, Paulsen O, Boyden ES, Schultz W (2016) Dopamine Neuron-Specific Optogenetic Stimulation in Rhesus Macaques. Cell 166:1564-1571 e1566.

Stetson C, Andersen RA (2014) The parietal reach region selectively antisynchronizes with dorsal premotor cortex during planning. J Neurosci 34:1194811958. 
Stopfer M, Jayaraman V, Laurent G (2003) Intensity versus identity coding in an olfactory system. Neuron 39:991-1004.

Stuber GD, Sparta DR, Stamatakis AM, van Leeuwen WA, Hardjoprajitno JE, Cho S, Tye KM, Kempadoo KA, Zhang F, Deisseroth K, Bonci A (2011) Excitatory transmission from the amygdala to nucleus accumbens facilitates reward seeking. Nature 475:377-380.

Sussillo D, Churchland MM, Kaufman MT, Shenoy KV (2015) A neural network that finds a naturalistic solution for the production of muscle activity. Nat Neurosci 18:1025-1033.

Tamura K, Takeda M, Setsuie R, Tsubota T, Hirabayashi T, Miyamoto K, Miyashita Y (2017) Conversion of object identity to object-general semantic value in the primate temporal cortex. Science 357:687-692.

Tanne-Gariepy J, Rouiller EM, Boussaoud D (2002) Parietal inputs to dorsal versus ventral premotor areas in the macaque monkey: evidence for largely segregated visuomotor pathways. Exp Brain Res 145:91-103.

Tye KM, Prakash R, Kim SY, Fenno LE, Grosenick L, Zarabi H, Thompson KR, Gradinaru V, Ramakrishnan C, Deisseroth K (2011) Amygdala circuitry mediating reversible and bidirectional control of anxiety. Nature 471:358-362.

Wallis JD, Miller EK (2003) From rule to response: neuronal processes in the premotor and prefrontal cortex. Journal of neurophysiology 90:1790-1806.

Wallis JD, Anderson KC, Miller EK (2001) Single neurons in prefrontal cortex encode abstract rules. Nature 411:953-956.

Wang J, Narain D, Hosseini EA, Jazayeri M (2018) Flexible timing by temporal scaling of cortical responses. Nat Neurosci 21:102-110.

Warden MR, Selimbeyoglu A, Mirzabekov JJ, Lo M, Thompson KR, Kim SY, Adhikari A, Tye KM, Frank LM, Deisseroth K (2012) A prefrontal cortexbrainstem neuronal projection that controls response to behavioural challenge. Nature 492:428-432.

Waszak F, Cardoso-Leite P, Hughes G (2012) Action effect anticipation: neurophysiological basis and functional consequences. Neuroscience and biobehavioral reviews 36:943-959.

Westendorff S, Klaes C, Gail A (2010) The cortical timeline for deciding on reach motor goals. J Neurosci 30:5426-5436. 
Wise SP, Boussaoud D, Johnson PB, Caminiti R (1997) Premotor and parietal cortex: corticocortical connectivity and combinatorial computations. Annual Review of Neuroscience 20:25-42.

Yuste R (2015) From the neuron doctrine to neural networks. Nat Rev Neurosci 16:487-497.

Zhang F, Wang LP, Brauner M, Liewald JF, Kay K, Watzke N, Wood PG, Bamberg E, Nagel G, Gottschalk A, Deisseroth K (2007) Multimodal fast optical interrogation of neural circuitry. Nature 446:633-639. 